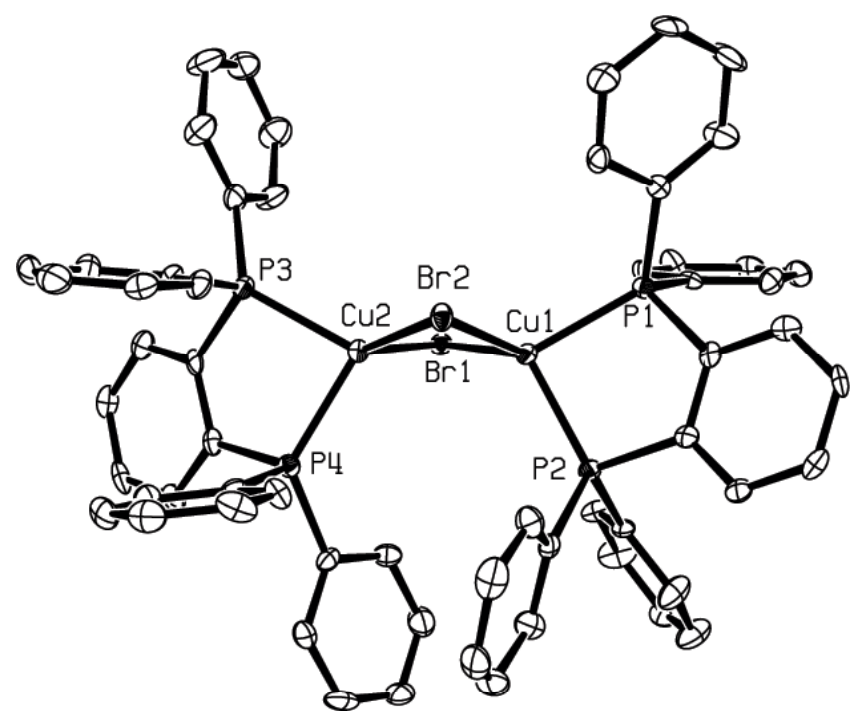

(A)

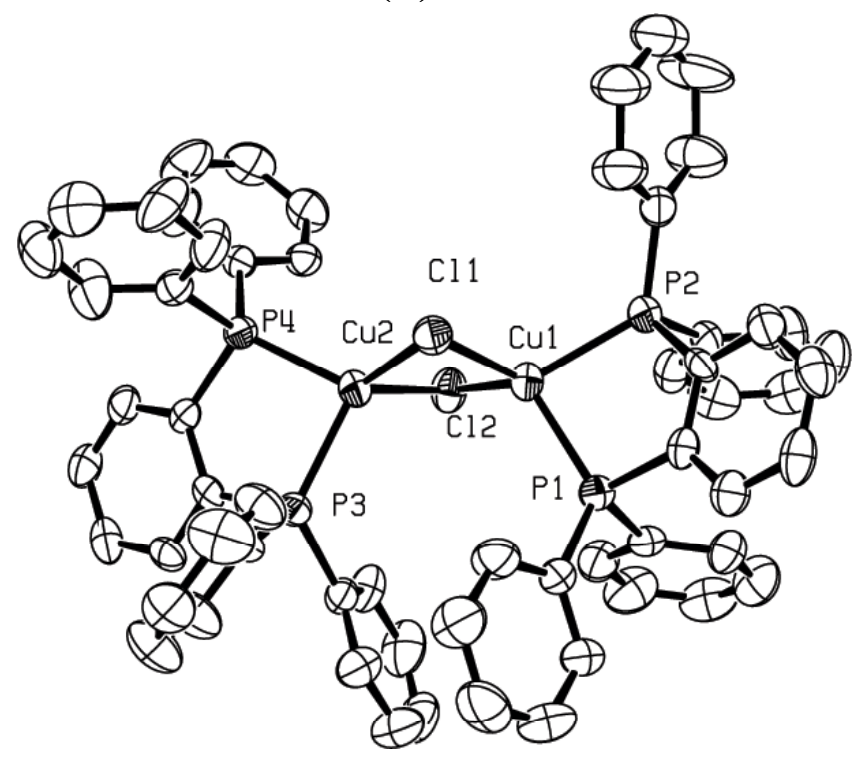

(B)

Figure S1. ORTEP diagram of (2) (A) and (3) (B). 

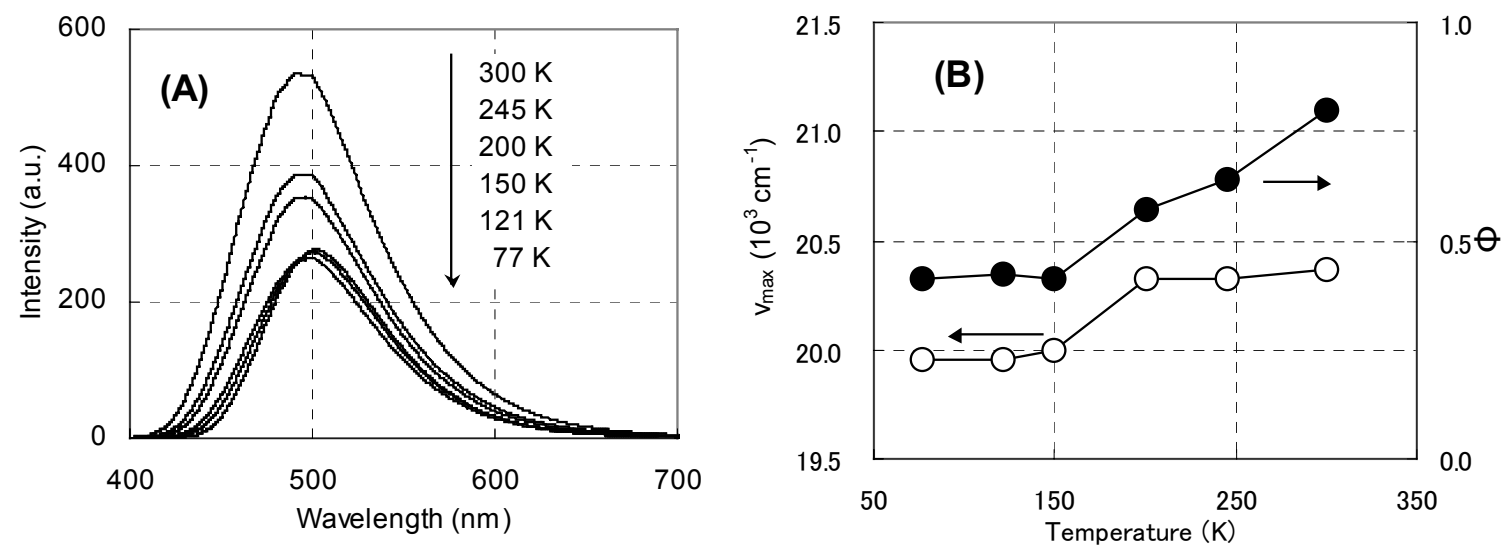

Figure S2. Temperature-dependent emission spectra of the complex (4) in the range of 77 $300 \mathrm{~K}$ in solid states (A) and plots of emission peak energy and quantum yield $(\Phi)$ vs temperature (B), obtained with the excitation wavelength at $350 \mathrm{~nm}$. 

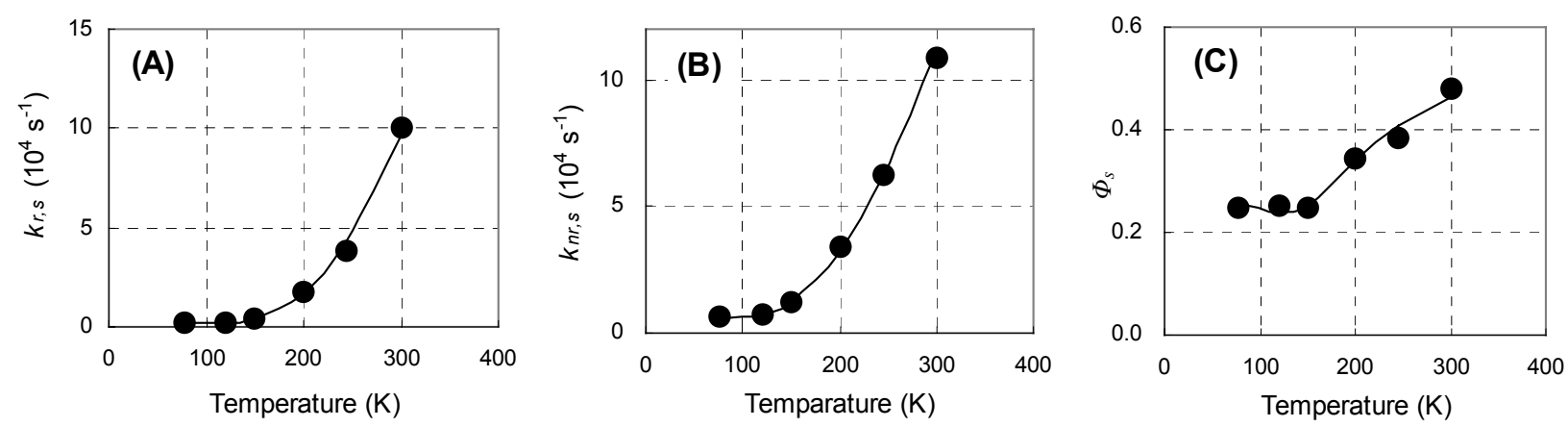

Figure S3. Radiative constant, $k_{r, s}$ (A), non-radiative constant, $k_{n r, s}(\mathbf{B})$, and emission quantum yield, $\Phi_{s}(\mathbf{C})$, for the slower decay component of the complex (4) in the solid state, represented as a function of $\mathrm{T}$. The closed circles are observed data at the temperatures and the solid lines are the calculated ones with the use of Equation (10)-(16) and parameters listed in Table 3. 

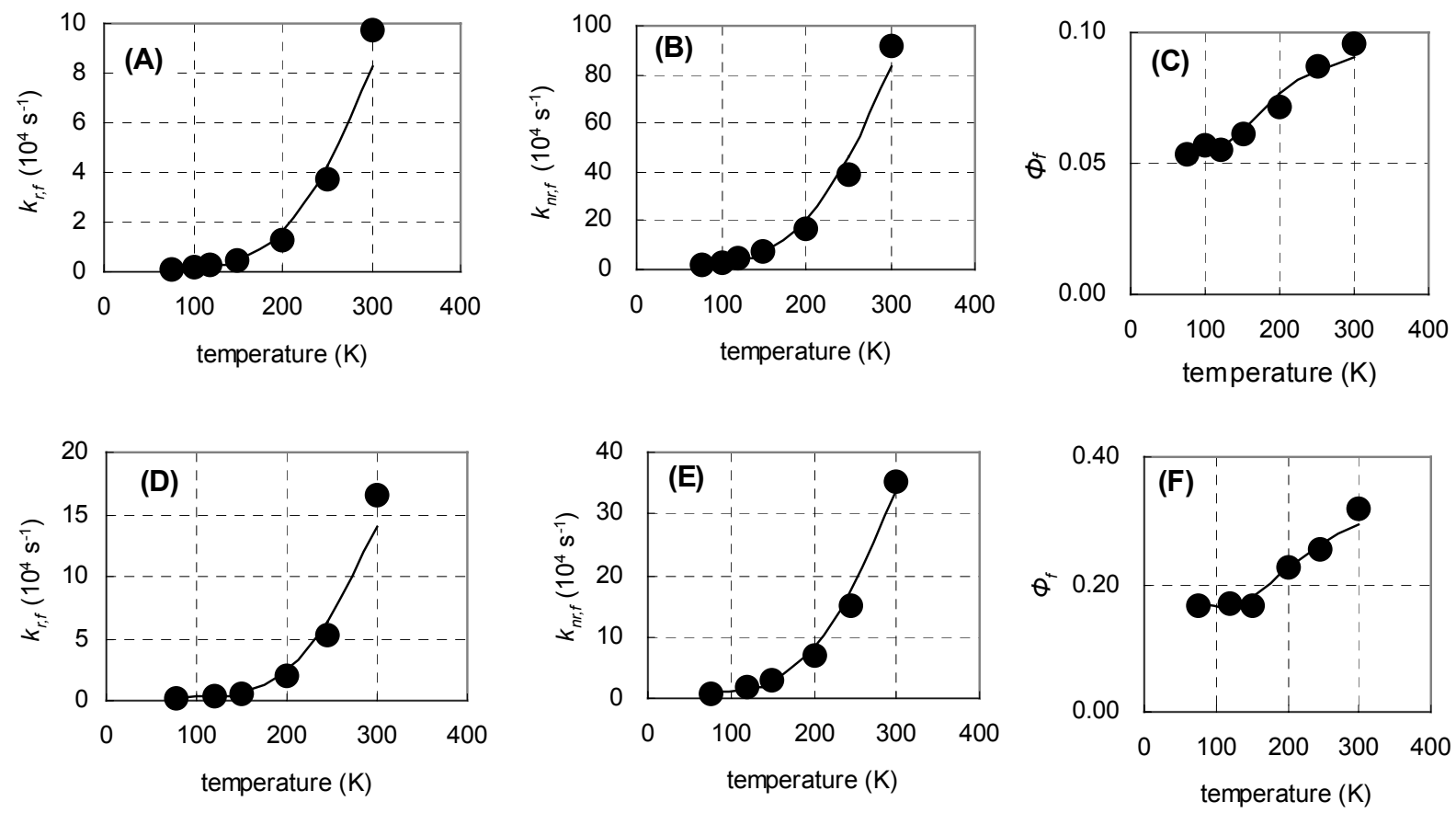

Figure S4. Radiative constant, $k_{r, f}\left[(\mathbf{A})\right.$ and (D)], non-radiative constant, $k_{n r, f}[(\mathbf{B})$ and (E)], and emission quantum yield, $\Phi_{f}[(\mathbf{C})$ and $(\mathbf{F})]$, for the faster decay component of the complex (1) and (4) in the solid state, represented as a function of T: (A), (B) and (C) are for the complex (1) and (C), (D) and (E) are for the complex (4). The closed circles are observed data at the temperatures and the solid lines are the calculated ones with the use of Equation (10)-(16) and parameters listed in Table S2. 

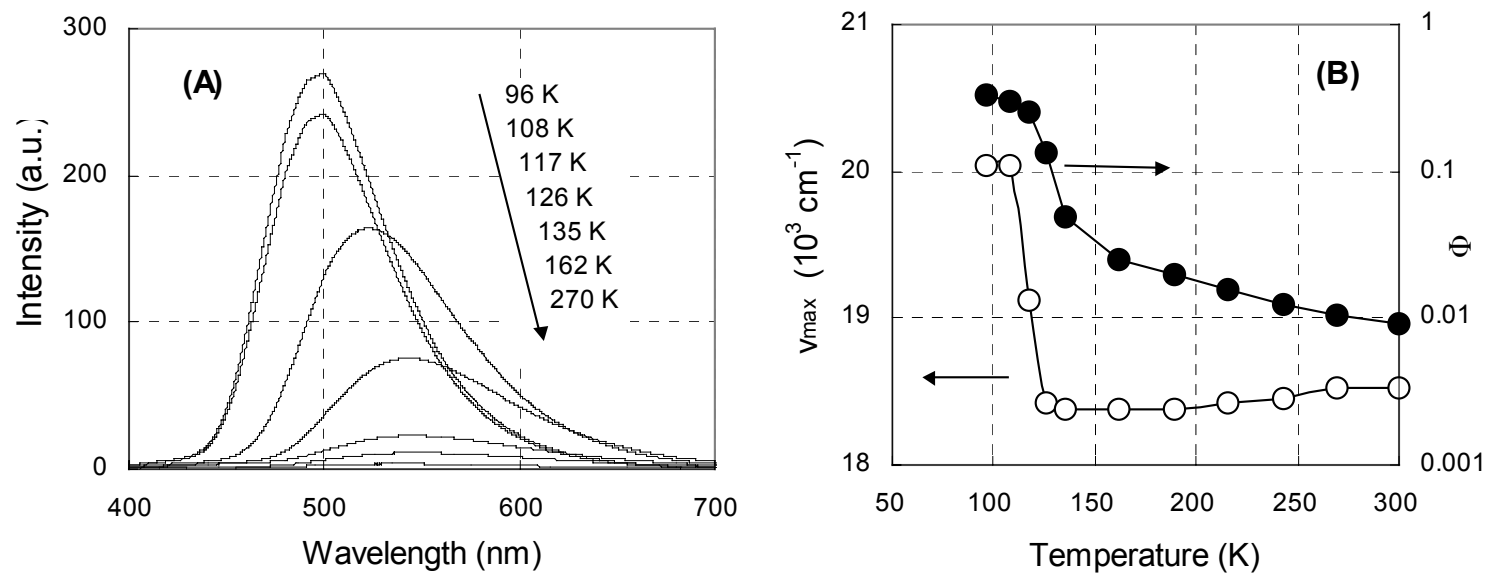

Figure S5. Temparature-dependent emission spectra of the complex (4) (A) in the range of 96-300 $\mathrm{K}$ in the $2 \mathrm{mTHF}$ solutions, and plots of emission peak energy $\left(v_{\max }\right)$ and quantum yield (Ф) vs temperature of (4) (B). 


$$
\mu
$$



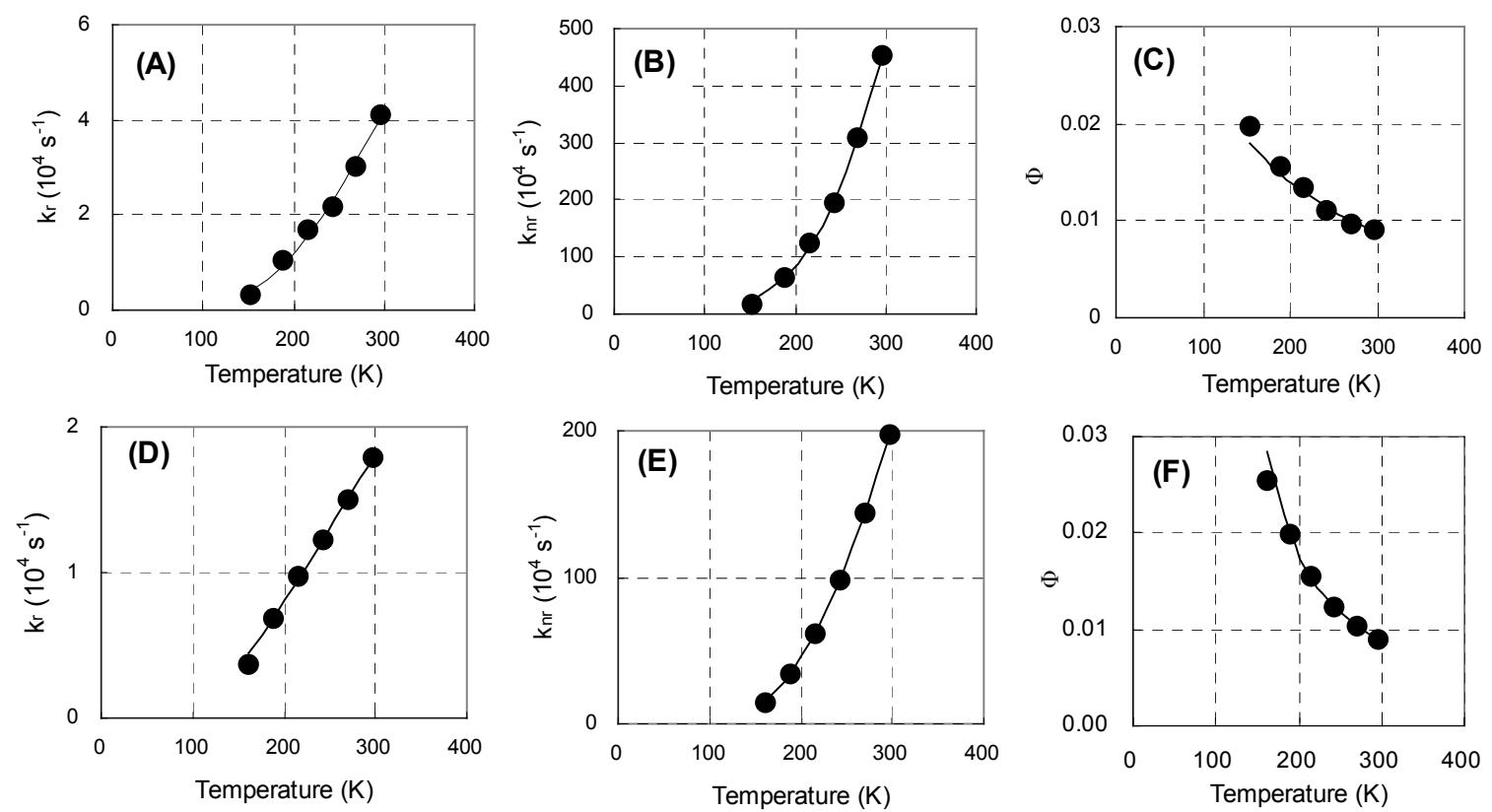

Figure S7. Radiative constant, $k_{r}\left[(\mathbf{A})\right.$ and (D)], non-radiative constant, $k_{n r}[(\mathbf{B})$ and (E)], and emission quantum yield, $\Phi[(\mathbf{C})$ and (F)], for the complex (1) and (4) in $2 \mathrm{mTHF}$ solutions, represented as a function of T: (A), (B) and (C) are for the complex (1) and (C), (D) and (E) are for the complex (4). The closed circles are observed data at the temperatures and the solid lines are the calculated ones with the use of Equation (10)-(16) and parameters listed in Table 4. 
Table S1. Selected Crystallographic Data for the Complex (1)-(4).

(1)

\begin{tabular}{lllll}
\hline \multicolumn{1}{c}{$(1)$} & \multicolumn{1}{c}{$(\mathbf{2})$} & \multicolumn{1}{c}{$(\mathbf{3})$} \\
formula & $\mathrm{C}_{60} \mathrm{H}_{48} \mathrm{Cu}_{2} \mathrm{I}_{2} \mathrm{P}_{4}$ & $\mathrm{C}_{60} \mathrm{H}_{48} \mathrm{Br}_{2} \mathrm{Cu}_{2} \mathrm{P}_{4}$ & $\mathrm{C}_{60} \mathrm{H}_{48} \mathrm{Cl}_{2} \mathrm{Cu}_{2} \mathrm{P}_{4}$ & $\mathrm{C}_{48} \mathrm{H}_{40} \mathrm{CuIP}_{3}$ \\
mol wt & 1273.84 & 1179.83 & 1090.93 & 900.22 \\
cryst syst & monoclinic & monoclinic & monoclinic & monoclinic \\
space group & $P 2_{1}(\mathrm{No} .4)$ & $P 2_{1}(\mathrm{No} .4)$ & $P 2_{1}(\mathrm{No} .4)$ & $P 2_{1}$ (No. 4) \\
$a, \AA$ & $11.61(1)$ & $11.20(1)$ & $11.137(8)$ & $15.11(1)$ \\
$b, \AA$ & $11.61(1)$ & $18.73(2)$ & $18.74(2)$ & $14.67(1)$ \\
$c, \AA$ & $24.36(2)$ & $12.12(1)$ & $12.303(8)$ & $18.33(2)$ \\
$\beta$, deg & $98.29(4)$ & $97.59(4)$ & $97.45(3)$ & $99.60(4)$ \\
$V, \AA^{3}$ & $5346(10)$ & $2520(4)$ & $2545(3)$ & $4004(5)$ \\
$Z$ & 4 & 2 & 2 & 4 \\
$D_{\text {calcd. }}$ g/cm & 1.583 & 1.554 & 1.423 & 1.493 \\
total no. of reflns & 46171 & 24210 & 23769 & 35524 \\
no. of unique reflns & 21811 & 24182 & 11026 & 35498 \\
$R$ & 0.0320 & 0.0450 & 0.042 & 0.037 \\
$R_{\mathrm{w}}$ & 0.0670 & 0.1090 & 0.052 & 0.082 \\
temp $\left({ }^{\circ} \mathrm{C}\right)$ & 23 & -173 & 20 & -173
\end{tabular}


Table S2. Kinetics parameters of the faster decay component for the complex (1) and (4) in solid state*.

\begin{tabular}{ccc}
\hline complex & $\mathbf{( 1 )}$ & $\mathbf{( 4 )}$ \\
\hline \hline$\Delta H_{f}$ & 2.0 & 2.2 \\
$k_{L, r, f}$ & $1.6 \times 10^{3}$ & $2.2 \times 10^{3}$ \\
$k_{H, r, f}$ & $1.0 \times 10^{8}$ & $1.0 \times 10^{8}$ \\
$\mathrm{~A}$ & 0.024 & 0.053 \\
$\Delta E_{L}$ & 1.8 & 1.8 \\
$\Delta E_{H}$ & 0 & 0 \\
$k_{L, n r, f}$ & $1.7 \times 10^{7}$ & $0.7 \times 10^{7}$ \\
$k_{L, n r, f}(0)$ & $2.7 \times 10^{4}$ & $1.1 \times 10^{4}$ \\
$k_{H, n, f}$ & 0 & 0 \\
$k_{H, n, f}(0)$ & 0 & 0 \\
\hline
\end{tabular}

${ }^{*}$ Energy separation, $\Delta E$ and $\Delta H$, are in $\mathrm{kcal} / \mathrm{mol}$ and rate constants are in $\mathrm{s}^{-1}$. 


\section{Table S3.}

\section{$[\mathrm{Cu}(\mu \mathrm{l}) \mathrm{dppb}]_{2}(\mathbf{1})$}

\section{EXPERIMENTAL DETAILS}

A. Crystal Data

Empirical Formula
Formula Weight
Crystal Color, Habit
Crystal Dimensions
Crystal System
Lattice Type
Indexing Images
Detector Position
Pixel Size
Lattice Parameters

Space Group

$Z$ value

$D_{\text {calc }}$

$\mathrm{F}_{000}$

$\mu(\mathrm{MoK} \alpha)$

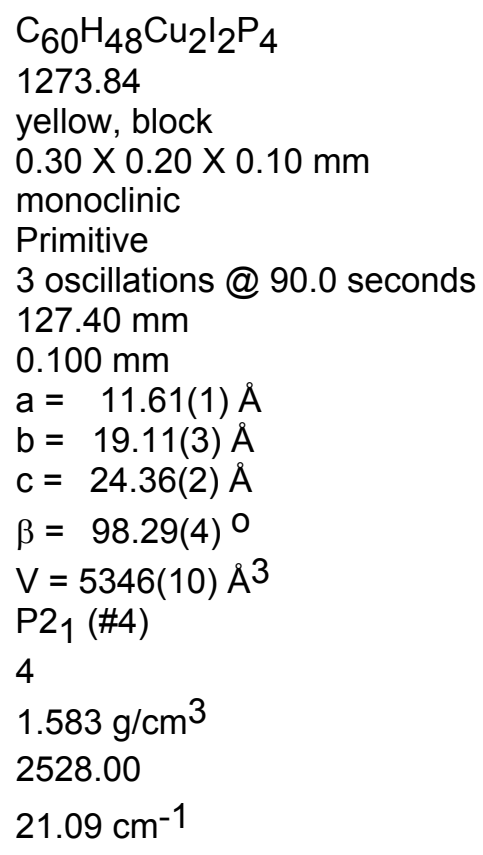

B. Intensity Measurements
Diffractometer

Radiation

Detector Aperture

Data Images

$\omega$ oscillation Range $(\chi=45.0, \phi=30.0)$

Exposure Rate

$\omega$ oscillation Range ( $\chi=45.0, \phi=180.0)$

Exposure Rate

Detector Position

Pixel Size

$2 \theta_{\max }$

No. of Reflections Measured

\author{
Rigaku RAXIS-RAPID \\ $\operatorname{MoK} \alpha(\lambda=0.71075 \AA)$ \\ graphite monochromated \\ $280 \mathrm{~mm} \times 256 \mathrm{~mm}$ \\ 73 exposures \\ $130.0-190.0^{\circ}$ \\ $40.2 \mathrm{sec} . / \mathrm{O}$ \\ $0.0-159.0^{\circ}$ \\ $40.2 \mathrm{sec} . / \mathrm{O}$ \\ $127.40 \mathrm{~mm}$ \\ $0.100 \mathrm{~mm}$ \\ $54.9^{\circ}$ \\ Total: 46171
}


Corrections

Unique: $21811\left(R_{\text {int }}=0.047\right)$

Lorentz-polarization

Absorption

(trans. factors: $0.5615-1.0000$ )

C. Structure Solution and Refinement

Structure Solution

Refinement

Function Minimized

Least Squares Weights

$2 \theta$ max cutoff

Anomalous Dispersion

No. Observations (All reflections)

No. Variables

Reflection/Parameter Ratio

Residuals: $R$ (All reflections)

Residuals: R1 (I>2.00 $\sigma(\mathrm{I}))$

Residuals: wR2 (All reflections)

Goodness of Fit Indicator

Max Shift/Error in Final Cycle

Maximum peak in Final Diff. Map

Minimum peak in Final Diff. Map
Patterson Methods (DIRDIF99 PATTY)

Full-matrix least-squares on $\mathrm{F}^{2}$

$\Sigma \mathrm{w}\left(\mathrm{Fo}^{2}-\mathrm{Fc}^{2}\right)^{2}$

$1 /\left[0.0001 \mathrm{Fo}^{2}+0.3000 \sigma\left(\mathrm{Fo}^{2}\right)\right] /\left(4 \mathrm{Fo}^{2}\right)$

$54.9^{\circ}$

All non-hydrogen atoms

21811

1321

16.51

0.075

0.032

0.067

1.004

0.000

$1.56 e^{-/ / \AA^{3}}$

$-1.41 e^{-} / \AA^{3}$

Table 4. Bond lengths $(\AA)$

$\begin{array}{llllll}\text { atom } & \text { atom } & \text { distance } & \text { atom } & \text { atom } & \text { distance } \\ \mathrm{I}(1) & \mathrm{Cu}(1) & 2.6155(8) & \mathrm{I}(1) & \mathrm{Cu}(2) & 2.6528(8) \\ \mathrm{I}(2) & \mathrm{Cu}(1) & 2.6729(9) & \mathrm{I}(2) & \mathrm{Cu}(2) & 2.5960(8) \\ \mathrm{I}(3) & \mathrm{Cu}(3) & 2.6198(6) & \mathrm{I}(3) & \mathrm{Cu}(4) & 2.6587(8) \\ \mathrm{I}(4) & \mathrm{Cu}(3) & 2.6806(8) & \mathrm{I}(4) & \mathrm{Cu}(4) & 2.5787(9) \\ \mathrm{Cu}(1) & \mathrm{P}(1) & 2.283(2) & \mathrm{Cu}(1) & \mathrm{P}(2) & 2.274(2) \\ \mathrm{Cu}(2) & \mathrm{P}(3) & 2.289(2) & \mathrm{Cu}(2) & \mathrm{P}(4) & 2.279(2) \\ \mathrm{Cu}(3) & \mathrm{P}(5) & 2.286(2) & \mathrm{Cu}(3) & \mathrm{P}(6) & 2.272(2) \\ \mathrm{Cu}(4) & \mathrm{P}(7) & 2.282(2) & \mathrm{Cu}(4) & \mathrm{P}(8) & 2.272(2) \\ \mathrm{P}(1) & \mathrm{C}(6) & 1.836(6) & \mathrm{P}(1) & \mathrm{C}(7) & 1.826(6) \\ \mathrm{P}(1) & \mathrm{C}(13) & 1.824(7) & \mathrm{P}(2) & \mathrm{C}(1) & 1.852(6) \\ \mathrm{P}(2) & \mathrm{C}(19) & 1.810(6) & \mathrm{P}(2) & \mathrm{C}(25) & 1.816(7) \\ \mathrm{P}(3) & \mathrm{C}(31) & 1.827(7) & \mathrm{P}(3) & \mathrm{C}(37) & 1.795(8) \\ \mathrm{P}(3) & \mathrm{C}(43) & 1.832(7) & \mathrm{P}(4) & \mathrm{C}(36) & 1.830(6) \\ \mathrm{P}(4) & \mathrm{C}(49) & 1.809(7) & \mathrm{P}(4) & \mathrm{C}(55) & 1.815(7) \\ \mathrm{P}(5) & \mathrm{C}(61) & 1.827(6) & \mathrm{P}(5) & \mathrm{C}(67) & 1.819(6) \\ \mathrm{P}(5) & \mathrm{C}(73) & 1.820(6) & \mathrm{P}(6) & \mathrm{C}(66) & 1.847(5) \\ \mathrm{P}(6) & \mathrm{C}(79) & 1.806(6) & \mathrm{P}(6) & \mathrm{C}(85) & 1.831(7) \\ \mathrm{P}(7) & \mathrm{C}(91) & 1.830(6) & \mathrm{P}(7) & \mathrm{C}(97) & 1.812(7) \\ \mathrm{P}(7) & \mathrm{C}(103) & 1.810(6) & \mathrm{P}(8) & \mathrm{C}(96) & 1.839(6) \\ \mathrm{P}(8) & \mathrm{C}(109) & 1.800(6) & \mathrm{P}(8) & \mathrm{C}(115) & 1.813(7) \\ \mathrm{C}(1) & \mathrm{C}(2) & 1.389(9) & \mathrm{C}(1) & \mathrm{C}(6) & 1.395(9) \\ \mathrm{C}(2) & \mathrm{C}(3) & 1.390(9) & \mathrm{C}(3) & \mathrm{C}(4) & 1.37(1)\end{array}$




$\begin{array}{llllll}\mathrm{C}(4) & \mathrm{C}(5) & 1.36(1) & \mathrm{C}(5) & \mathrm{C}(6) & 1.400(8) \\ \mathrm{C}(7) & \mathrm{C}(8) & 1.35(1) & \mathrm{C}(7) & \mathrm{C}(12) & 1.338(9) \\ \mathrm{C}(8) & \mathrm{C}(9) & 1.39(1) & \mathrm{C}(9) & \mathrm{C}(10) & 1.37(1) \\ \mathrm{C}(10) & \mathrm{C}(11) & 1.32(1) & \mathrm{C}(11) & \mathrm{C}(12) & 1.38(1) \\ \mathrm{C}(13) & \mathrm{C}(14) & 1.382(8) & \mathrm{C}(13) & \mathrm{C}(18) & 1.381(9) \\ \mathrm{C}(14) & \mathrm{C}(15) & 1.38(1) & \mathrm{C}(15) & \mathrm{C}(16) & 1.35(1) \\ \mathrm{C}(16) & \mathrm{C}(17) & 1.37(1) & \mathrm{C}(17) & \mathrm{C}(18) & 1.36(1) \\ \mathrm{C}(19) & \mathrm{C}(20) & 1.34(1) & \mathrm{C}(19) & \mathrm{C}(24) & 1.37(1) \\ \mathrm{C}(20) & \mathrm{C}(21) & 1.39(1) & \mathrm{C}(21) & \mathrm{C}(22) & 1.33(1) \\ \mathrm{C}(22) & \mathrm{C}(23) & 1.35(1) & \mathrm{C}(23) & \mathrm{C}(24) & 1.36(1) \\ \mathrm{C}(25) & \mathrm{C}(26) & 1.37(1) & \mathrm{C}(25) & \mathrm{C}(30) & 1.40(1) \\ \mathrm{C}(26) & \mathrm{C}(27) & 1.39(1) & \mathrm{C}(27) & \mathrm{C}(28) & 1.38(1) \\ \mathrm{C}(28) & \mathrm{C}(29) & 1.34(1) & \mathrm{C}(29) & \mathrm{C}(30) & 1.36(1) \\ \mathrm{C}(31) & \mathrm{C}(32) & 1.380(8) & \mathrm{C}(31) & \mathrm{C}(36) & 1.40(1) \\ \mathrm{C}(32) & \mathrm{C}(33) & 1.388(9) & \mathrm{C}(33) & \mathrm{C}(34) & 1.38(1)\end{array}$

Table 4. Bond lengths $(\AA) \quad$-- continued

$\begin{array}{llllll}\text { atom } & \text { atom } & \text { distance } & \text { atom } & \text { atom } & \text { distance } \\ \mathrm{C}(34) & \mathrm{C}(35) & 1.393(9) & \mathrm{C}(35) & \mathrm{C}(36) & 1.392(9) \\ \mathrm{C}(37) & \mathrm{C}(38) & 1.38(1) & \mathrm{C}(37) & \mathrm{C}(42) & 1.39(1) \\ \mathrm{C}(38) & \mathrm{C}(39) & 1.39(2) & \mathrm{C}(39) & \mathrm{C}(40) & 1.33(2) \\ \mathrm{C}(40) & \mathrm{C}(41) & 1.36(2) & \mathrm{C}(41) & \mathrm{C}(42) & 1.37(1) \\ \mathrm{C}(43) & \mathrm{C}(44) & 1.35(1) & \mathrm{C}(43) & \mathrm{C}(48) & 1.400(9) \\ \mathrm{C}(44) & \mathrm{C}(45) & 1.38(1) & \mathrm{C}(45) & \mathrm{C}(46) & 1.38(1) \\ \mathrm{C}(46) & \mathrm{C}(47) & 1.36(1) & \mathrm{C}(47) & \mathrm{C}(48) & 1.37(1) \\ \mathrm{C}(49) & \mathrm{C}(50) & 1.38(1) & \mathrm{C}(49) & \mathrm{C}(54) & 1.39(1) \\ \mathrm{C}(50) & \mathrm{C}(51) & 1.36(1) & \mathrm{C}(51) & \mathrm{C}(52) & 1.36(2) \\ \mathrm{C}(52) & \mathrm{C}(53) & 1.35(2) & \mathrm{C}(53) & \mathrm{C}(54) & 1.37(1) \\ \mathrm{C}(55) & \mathrm{C}(56) & 1.382(9) & \mathrm{C}(55) & \mathrm{C}(60) & 1.38(1) \\ \mathrm{C}(56) & \mathrm{C}(57) & 1.38(1) & \mathrm{C}(57) & \mathrm{C}(58) & 1.34(1) \\ \mathrm{C}(58) & \mathrm{C}(59) & 1.36(1) & \mathrm{C}(59) & \mathrm{C}(60) & 1.39(1) \\ \mathrm{C}(61) & \mathrm{C}(62) & 1.405(8) & \mathrm{C}(61) & \mathrm{C}(66) & 1.400(8) \\ \mathrm{C}(62) & \mathrm{C}(63) & 1.38(1) & \mathrm{C}(63) & \mathrm{C}(64) & 1.38(1) \\ \mathrm{C}(64) & \mathrm{C}(65) & 1.375(8) & \mathrm{C}(65) & \mathrm{C}(66) & 1.399(8) \\ \mathrm{C}(67) & \mathrm{C}(68) & 1.389(8) & \mathrm{C}(67) & \mathrm{C}(72) & 1.360(9) \\ \mathrm{C}(68) & \mathrm{C}(69) & 1.378(9) & \mathrm{C}(69) & \mathrm{C}(70) & 1.37(1) \\ \mathrm{C}(70) & \mathrm{C}(71) & 1.35(1) & \mathrm{C}(71) & \mathrm{C}(72) & 1.40(1) \\ \mathrm{C}(73) & \mathrm{C}(74) & 1.35(1) & \mathrm{C}(73) & \mathrm{C}(78) & 1.35(1) \\ \mathrm{C}(74) & \mathrm{C}(75) & 1.38(1) & \mathrm{C}(75) & \mathrm{C}(76) & 1.35(2) \\ \mathrm{C}(76) & \mathrm{C}(77) & 1.33(1) & \mathrm{C}(77) & \mathrm{C}(78) & 1.37(1) \\ \mathrm{C}(79) & \mathrm{C}(80) & 1.381(9) & \mathrm{C}(79) & \mathrm{C}(84) & 1.37(1) \\ \mathrm{C}(80) & \mathrm{C}(81) & 1.38(1) & \mathrm{C}(81) & \mathrm{C}(82) & 1.35(1) \\ \mathrm{C}(82) & \mathrm{C}(83) & 1.34(1) & \mathrm{C}(83) & \mathrm{C}(84) & 1.37(1) \\ \mathrm{C}(85) & \mathrm{C}(86) & 1.39(1) & \mathrm{C}(85) & \mathrm{C}(90) & 1.39(1) \\ \mathrm{C}(86) & \mathrm{C}(87) & 1.39(1) & \mathrm{C}(87) & \mathrm{C}(88) & 1.37(1) \\ \mathrm{C}(88) & \mathrm{C}(89) & 1.38(1) & \mathrm{C}(89) & \mathrm{C}(90) & 1.37(1) \\ \mathrm{C}(91) & \mathrm{C}(92) & 1.390(8) & \mathrm{C}(91) & \mathrm{C}(96) & 1.407(9) \\ \mathrm{C}(92) & \mathrm{C}(93) & 1.38(1) & \mathrm{C}(93) & \mathrm{C}(94) & 1.37(1) \\ \mathrm{C}(94) & \mathrm{C}(95) & 1.373(9) & \mathrm{C}(95) & \mathrm{C}(96) & 1.382(9) \\ \mathrm{C}(97) & \mathrm{C}(98) & 1.38(1) & \mathrm{C}(97) & \mathrm{C}(102) & 1.40(1) \\ & & & & \end{array}$




\begin{tabular}{llllll}
$\mathrm{C}(98)$ & $\mathrm{C}(99)$ & $1.37(1)$ & $\mathrm{C}(99)$ & $\mathrm{C}(100)$ & $1.36(1)$ \\
$\mathrm{C}(100)$ & $\mathrm{C}(101)$ & $1.37(1)$ & $\mathrm{C}(101)$ & $\mathrm{C}(102)$ & $1.37(1)$ \\
$\mathrm{C}(103)$ & $\mathrm{C}(104)$ & $1.387(9)$ & $\mathrm{C}(103)$ & $\mathrm{C}(108)$ & $1.365(9)$ \\
$\mathrm{C}(104)$ & $\mathrm{C}(105)$ & $1.38(1)$ & $\mathrm{C}(105)$ & $\mathrm{C}(106)$ & $1.34(1)$ \\
$\mathrm{C}(106)$ & $\mathrm{C}(107)$ & $1.36(1)$ & $\mathrm{C}(107)$ & $\mathrm{C}(108)$ & $1.40(1)$ \\
\multicolumn{7}{c}{} & & & \\
\multicolumn{7}{r}{ Table 4. Bond lengths $(\AA)$} & - - continued & & & \\
atom & atom & distance & atom & atom & distance \\
$\mathrm{C}(109)$ & $\mathrm{C}(110)$ & $1.378(8)$ & $\mathrm{C}(109)$ & $\mathrm{C}(114)$ & $1.38(1)$ \\
$\mathrm{C}(110)$ & $\mathrm{C}(111)$ & $1.39(1)$ & $\mathrm{C}(111)$ & $\mathrm{C}(112)$ & $1.34(1)$ \\
$\mathrm{C}(112)$ & $\mathrm{C}(113)$ & $1.35(1)$ & $\mathrm{C}(113)$ & $\mathrm{C}(114)$ & $1.39(1)$ \\
$\mathrm{C}(115)$ & $\mathrm{C}(116)$ & $1.37(1)$ & $\mathrm{C}(115)$ & $\mathrm{C}(120)$ & $1.371(9)$ \\
$\mathrm{C}(116)$ & $\mathrm{C}(117)$ & $1.37(1)$ & $\mathrm{C}(117)$ & $\mathrm{C}(118)$ & $1.36(1)$ \\
$\mathrm{C}(118)$ & $\mathrm{C}(119)$ & $1.35(1)$ & $\mathrm{C}(119)$ & $\mathrm{C}(120)$ & $1.38(1)$
\end{tabular}

Table 6. Bond angles $\left(^{\circ}\right)$

\begin{tabular}{|c|c|c|c|c|c|c|c|}
\hline atom & atom & atom & angle & atom & atom & atom & angle \\
\hline $\mathrm{Cu}(2)$ & $\mathrm{I}(1)$ & $\mathrm{Cu}(1)$ & $66.74(2)$ & $\mathrm{I}(1)$ & $\mathrm{Cu}(1)$ & $\mathrm{I}(2)$ & $108.69(3)$ \\
\hline $\mathrm{I}(1)$ & $\mathrm{Cu}(1)$ & $P(1)$ & $117.70(5)$ & $\mathrm{I}(1)$ & $\mathrm{Cu}(1)$ & $P(2)$ & $123.56(6)$ \\
\hline I(1) & $\mathrm{Cu}(2)$ & $\mathrm{I}(2)$ & 109.91(3) & $\mathrm{I}(1)$ & $\mathrm{Cu}(2)$ & $P(3)$ & $107.62(6)$ \\
\hline $\mathrm{I}(1)$ & $\mathrm{Cu}(2)$ & $P(4)$ & $111.76(6)$ & $\mathrm{Cu}(2)$ & $\mathrm{I}(2)$ & $\mathrm{Cu}(1)$ & $66.71(2)$ \\
\hline I(2) & $\mathrm{Cu}(1)$ & $P(1)$ & $109.55(5)$ & $\mathrm{I}(2)$ & $\mathrm{Cu}(1)$ & $P(2)$ & $107.08(5)$ \\
\hline I(2) & $\mathrm{Cu}(2)$ & $P(3)$ & $121.09(6)$ & I(2) & $\mathrm{Cu}(2)$ & $P(4)$ & $117.98(5)$ \\
\hline $\mathrm{Cu}(4)$ & $\mathrm{I}(3)$ & $\mathrm{Cu}(3)$ & $65.66(2)$ & I(3) & $\mathrm{Cu}(3)$ & $\mathrm{I}(4)$ & $109.56(3)$ \\
\hline$I(3)$ & $\mathrm{Cu}(3)$ & $P(5)$ & $121.11(5)$ & $I(3)$ & $\mathrm{Cu}(3)$ & $P(6)$ & $119.86(5)$ \\
\hline I(3) & $\mathrm{Cu}(4)$ & $\mathrm{I}(4)$ & 111.54(3) & I(3) & $\mathrm{Cu}(4)$ & $P(7)$ & $104.13(5)$ \\
\hline I(3) & $\mathrm{Cu}(4)$ & $\mathrm{P}(8)$ & $110.38(6)$ & $\mathrm{Cu}(4)$ & $I(4)$ & $\mathrm{Cu}(3)$ & $65.90(2)$ \\
\hline I(4) & $\mathrm{Cu}(3)$ & $P(5)$ & $105.46(5)$ & $\mathrm{I}(4)$ & $\mathrm{Cu}(3)$ & $P(6)$ & $109.79(5)$ \\
\hline I(4) & $\mathrm{Cu}(4)$ & $P(7)$ & $118.91(6)$ & I(4) & $\mathrm{Cu}(4)$ & $P(8)$ & $121.92(6)$ \\
\hline$P(2)$ & $\mathrm{Cu}(1)$ & $P(1)$ & $88.46(6)$ & $\mathrm{Cu}(1)$ & $P(1)$ & $C(6)$ & $102.4(2)$ \\
\hline $\mathrm{Cu}(1)$ & $P(1)$ & $C(7)$ & $114.6(2)$ & $\mathrm{Cu}(1)$ & $P(1)$ & $C(13)$ & $125.1(2)$ \\
\hline $\mathrm{Cu}(1)$ & $P(2)$ & $C(1)$ & $102.2(2)$ & $\mathrm{Cu}(1)$ & $P(2)$ & $C(19)$ & $118.8(2)$ \\
\hline $\mathrm{Cu}(1)$ & $P(2)$ & $C(25)$ & $121.5(2)$ & $P(4)$ & $\mathrm{Cu}(2)$ & $P(3)$ & $86.47(6)$ \\
\hline $\mathrm{Cu}(2)$ & $P(3)$ & $C(31)$ & $100.5(2)$ & $\mathrm{Cu}(2)$ & $P(3)$ & $C(43)$ & $116.9(2)$ \\
\hline $\mathrm{Cu}(2)$ & $P(3)$ & $C(37)$ & $123.6(2)$ & $\mathrm{Cu}(2)$ & $P(4)$ & $C(36)$ & $101.0(2)$ \\
\hline $\mathrm{Cu}(2)$ & $P(4)$ & $C(55)$ & $113.7(2)$ & $\mathrm{Cu}(2)$ & $P(4)$ & $C(49)$ & $125.5(2)$ \\
\hline$P(6)$ & $\mathrm{Cu}(3)$ & $P(5)$ & $88.96(6)$ & $\mathrm{Cu}(3)$ & $P(5)$ & $C(61)$ & $102.4(2)$ \\
\hline $\mathrm{Cu}(3)$ & $P(5)$ & $C(67)$ & $116.0(2)$ & $\mathrm{Cu}(3)$ & $P(5)$ & $C(73)$ & $124.5(2)$ \\
\hline $\mathrm{Cu}(3)$ & $P(6)$ & $C(66)$ & $102.5(2)$ & $\mathrm{Cu}(3)$ & $P(6)$ & $C(79)$ & $120.1(2)$ \\
\hline $\mathrm{Cu}(3)$ & $P(6)$ & $C(85)$ & $119.6(2)$ & $P(8)$ & $\mathrm{Cu}(4)$ & $P(7)$ & $86.68(6)$ \\
\hline $\mathrm{Cu}(4)$ & $P(7)$ & $C(91)$ & $101.1(2)$ & $\mathrm{Cu}(4)$ & $P(7)$ & $C(103)$ & $116.4(2)$ \\
\hline $\mathrm{Cu}(4)$ & $P(7)$ & $C(97)$ & 124.3(2) & $\mathrm{Cu}(4)$ & $P(8)$ & $C(96)$ & $101.3(2)$ \\
\hline $\mathrm{Cu}(4)$ & $P(8)$ & $C(109)$ & $115.4(2)$ & $\mathrm{Cu}(4)$ & $P(8)$ & $C(115)$ & $125.5(2)$ \\
\hline$P(1)$ & $C(6)$ & $C(1)$ & $118.9(4)$ & $C(7)$ & $P(1)$ & $C(6)$ & 105.2(3) \\
\hline$P(1)$ & $C(6)$ & C(5) & $122.9(5)$ & $C(13)$ & $P(1)$ & $C(6)$ & $104.4(3)$ \\
\hline$P(1)$ & $C(7)$ & $C(8)$ & $123.5(5)$ & $P(1)$ & $C(7)$ & $C(12)$ & $118.5(4)$ \\
\hline$C(13)$ & $P(1)$ & $C(7)$ & $103.2(3)$ & $P(1)$ & $C(13)$ & $C(14)$ & $125.0(5)$ \\
\hline$P(1)$ & $C(13)$ & C(18) & $117.6(4)$ & $P(2)$ & $C(1)$ & $C(6)$ & $118.9(4)$ \\
\hline
\end{tabular}




$\begin{array}{llllllll}\mathrm{C}(19) & \mathrm{P}(2) & \mathrm{C}(1) & 102.6(3) & \mathrm{C}(25) & \mathrm{P}(2) & \mathrm{C}(1) & 103.6(3) \\ \mathrm{P}(2) & \mathrm{C}(1) & \mathrm{C}(2) & 121.3(5) & \mathrm{C}(25) & \mathrm{P}(2) & \mathrm{C}(19) & 105.3(3) \\ \mathrm{P}(2) & \mathrm{C}(19) & \mathrm{C}(20) & 120.3(5) & \mathrm{P}(2) & \mathrm{C}(19) & \mathrm{C}(24) & 123.1(6) \\ \mathrm{P}(2) & \mathrm{C}(25) & \mathrm{C}(26) & 120.3(5) & \mathrm{P}(2) & \mathrm{C}(25) & \mathrm{C}(30) & 121.7(5) \\ \mathrm{P}(3) & \mathrm{C}(31) & \mathrm{C}(36) & 117.7(4) & \mathrm{C}(43) & \mathrm{P}(3) & \mathrm{C}(31) & 102.3(3) \\ \mathrm{P}(3) & \mathrm{C}(31) & \mathrm{C}(32) & 123.6(6) & \mathrm{C}(37) & \mathrm{P}(3) & \mathrm{C}(31) & 104.8(3)\end{array}$

Table 6. Bond angles $\left(^{\circ}\right)$-- continued

\begin{tabular}{|c|c|c|c|c|c|c|c|}
\hline atom & atom & atom & angle & atom & atom & atom & angle \\
\hline$C(43)$ & $P(3)$ & $C(37)$ & $105.7(4)$ & $P(3)$ & $C(37)$ & $\mathrm{C}(38)$ & $123.3(7)$ \\
\hline$P(3)$ & $C(37)$ & $C(42)$ & $120.1(6)$ & $P(3)$ & $C(43)$ & C(44) & $119.7(5)$ \\
\hline$P(3)$ & $C(43)$ & $C(48)$ & $122.6(6)$ & $P(4)$ & $C(36)$ & $C(31)$ & $118.8(4)$ \\
\hline$C(55)$ & $P(4)$ & $C(36)$ & $104.4(3)$ & $P(4)$ & $C(36)$ & $C(35)$ & $121.0(5)$ \\
\hline C(49) & $P(4)$ & $C(36)$ & $104.5(3)$ & C(55) & $P(4)$ & $C(49)$ & $105.3(3)$ \\
\hline$P(4)$ & $C(49)$ & $C(50)$ & $126.6(6)$ & $P(4)$ & $C(49)$ & $C(54)$ & $117.4(6)$ \\
\hline$P(4)$ & $C(55)$ & $C(56)$ & $124.2(5)$ & $P(4)$ & $C(55)$ & $C(60)$ & $118.1(5)$ \\
\hline$P(5)$ & $C(61)$ & $C(66)$ & $119.4(4)$ & $C(67)$ & $P(5)$ & $C(61)$ & $104.5(3)$ \\
\hline$P(5)$ & $C(61)$ & $C(62)$ & $122.0(5)$ & $C(73)$ & $P(5)$ & $C(61)$ & $103.8(3)$ \\
\hline$P(5)$ & $C(67)$ & $C(68)$ & $124.2(5)$ & $P(5)$ & $C(67)$ & $C(72)$ & $118.1(4)$ \\
\hline$C(73)$ & $P(5)$ & $C(67)$ & $103.3(3)$ & $P(5)$ & $C(73)$ & $C(74)$ & $124.7(6)$ \\
\hline$P(5)$ & $C(73)$ & $C(78)$ & $119.8(5)$ & $P(6)$ & $C(66)$ & $C(61)$ & 119.1(4) \\
\hline $\mathrm{C}(79)$ & $P(6)$ & $C(66)$ & 103.2(3) & $C(85)$ & $\mathrm{P}(6)$ & $C(66)$ & $104.5(3)$ \\
\hline$P(6)$ & $C(66)$ & $C(65)$ & $121.6(4)$ & $C(85)$ & $P(6)$ & $C(79)$ & 104.5(3) \\
\hline$P(6)$ & $C(79)$ & $C(80)$ & $124.6(5)$ & $P(6)$ & $C(79)$ & $C(84)$ & $120.3(5)$ \\
\hline$P(6)$ & $C(85)$ & $C(86)$ & $117.5(5)$ & $P(6)$ & $C(85)$ & $C(90)$ & $123.6(5)$ \\
\hline$P(7)$ & $\mathrm{C}(91)$ & $C(96)$ & $117.9(4)$ & $C(103)$ & $P(7)$ & $\mathrm{C}(91)$ & 101.7(3) \\
\hline$P(7)$ & $C(91)$ & $C(92)$ & $123.5(5)$ & $\mathrm{C}(97)$ & $P(7)$ & $C(91)$ & $105.8(3)$ \\
\hline$C(103)$ & $P(7)$ & $\mathrm{C}(97)$ & $104.7(3)$ & $P(7)$ & $\mathrm{C}(97)$ & $\mathrm{C}(98)$ & $121.7(5)$ \\
\hline$P(7)$ & $C(97)$ & $C(102)$ & $121.0(5)$ & $P(7)$ & $C(103)$ & $C(104)$ & 123.3(5) \\
\hline$P(7)$ & $C(103)$ & $C(108)$ & 119.1(4) & $P(8)$ & $C(96)$ & $C(91)$ & $118.0(4)$ \\
\hline C(109) & $\mathrm{P}(8)$ & $C(96)$ & 103.2(3) & $\mathrm{P}(8)$ & $C(96)$ & C(95) & 123.1(5) \\
\hline$C(115)$ & $P(8)$ & $C(96)$ & 104.6(3) & $P(8)$ & $C(109)$ & $C(110)$ & $125.4(5)$ \\
\hline$P(8)$ & $C(109)$ & $C(114)$ & $118.1(5)$ & $C(115)$ & $P(8)$ & $C(109)$ & $104.2(3)$ \\
\hline$P(8)$ & $C(115)$ & $C(116)$ & $124.4(5)$ & $P(8)$ & $C(115)$ & $C(120)$ & 118.3(5) \\
\hline$C(6)$ & $C(1)$ & $C(2)$ & $119.8(5)$ & $C(1)$ & $C(2)$ & $C(3)$ & $120.7(6)$ \\
\hline$C(1)$ & $C(6)$ & $C(5)$ & 118.2(6) & $C(2)$ & $C(3)$ & $C(4)$ & $119.3(7)$ \\
\hline$C(3)$ & $C(4)$ & $C(5)$ & $120.5(6)$ & $C(4)$ & $C(5)$ & $C(6)$ & $121.5(7)$ \\
\hline$C(12)$ & $C(7)$ & $C(8)$ & $118.0(6)$ & $C(7)$ & $C(8)$ & $\mathrm{C}(9)$ & $120.0(9)$ \\
\hline C(7) & $C(12)$ & $C(11)$ & $122.7(6)$ & $C(8)$ & $\mathrm{C}(9)$ & $C(10)$ & 119.6(9) \\
\hline $\mathrm{C}(9)$ & $C(10)$ & $C(11)$ & $120.1(8)$ & $C(10)$ & $C(11)$ & $C(12)$ & $119.3(7)$ \\
\hline$C(18)$ & $C(13)$ & $C(14)$ & $117.3(6)$ & $C(13)$ & $C(14)$ & $C(15)$ & $120.8(6)$ \\
\hline$C(13)$ & $C(18)$ & $C(17)$ & $121.8(6)$ & $C(14)$ & $C(15)$ & $C(16)$ & $120.2(7)$ \\
\hline$C(15)$ & $C(16)$ & $C(17)$ & $120.3(7)$ & $C(16)$ & $C(17)$ & $C(18)$ & $119.5(7)$ \\
\hline$C(24)$ & $C(19)$ & $C(20)$ & $116.5(7)$ & $C(19)$ & $C(20)$ & $C(21)$ & $120.7(8)$ \\
\hline$C(19)$ & $C(24)$ & $C(23)$ & $121.8(8)$ & $C(20)$ & $C(21)$ & $C(22)$ & 122(1) \\
\hline$C(21)$ & $C(22)$ & $C(23)$ & 117.1(9) & $C(22)$ & $\mathrm{C}(23)$ & $C(24)$ & $121.4(8)$ \\
\hline
\end{tabular}

Table 6. Bond angles $\left({ }^{\circ}\right)$-- continued 


\begin{tabular}{|c|c|c|c|c|c|c|c|}
\hline atom & atom & atom & angle & atom & atom & atom & angle \\
\hline$C(30)$ & $C(25)$ & $C(26)$ & $117.9(6)$ & $C(25)$ & $C(26)$ & $C(27)$ & $121.5(7)$ \\
\hline$C(25)$ & $C(30)$ & $C(29)$ & $119.8(7)$ & $C(26)$ & $C(27)$ & $C(28)$ & $119.0(7)$ \\
\hline$C(27)$ & $\mathrm{C}(28)$ & C(29) & 119.7(8) & $C(28)$ & $C(29)$ & $C(30)$ & $122.0(8)$ \\
\hline$C(36)$ & $C(31)$ & $C(32)$ & 118.6(6) & $C(31)$ & $C(32)$ & $C(33)$ & $121.7(7)$ \\
\hline$C(31)$ & $C(36)$ & $C(35)$ & $120.2(5)$ & $C(32)$ & $C(33)$ & $C(34)$ & $119.5(6)$ \\
\hline$C(33)$ & $C(34)$ & $C(35)$ & 119.7(6) & $C(34)$ & $C(35)$ & $C(36)$ & $120.2(7)$ \\
\hline$C(42)$ & $C(37)$ & $C(38)$ & 116.4(8) & $C(37)$ & $C(38)$ & $C(39)$ & $120(1)$ \\
\hline$C(37)$ & C(42) & $C(41)$ & $122.4(9)$ & $C(38)$ & $C(39)$ & $C(40)$ & $121(1)$ \\
\hline$C(39)$ & $C(40)$ & $C(41)$ & 119(1) & $C(40)$ & $C(41)$ & $C(42)$ & $119(1)$ \\
\hline$C(48)$ & $C(43)$ & C(44) & $117.7(6)$ & $C(43)$ & C(44) & $C(45)$ & $122.4(7)$ \\
\hline$C(43)$ & $C(48)$ & $\mathrm{C}(47)$ & $119.7(7)$ & $C(44)$ & $C(45)$ & $C(46)$ & 119.7(8) \\
\hline$C(45)$ & $C(46)$ & $C(47)$ & $118.4(8)$ & $C(46)$ & $C(47)$ & $C(48)$ & $122.0(8)$ \\
\hline$C(54)$ & C(49) & $C(50)$ & $116.0(7)$ & $C(49)$ & $C(50)$ & $C(51)$ & 122.7(8) \\
\hline$C(49)$ & $C(54)$ & $C(53)$ & $121.2(8)$ & $C(50)$ & $C(51)$ & $C(52)$ & 119.9(9) \\
\hline$C(51)$ & $C(52)$ & $C(53)$ & $119(1)$ & $C(52)$ & $C(53)$ & $C(54)$ & $120(1)$ \\
\hline$C(60)$ & $C(55)$ & $C(56)$ & $117.4(6)$ & $C(55)$ & $C(56)$ & $C(57)$ & $121.5(7)$ \\
\hline$C(55)$ & $C(60)$ & $C(59)$ & $119.9(6)$ & $C(56)$ & $C(57)$ & $C(58)$ & $120.8(7)$ \\
\hline$C(57)$ & $C(58)$ & $C(59)$ & 119.4(8) & $C(58)$ & $C(59)$ & $C(60)$ & $120.9(9)$ \\
\hline$C(66)$ & $C(61)$ & $C(62)$ & $118.7(5)$ & $C(61)$ & $C(62)$ & $C(63)$ & $120.5(6)$ \\
\hline$C(61)$ & $C(66)$ & $C(65)$ & $119.3(5)$ & $C(62)$ & C(63) & $C(64)$ & $120.8(6)$ \\
\hline$C(63)$ & C(64) & $C(65)$ & 119.4(6) & $C(64)$ & $C(65)$ & $C(66)$ & $121.3(6)$ \\
\hline$C(72)$ & C(67) & $C(68)$ & $117.7(5)$ & C(67) & C(68) & $C(69)$ & $120.8(6)$ \\
\hline$C(67)$ & C(72) & $C(71)$ & $121.7(6)$ & C(68) & $C(69)$ & $C(70)$ & $119.9(6)$ \\
\hline $\mathrm{C}(69)$ & $C(70)$ & $C(71)$ & $120.4(7)$ & $C(70)$ & $C(71)$ & $C(72)$ & 119.4(7) \\
\hline$C(78)$ & $C(73)$ & $C(74)$ & $115.4(7)$ & $C(73)$ & $C(74)$ & $C(75)$ & $122.2(8)$ \\
\hline$C(73)$ & $C(78)$ & $C(77)$ & $122.8(7)$ & $C(74)$ & $C(75)$ & $C(76)$ & 120.3(9) \\
\hline$C(75)$ & $C(76)$ & $C(77)$ & 118.4(9) & $C(76)$ & $C(77)$ & $C(78)$ & $120.8(9)$ \\
\hline$C(84)$ & C(79) & $C(80)$ & $115.1(6)$ & C(79) & $\mathrm{C}(80)$ & $C(81)$ & $122.9(7)$ \\
\hline$C(79)$ & $C(84)$ & $C(83)$ & $121.6(8)$ & $C(80)$ & $C(81)$ & $C(82)$ & $119.7(8)$ \\
\hline$C(81)$ & $C(82)$ & $C(83)$ & $118.9(9)$ & $C(82)$ & C(83) & $C(84)$ & 121.7(9) \\
\hline$C(90)$ & C(85) & $C(86)$ & $118.5(6)$ & $C(85)$ & $C(86)$ & $C(87)$ & $121.2(7)$ \\
\hline$C(85)$ & $C(90)$ & $C(89)$ & $120.4(7)$ & $C(86)$ & $C(87)$ & $C(88)$ & $118.8(7)$ \\
\hline$C(87)$ & $C(88)$ & $C(89)$ & $121.2(8)$ & $C(88)$ & $C(89)$ & $C(90)$ & $120.0(8)$ \\
\hline$C(96)$ & $C(91)$ & $C(92)$ & 118.6(6) & $C(91)$ & $C(92)$ & $C(93)$ & $120.9(7)$ \\
\hline$C(91)$ & $C(96)$ & $C(95)$ & $118.9(5)$ & $C(92)$ & $C(93)$ & $C(94)$ & $120.2(6)$ \\
\hline$C(93)$ & $C(94)$ & $C(95)$ & $119.7(7)$ & $C(94)$ & $C(95)$ & $C(96)$ & $121.6(7)$ \\
\hline$C(102)$ & $\mathrm{C}(97)$ & C(98) & $116.6(6)$ & $C(97)$ & $C(98)$ & C(99) & $122.0(7)$ \\
\hline
\end{tabular}

Table 6. Bond angles $\left({ }^{\circ}\right)$-- continued

$\begin{array}{llllllll}\text { atom } & \text { atom } & \text { atom } & \text { angle } & \text { atom } & \text { atom } & \text { atom } & \text { angle } \\ \mathrm{C}(97) & \mathrm{C}(102) & \mathrm{C}(101) & 120.9(7) & \mathrm{C}(98) & \mathrm{C}(99) & \mathrm{C}(100) & 120.5(8) \\ \mathrm{C}(99) & \mathrm{C}(100) & \mathrm{C}(101) & 119.1(8) & \mathrm{C}(100) & \mathrm{C}(101) & \mathrm{C}(102) & 120.9(8) \\ \mathrm{C}(108) & \mathrm{C}(103) & \mathrm{C}(104) & 117.3(6) & \mathrm{C}(103) & \mathrm{C}(104) & \mathrm{C}(105) & 120.4(7) \\ \mathrm{C}(103) & \mathrm{C}(108) & \mathrm{C}(107) & 122.3(6) & \mathrm{C}(104) & \mathrm{C}(105) & \mathrm{C}(106) & 120.8(7) \\ \mathrm{C}(105) & \mathrm{C}(106) & \mathrm{C}(107) & 120.6(7) & \mathrm{C}(106) & \mathrm{C}(107) & \mathrm{C}(108) & 118.4(7) \\ \mathrm{C}(114) & \mathrm{C}(109) & \mathrm{C}(110) & 116.4(6) & \mathrm{C}(109) & \mathrm{C}(110) & \mathrm{C}(111) & 121.8(7) \\ \mathrm{C}(109) & \mathrm{C}(114) & \mathrm{C}(113) & 121.6(7) & \mathrm{C}(110) & \mathrm{C}(111) & \mathrm{C}(112) & 119.5(8) \\ \mathrm{C}(111) & \mathrm{C}(112) & \mathrm{C}(113) & 120.9(8) & \mathrm{C}(112) & \mathrm{C}(113) & \mathrm{C}(114) & 119.6(9) \\ \mathrm{C}(120) & \mathrm{C}(115) & \mathrm{C}(116) & 117.3(6) & \mathrm{C}(115) & \mathrm{C}(116) & \mathrm{C}(117) & 122.0(7)\end{array}$


$\mathrm{C}(115) \quad \mathrm{C}(120) \quad \mathrm{C}(119) \quad 120.3(7)$

$\mathrm{C}(116) \quad \mathrm{C}(117) \quad \mathrm{C}(118) \quad 119.7(8)$

$C(117) \quad C(118) \quad C(119) \quad 119.3(9)$

$\mathrm{C}(118) \quad \mathrm{C}(119) \quad \mathrm{C}(120) \quad 121.3(8)$ 
Table 8. Torsion Angles $\left({ }^{\circ}\right)$

\begin{tabular}{|c|c|c|c|c|c|c|c|c|c|}
\hline atom1 & atom2 & atom3 & atom4 & angle & atom1 & atom2 & atom3 & atom4 & angle \\
\hline $\mathrm{Cu}(2)$ & $\mathrm{I}(1)$ & $\mathrm{Cu}(1)$ & $\mathrm{I}(2)$ & $21.43(3)$ & $\mathrm{Cu}(2)$ & $\mathrm{I}(1)$ & $\mathrm{Cu}(1)$ & $P(1)$ & $146.64(7)$ \\
\hline $\mathrm{Cu}(2)$ & $\mathrm{I}(1)$ & $\mathrm{Cu}(1)$ & $P(2)$ & $-105.18(6)$ & $\mathrm{Cu}(1)$ & $\mathrm{I}(1)$ & $\mathrm{Cu}(2)$ & $\mathrm{I}(2)$ & $-22.27(4)$ \\
\hline $\mathrm{Cu}(1)$ & $I(1)$ & $\mathrm{Cu}(2)$ & $P(3)$ & $-156.00(6)$ & $\mathrm{Cu}(1)$ & $\mathrm{I}(1)$ & $\mathrm{Cu}(2)$ & $P(4)$ & $110.71(6)$ \\
\hline $\mathrm{Cu}(2)$ & $\mathrm{I}(2)$ & $\mathrm{Cu}(1)$ & $\mathrm{I}(1)$ & $-21.93(3)$ & $\mathrm{Cu}(2)$ & $\mathrm{I}(2)$ & $\mathrm{Cu}(1)$ & $P(1)$ & $-151.78(6)$ \\
\hline $\mathrm{Cu}(2)$ & $\mathrm{I}(2)$ & $\mathrm{Cu}(1)$ & $P(2)$ & 113.66(5) & $\mathrm{Cu}(1)$ & $\mathrm{I}(2)$ & $\mathrm{Cu}(2)$ & $\mathrm{I}(1)$ & $21.77(4)$ \\
\hline $\mathrm{Cu}(1)$ & $\mathrm{I}(2)$ & $\mathrm{Cu}(2)$ & $P(3)$ & $148.24(7)$ & $\mathrm{Cu}(1)$ & $\mathrm{I}(2)$ & $\mathrm{Cu}(2)$ & $P(4)$ & $-107.93(6)$ \\
\hline $\mathrm{Cu}(4)$ & I(3) & $\mathrm{Cu}(3)$ & $\mathrm{I}(4)$ & $20.50(3)$ & $\mathrm{Cu}(4)$ & $\mathrm{I}(3)$ & $\mathrm{Cu}(3)$ & $P(5)$ & $143.51(7)$ \\
\hline $\mathrm{Cu}(4)$ & I(3) & $\mathrm{Cu}(3)$ & $P(6)$ & $-107.70(5)$ & $\mathrm{Cu}(3)$ & $\mathrm{I}(3)$ & $\mathrm{Cu}(4)$ & $\mathrm{I}(4)$ & $-21.64(4)$ \\
\hline $\mathrm{Cu}(3)$ & $\mathrm{I}(3)$ & $\mathrm{Cu}(4)$ & $P(7)$ & $-151.08(6)$ & $\mathrm{Cu}(3)$ & $\mathrm{I}(3)$ & $\mathrm{Cu}(4)$ & $P(8)$ & $117.22(6)$ \\
\hline $\mathrm{Cu}(4)$ & $\mathrm{I}(4)$ & $\mathrm{Cu}(3)$ & I(3) & $-21.12(3)$ & $\mathrm{Cu}(4)$ & $\mathrm{I}(4)$ & $\mathrm{Cu}(3)$ & $P(5)$ & $-152.98(6)$ \\
\hline $\mathrm{Cu}(4)$ & $\mathrm{I}(4)$ & $\mathrm{Cu}(3)$ & $P(6)$ & $112.46(5)$ & $\mathrm{Cu}(3)$ & $\mathrm{I}(4)$ & $\mathrm{Cu}(4)$ & $\mathrm{I}(3)$ & $21.08(4)$ \\
\hline $\mathrm{Cu}(3)$ & $\mathrm{I}(4)$ & $\mathrm{Cu}(4)$ & $P(7)$ & $142.26(6)$ & $\mathrm{Cu}(3)$ & $\mathrm{I}(4)$ & $\mathrm{Cu}(4)$ & $P(8)$ & $-112.31(7)$ \\
\hline $\mathrm{I}(1)$ & $\mathrm{Cu}(1)$ & $P(1)$ & $C(6)$ & 153.1(2) & $\mathrm{I}(1)$ & $\mathrm{Cu}(1)$ & $P(1)$ & $C(7)$ & 39.8(2) \\
\hline $\mathrm{I}(1)$ & $\mathrm{Cu}(1)$ & $P(1)$ & $C(13)$ & $-89.1(3)$ & $\mathrm{I}(2)$ & $\mathrm{Cu}(1)$ & $P(1)$ & $C(6)$ & $-82.1(2)$ \\
\hline I(2) & $\mathrm{Cu}(1)$ & $P(1)$ & $\mathrm{C}(7)$ & $164.6(2)$ & $\mathrm{I}(2)$ & $\mathrm{Cu}(1)$ & $P(1)$ & $C(13)$ & $35.7(3)$ \\
\hline$P(2)$ & $\mathrm{Cu}(1)$ & $P(1)$ & $C(6)$ & $25.5(2)$ & $P(2)$ & $\mathrm{Cu}(1)$ & $P(1)$ & $C(7)$ & $-87.8(2)$ \\
\hline$P(2)$ & $\mathrm{Cu}(1)$ & $P(1)$ & $C(13)$ & 143.3(3) & $\mathrm{I}(1)$ & $\mathrm{Cu}(1)$ & $P(2)$ & $C(1)$ & $-148.1(2)$ \\
\hline $\mathrm{I}(1)$ & $\mathrm{Cu}(1)$ & $P(2)$ & $C(19)$ & $-36.2(2)$ & $\mathrm{I}(1)$ & $\mathrm{Cu}(1)$ & $P(2)$ & $C(25)$ & $97.5(2)$ \\
\hline $\mathrm{I}(2)$ & $\mathrm{Cu}(1)$ & $P(2)$ & C(1) & $84.6(2)$ & $\mathrm{I}(2)$ & $\mathrm{Cu}(1)$ & $P(2)$ & $C(19)$ & $-163.5(2)$ \\
\hline $\mathrm{I}(2)$ & $\mathrm{Cu}(1)$ & $P(2)$ & $C(25)$ & $-29.9(2)$ & $P(1)$ & $\mathrm{Cu}(1)$ & $\mathrm{P}(2)$ & $C(1)$ & $-25.4(2)$ \\
\hline$P(1)$ & $\mathrm{Cu}(1)$ & $\mathrm{P}(2)$ & $C(19)$ & $86.5(2)$ & $P(1)$ & $\mathrm{Cu}(1)$ & $\mathrm{P}(2)$ & $C(25)$ & $-139.9(2)$ \\
\hline I(1) & $\mathrm{Cu}(2)$ & $P(3)$ & C(31) & $-78.0(2)$ & $\mathrm{I}(1)$ & $\mathrm{Cu}(2)$ & $P(3)$ & $\mathrm{C}(37)$ & $37.8(3)$ \\
\hline $\mathrm{I}(1)$ & $\mathrm{Cu}(2)$ & $P(3)$ & $C(43)$ & 172.3(2) & $\mathrm{I}(2)$ & $\mathrm{Cu}(2)$ & $P(3)$ & $\mathrm{C}(31)$ & 154.5(2) \\
\hline $\mathrm{I}(2)$ & $\mathrm{Cu}(2)$ & $P(3)$ & C(37) & $-89.7(3)$ & $\mathrm{I}(2)$ & $\mathrm{Cu}(2)$ & $P(3)$ & $C(43)$ & $44.8(3)$ \\
\hline$P(4)$ & $\mathrm{Cu}(2)$ & $P(3)$ & C(31) & $33.7(2)$ & $P(4)$ & $\mathrm{Cu}(2)$ & $P(3)$ & $\mathrm{C}(37)$ & 149.5(3) \\
\hline$P(4)$ & $\mathrm{Cu}(2)$ & $P(3)$ & $C(43)$ & $-75.9(3)$ & $\mathrm{I}(1)$ & $\mathrm{Cu}(2)$ & $P(4)$ & $C(36)$ & $75.8(2)$ \\
\hline I(1) & $\mathrm{Cu}(2)$ & $P(4)$ & $C(49)$ & $-41.1(3)$ & $\mathrm{I}(1)$ & $\mathrm{Cu}(2)$ & $\mathrm{P}(4)$ & $C(55)$ & $-173.0(2)$ \\
\hline $\mathrm{I}(2)$ & $\mathrm{Cu}(2)$ & $P(4)$ & $C(36)$ & $-155.4(2)$ & I(2) & $\mathrm{Cu}(2)$ & $P(4)$ & $\mathrm{C}(49)$ & $87.8(3)$ \\
\hline I(2) & $\mathrm{Cu}(2)$ & $P(4)$ & $C(55)$ & $-44.1(2)$ & $P(3)$ & $\mathrm{Cu}(2)$ & $\mathrm{P}(4)$ & $C(36)$ & $-31.8(2)$ \\
\hline$P(3)$ & $\mathrm{Cu}(2)$ & $P(4)$ & $C(49)$ & $-148.6(3)$ & $P(3)$ & $\mathrm{Cu}(2)$ & $P(4)$ & $C(55)$ & $79.4(2)$ \\
\hline $\mathrm{I}(3)$ & $\mathrm{Cu}(3)$ & $P(5)$ & $C(61)$ & $148.7(2)$ & $\mathrm{I}(3)$ & $\mathrm{Cu}(3)$ & $P(5)$ & $C(67)$ & $35.6(2)$ \\
\hline I(3) & $\mathrm{Cu}(3)$ & $P(5)$ & C(73) & $-94.7(3)$ & $\mathrm{I}(4)$ & $\mathrm{Cu}(3)$ & $P(5)$ & $C(61)$ & $-86.4(2)$ \\
\hline I(4) & $\mathrm{Cu}(3)$ & $P(5)$ & C(67) & $160.5(2)$ & $\mathrm{I}(4)$ & $\mathrm{Cu}(3)$ & $P(5)$ & $C(73)$ & $30.2(3)$ \\
\hline$P(6)$ & $\mathrm{Cu}(3)$ & $P(5)$ & $C(61)$ & $23.9(2)$ & $P(6)$ & $\mathrm{Cu}(3)$ & $P(5)$ & $\mathrm{C}(67)$ & $-89.2(2)$ \\
\hline$P(6)$ & $\mathrm{Cu}(3)$ & $P(5)$ & $C(73)$ & $140.5(3)$ & I(3) & $\mathrm{Cu}(3)$ & $P(6)$ & $C(66)$ & $-148.7(2)$ \\
\hline I(3) & $\mathrm{Cu}(3)$ & $P(6)$ & $C(79)$ & $-35.2(2)$ & $\mathrm{I}(3)$ & $\mathrm{Cu}(3)$ & $P(6)$ & C(85) & $96.4(2)$ \\
\hline $\mathrm{I}(4)$ & $\mathrm{Cu}(3)$ & $P(6)$ & $C(66)$ & $83.2(2)$ & $\mathrm{I}(4)$ & $\mathrm{Cu}(3)$ & $P(6)$ & C(79) & $-163.2(2)$ \\
\hline
\end{tabular}

Table 8. Torsion angles $\left({ }^{\circ}\right)$-- continued

$\begin{array}{llllllllll}\text { atom1 } & \text { atom2 } & \text { atom3 } & \text { atom4 } & \text { angle } & \text { atom1 } & \text { atom2 } & \text { atom3 } & \text { atom4 } & \text { angle } \\ \mathrm{I}(4) & \mathrm{Cu}(3) & \mathrm{P}(6) & \mathrm{C}(85) & -31.7(2) & \mathrm{P}(5) & \mathrm{Cu}(3) & \mathrm{P}(6) & \mathrm{C}(66) & -22.9(2) \\ \mathrm{P}(5) & \mathrm{Cu}(3) & \mathrm{P}(6) & \mathrm{C}(79) & 90.7(2) & \mathrm{P}(5) & \mathrm{Cu}(3) & \mathrm{P}(6) & \mathrm{C}(85) & -137.8(2) \\ \mathrm{I}(3) & \mathrm{Cu}(4) & \mathrm{P}(7) & \mathrm{C}(91) & -77.3(2) & \mathrm{I}(3) & \mathrm{Cu}(4) & \mathrm{P}(7) & \mathrm{C}(97) & 40.7(3) \\ \mathrm{I}(3) & \mathrm{Cu}(4) & \mathrm{P}(7) & \mathrm{C}(103) & 173.6(2) & \mathrm{I}(4) & \mathrm{Cu}(4) & \mathrm{P}(7) & \mathrm{C}(91) & 157.9(2) \\ \mathrm{I}(4) & \mathrm{Cu}(4) & \mathrm{P}(7) & \mathrm{C}(97) & -84.2(3) & \mathrm{I}(4) & \mathrm{Cu}(4) & \mathrm{P}(7) & \mathrm{C}(103) & 48.8(2)\end{array}$




\begin{tabular}{|c|c|c|c|c|c|c|c|c|c|}
\hline $\mathrm{P}(8)$ & $\mathrm{Cu}(4)$ & $P(7)$ & $C(91)$ & $32.9(2)$ & $P(8)$ & $\mathrm{Cu}(4)$ & $\mathrm{P}(7)$ & $\mathrm{C}(97)$ & $150.9(3)$ \\
\hline$P(8)$ & $\mathrm{Cu}(4)$ & $P(7)$ & $C(103)$ & $-76.2(2)$ & $\mathrm{I}(3)$ & $\mathrm{Cu}(4)$ & $P(8)$ & $C(96)$ & $71.7(2)$ \\
\hline I(3) & $\mathrm{Cu}(4)$ & $P(8)$ & $C(109)$ & $-177.6(2)$ & I(3) & $\mathrm{Cu}(4)$ & $P(8)$ & $C(115)$ & $-45.5(3)$ \\
\hline I(4) & $\mathrm{Cu}(4)$ & $P(8)$ & $C(96)$ & $-154.4(2)$ & I(4) & $\mathrm{Cu}(4)$ & $P(8)$ & $C(109)$ & $-43.7(2)$ \\
\hline $\mathrm{I}(4)$ & $\mathrm{Cu}(4)$ & $P(8)$ & $C(115)$ & $88.4(3)$ & $\mathrm{P}(7)$ & $\mathrm{Cu}(4)$ & $P(8)$ & $C(96)$ & $-32.1(2)$ \\
\hline$P(7)$ & $\mathrm{Cu}(4)$ & $P(8)$ & $C(109)$ & 78.6(2) & $P(7)$ & $\mathrm{Cu}(4)$ & $P(8)$ & $C(115)$ & $-149.3(3)$ \\
\hline $\mathrm{Cu}(1)$ & $P(1)$ & $C(6)$ & $C(1)$ & $-20.5(5)$ & $\mathrm{Cu}(1)$ & $P(1)$ & $C(6)$ & C(5) & $160.6(5)$ \\
\hline$C(7)$ & $P(1)$ & $C(6)$ & $C(1)$ & $99.5(5)$ & $C(7)$ & $P(1)$ & $C(6)$ & $C(5)$ & $-79.3(5)$ \\
\hline C(13) & $P(1)$ & $C(6)$ & $C(1)$ & $-152.1(4)$ & $C(13)$ & $P(1)$ & $C(6)$ & $C(5)$ & $29.0(5)$ \\
\hline $\mathrm{Cu}(1)$ & $P(1)$ & $C(7)$ & $C(8)$ & $121.6(6)$ & $\mathrm{Cu}(1)$ & $P(1)$ & $C(7)$ & $C(12)$ & $-57.6(6)$ \\
\hline$C(6)$ & $P(1)$ & $C(7)$ & $C(8)$ & $9.9(7)$ & $C(6)$ & $P(1)$ & $C(7)$ & $C(12)$ & $-169.3(5)$ \\
\hline$C(13)$ & $P(1)$ & $C(7)$ & $C(8)$ & $-99.3(7)$ & $C(13)$ & $P(1)$ & $C(7)$ & $C(12)$ & $81.5(5)$ \\
\hline $\mathrm{Cu}(1)$ & $P(1)$ & $C(13)$ & $C(14)$ & $164.8(5)$ & $\mathrm{Cu}(1)$ & $P(1)$ & $C(13)$ & $C(18)$ & $-12.5(6)$ \\
\hline$C(6)$ & $P(1)$ & $C(13)$ & $C(14)$ & $-78.4(6)$ & $C(6)$ & $P(1)$ & $C(13)$ & $C(18)$ & $104.4(5)$ \\
\hline$C(7)$ & $P(1)$ & $C(13)$ & $C(14)$ & $31.4(6)$ & $C(7)$ & $P(1)$ & $C(13)$ & $C(18)$ & $-145.8(5)$ \\
\hline $\mathrm{Cu}(1)$ & $P(2)$ & $C(1)$ & $C(2)$ & $-158.3(5)$ & $\mathrm{Cu}(1)$ & $P(2)$ & $C(1)$ & $C(6)$ & $20.7(5)$ \\
\hline$C(19)$ & $P(2)$ & $C(1)$ & $C(2)$ & $78.1(5)$ & $C(19)$ & $P(2)$ & $C(1)$ & $C(6)$ & $-102.9(5)$ \\
\hline$C(25)$ & $P(2)$ & $C(1)$ & $C(2)$ & $-31.2(5)$ & $C(25)$ & $P(2)$ & $C(1)$ & $C(6)$ & $147.7(4)$ \\
\hline $\mathrm{Cu}(1)$ & $P(2)$ & $C(19)$ & $C(20)$ & $23.8(6)$ & $\mathrm{Cu}(1)$ & $P(2)$ & $C(19)$ & $C(24)$ & $-159.1(5)$ \\
\hline$C(1)$ & $P(2)$ & $C(19)$ & $C(20)$ & $135.5(5)$ & $C(1)$ & $P(2)$ & $C(19)$ & $C(24)$ & $-47.4(6)$ \\
\hline C(25) & $P(2)$ & $C(19)$ & $C(20)$ & $-116.5(5)$ & $C(25)$ & $P(2)$ & $C(19)$ & $C(24)$ & $60.7(6)$ \\
\hline $\mathrm{Cu}(1)$ & $P(2)$ & $C(25)$ & $C(26)$ & $56.4(5)$ & $\mathrm{Cu}(1)$ & $P(2)$ & $C(25)$ & $C(30)$ & $-119.4(5)$ \\
\hline$C(1)$ & $P(2)$ & $C(25)$ & $C(26)$ & $-57.4(5)$ & $C(1)$ & $P(2)$ & $C(25)$ & $C(30)$ & $126.9(5)$ \\
\hline C(19) & $P(2)$ & $C(25)$ & $C(26)$ & $-164.7(5)$ & $C(19)$ & $P(2)$ & $C(25)$ & $C(30)$ & $19.5(6)$ \\
\hline $\mathrm{Cu}(2)$ & $P(3)$ & $C(31)$ & $C(32)$ & $152.9(4)$ & $\mathrm{Cu}(2)$ & $P(3)$ & $C(31)$ & $C(36)$ & $-29.9(5)$ \\
\hline C(37) & $P(3)$ & $C(31)$ & $C(32)$ & $23.8(5)$ & $C(37)$ & $P(3)$ & $C(31)$ & $C(36)$ & $-159.1(4)$ \\
\hline$C(43)$ & $P(3)$ & $C(31)$ & $C(32)$ & $-86.3(5)$ & $C(43)$ & $P(3)$ & $C(31)$ & $C(36)$ & $90.8(4)$ \\
\hline $\mathrm{Cu}(2)$ & $P(3)$ & $C(37)$ & $C(38)$ & $118.7(6)$ & $\mathrm{Cu}(2)$ & $P(3)$ & $C(37)$ & $C(42)$ & $-57.0(6)$ \\
\hline$C(31)$ & $P(3)$ & $C(37)$ & $C(38)$ & $-127.6(6)$ & $C(31)$ & $P(3)$ & $C(37)$ & $C(42)$ & $56.7(6)$ \\
\hline$C(43)$ & $P(3)$ & $C(37)$ & $C(38)$ & $-20.0(6)$ & $C(43)$ & $P(3)$ & $C(37)$ & $C(42)$ & $164.4(5)$ \\
\hline $\mathrm{Cu}(2)$ & $P(3)$ & $C(43)$ & $C(44)$ & $-21.9(7)$ & $\mathrm{Cu}(2)$ & $P(3)$ & $C(43)$ & $C(48)$ & $156.6(6)$ \\
\hline$C(31)$ & $P(3)$ & $C(43)$ & $C(44)$ & $-130.6(6)$ & $C(31)$ & $P(3)$ & $C(43)$ & $C(48)$ & $48.0(7)$ \\
\hline
\end{tabular}

Table 8. Torsion angles $\left(^{\circ}\right)$-- continued

$\begin{array}{llllllllll}\text { atom1 } & \text { atom2 } & \text { atom3 } & \text { atom4 } & \text { angle } & \text { atom1 } & \text { atom2 } & \text { atom3 } & \text { atom4 } & \text { angle } \\ \mathrm{C}(37) & \mathrm{P}(3) & \mathrm{C}(43) & \mathrm{C}(44) & 120.0(6) & \mathrm{C}(37) & \mathrm{P}(3) & \mathrm{C}(43) & \mathrm{C}(48) & -61.5(6) \\ \mathrm{Cu}(2) & \mathrm{P}(4) & \mathrm{C}(36) & \mathrm{C}(31) & 23.3(5) & \mathrm{Cu}(2) & \mathrm{P}(4) & \mathrm{C}(36) & \mathrm{C}(35) & -156.6(4) \\ \mathrm{C}(49) & \mathrm{P}(4) & \mathrm{C}(36) & \mathrm{C}(31) & 154.6(4) & \mathrm{C}(49) & \mathrm{P}(4) & \mathrm{C}(36) & \mathrm{C}(35) & -25.2(5) \\ \mathrm{C}(55) & \mathrm{P}(4) & \mathrm{C}(36) & \mathrm{C}(31) & -95.0(5) & \mathrm{C}(55) & \mathrm{P}(4) & \mathrm{C}(36) & \mathrm{C}(35) & 85.2(5) \\ \mathrm{Cu}(2) & \mathrm{P}(4) & \mathrm{C}(49) & \mathrm{C}(50) & -142.8(5) & \mathrm{Cu}(2) & \mathrm{P}(4) & \mathrm{C}(49) & \mathrm{C}(54) & 34.7(6) \\ \mathrm{C}(36) & \mathrm{P}(4) & \mathrm{C}(49) & \mathrm{C}(50) & 101.9(6) & \mathrm{C}(36) & \mathrm{P}(4) & \mathrm{C}(49) & \mathrm{C}(54) & -80.5(5) \\ \mathrm{C}(55) & \mathrm{P}(4) & \mathrm{C}(49) & \mathrm{C}(50) & -7.8(6) & \mathrm{C}(55) & \mathrm{P}(4) & \mathrm{C}(49) & \mathrm{C}(54) & 169.8(5) \\ \mathrm{Cu}(2) & \mathrm{P}(4) & \mathrm{C}(55) & \mathrm{C}(56) & -117.0(6) & \mathrm{Cu}(2) & \mathrm{P}(4) & \mathrm{C}(55) & \mathrm{C}(60) & 56.5(6) \\ \mathrm{C}(36) & \mathrm{P}(4) & \mathrm{C}(55) & \mathrm{C}(56) & -7.9(7) & \mathrm{C}(36) & \mathrm{P}(4) & \mathrm{C}(55) & \mathrm{C}(60) & 165.7(5) \\ \mathrm{C}(49) & \mathrm{P}(4) & \mathrm{C}(55) & \mathrm{C}(56) & 101.9(6) & \mathrm{C}(49) & \mathrm{P}(4) & \mathrm{C}(55) & \mathrm{C}(60) & -84.5(6) \\ \mathrm{Cu}(3) & \mathrm{P}(5) & \mathrm{C}(61) & \mathrm{C}(62) & 160.2(4) & \mathrm{Cu}(3) & \mathrm{P}(5) & \mathrm{C}(61) & \mathrm{C}(66) & -20.6(5) \\ \mathrm{C}(67) & \mathrm{P}(5) & \mathrm{C}(61) & \mathrm{C}(62) & -78.4(5) & \mathrm{C}(67) & \mathrm{P}(5) & \mathrm{C}(61) & \mathrm{C}(66) & 100.8(4) \\ \mathrm{C}(73) & \mathrm{P}(5) & \mathrm{C}(61) & \mathrm{C}(62) & 29.6(5) & \mathrm{C}(73) & \mathrm{P}(5) & \mathrm{C}(61) & \mathrm{C}(66) & -151.2(4) \\ \mathrm{Cu}(3) & \mathrm{P}(5) & \mathrm{C}(67) & \mathrm{C}(68) & 137.9(5) & \mathrm{Cu}(3) & \mathrm{P}(5) & \mathrm{C}(67) & \mathrm{C}(72) & -41.6(6) \\ \mathrm{C}(61) & \mathrm{P}(5) & \mathrm{C}(67) & \mathrm{C}(68) & 26.0(6) & \mathrm{C}(61) & \mathrm{P}(5) & \mathrm{C}(67) & \mathrm{C}(72) & -153.5(5)\end{array}$




$\begin{array}{lllll}\mathrm{C}(73) & \mathrm{P}(5) & \mathrm{C}(67) & \mathrm{C}(68) & -82.3(6) \\ \mathrm{Cu}(3) & \mathrm{P}(5) & \mathrm{C}(73) & \mathrm{C}(74) & 131.9(6) \\ \mathrm{C}(61) & \mathrm{P}(5) & \mathrm{C}(73) & \mathrm{C}(74) & -112.2(6) \\ \mathrm{C}(67) & \mathrm{P}(5) & \mathrm{C}(73) & \mathrm{C}(74) & -3.3(7) \\ \mathrm{Cu}(3) & \mathrm{P}(6) & \mathrm{C}(66) & \mathrm{C}(61) & 17.3(5) \\ \mathrm{C}(79) & \mathrm{P}(6) & \mathrm{C}(66) & \mathrm{C}(61) & -108.2(4) \\ \mathrm{C}(85) & \mathrm{P}(6) & \mathrm{C}(66) & \mathrm{C}(61) & 142.8(4) \\ \mathrm{Cu}(3) & \mathrm{P}(6) & \mathrm{C}(79) & \mathrm{C}(80) & -169.3(5) \\ \mathrm{C}(66) & \mathrm{P}(6) & \mathrm{C}(79) & \mathrm{C}(80) & -56.1(6) \\ \mathrm{C}(85) & \mathrm{P}(6) & \mathrm{C}(79) & \mathrm{C}(80) & 52.9(6) \\ \mathrm{Cu}(3) & \mathrm{P}(6) & \mathrm{C}(85) & \mathrm{C}(86) & 52.2(5) \\ \mathrm{C}(66) & \mathrm{P}(6) & \mathrm{C}(85) & \mathrm{C}(86) & -61.6(5) \\ \mathrm{C}(79) & \mathrm{P}(6) & \mathrm{C}(85) & \mathrm{C}(86) & -169.8(4) \\ \mathrm{Cu}(4) & \mathrm{P}(7) & \mathrm{C}(91) & \mathrm{C}(92) & 153.9(4) \\ \mathrm{C}(97) & \mathrm{P}(7) & \mathrm{C}(91) & \mathrm{C}(92) & 23.2(5) \\ \mathrm{C}(103) & \mathrm{P}(7) & \mathrm{C}(91) & \mathrm{C}(92) & -85.9(5) \\ \mathrm{Cu}(4) & \mathrm{P}(7) & \mathrm{C}(97) & \mathrm{C}(98) & -63.7(5) \\ \mathrm{C}(91) & \mathrm{P}(7) & \mathrm{C}(97) & \mathrm{C}(98) & 52.0(5) \\ \mathrm{C}(103) & \mathrm{P}(7) & \mathrm{C}(97) & \mathrm{C}(98) & 159.0(4) \\ \mathrm{Cu}(4) & \mathrm{P}(7) & \mathrm{C}(103) & \mathrm{C}(104) & 156.2(5) \\ \mathrm{C}(91) & \mathrm{P}(7) & \mathrm{C}(103) & \mathrm{C}(104) & 47.5(6) \\ \mathrm{C}(97) & \mathrm{P}(7) & \mathrm{C}(103) & \mathrm{C}(104) & -62.5(6) \\ & & & & \end{array}$

Table 8. Torsion angles $\left(^{0}\right)$-- continued

$\begin{array}{lllll}\mathrm{C}(73) & \mathrm{P}(5) & \mathrm{C}(67) & \mathrm{C}(72) & 98.2(5) \\ \mathrm{Cu}(3) & \mathrm{P}(5) & \mathrm{C}(73) & \mathrm{C}(78) & -44.4(7) \\ \mathrm{C}(61) & \mathrm{P}(5) & \mathrm{C}(73) & \mathrm{C}(78) & 71.4(6) \\ \mathrm{C}(67) & \mathrm{P}(5) & \mathrm{C}(73) & \mathrm{C}(78) & -179.7(5) \\ \mathrm{Cu}(3) & \mathrm{P}(6) & \mathrm{C}(66) & \mathrm{C}(65) & -161.2(4) \\ \mathrm{C}(79) & \mathrm{P}(6) & \mathrm{C}(66) & \mathrm{C}(65) & 73.4(5) \\ \mathrm{C}(85) & \mathrm{P}(6) & \mathrm{C}(66) & \mathrm{C}(65) & -35.7(5) \\ \mathrm{Cu}(3) & \mathrm{P}(6) & \mathrm{C}(79) & \mathrm{C}(84) & 12.6(6) \\ \mathrm{C}(66) & \mathrm{P}(6) & \mathrm{C}(79) & \mathrm{C}(84) & 125.8(5) \\ \mathrm{C}(85) & \mathrm{P}(6) & \mathrm{C}(79) & \mathrm{C}(84) & -125.2(5) \\ \mathrm{Cu}(3) & \mathrm{P}(6) & \mathrm{C}(85) & \mathrm{C}(90) & -120.2(5) \\ \mathrm{C}(66) & \mathrm{P}(6) & \mathrm{C}(85) & \mathrm{C}(90) & 125.9(5) \\ \mathrm{C}(79) & \mathrm{P}(6) & \mathrm{C}(85) & \mathrm{C}(90) & 17.8(6) \\ \mathrm{Cu}(4) & \mathrm{P}(7) & \mathrm{C}(91) & \mathrm{C}(96) & -27.5(4) \\ \mathrm{C}(97) & \mathrm{P}(7) & \mathrm{C}(91) & \mathrm{C}(96) & -158.2(4) \\ \mathrm{C}(103) & \mathrm{P}(7) & \mathrm{C}(91) & \mathrm{C}(96) & 92.6(4) \\ \mathrm{Cu}(4) & \mathrm{P}(7) & \mathrm{C}(97) & \mathrm{C}(102) & 106.7(5) \\ \mathrm{C}(91) & \mathrm{P}(7) & \mathrm{C}(97) & \mathrm{C}(102) & -137.6(4) \\ \mathrm{C}(103) & \mathrm{P}(7) & \mathrm{C}(97) & \mathrm{C}(102) & -30.6(5) \\ \mathrm{Cu}(4) & \mathrm{P}(7) & \mathrm{C}(103) & \mathrm{C}(108) & -17.8(6) \\ \mathrm{C}(91) & \mathrm{P}(7) & \mathrm{C}(103) & \mathrm{C}(108) & -126.6(5) \\ \mathrm{C}(97) & \mathrm{P}(7) & \mathrm{C}(103) & \mathrm{C}(108) & 123.4(5)\end{array}$

$\begin{array}{lllll}\text { atom1 } & \text { atom2 } & \text { atom3 } & \text { atom4 } & \text { angle } \\ \mathrm{Cu}(4) & \mathrm{P}(8) & \mathrm{C}(96) & \mathrm{C}(95) & -156.4(5) \\ \mathrm{C}(109) & \mathrm{P}(8) & \mathrm{C}(96) & \mathrm{C}(95) & 83.9(5) \\ \mathrm{C}(115) & \mathrm{P}(8) & \mathrm{C}(96) & \mathrm{C}(95) & -24.8(5) \\ \mathrm{Cu}(4) & \mathrm{P}(8) & \mathrm{C}(109) & \mathrm{C}(114) & 57.6(6) \\ \mathrm{C}(96) & \mathrm{P}(8) & \mathrm{C}(109) & \mathrm{C}(114) & 167.1(5) \\ \mathrm{C}(115) & \mathrm{P}(8) & \mathrm{C}(109) & \mathrm{C}(114) & -83.8(5) \\ \mathrm{Cu}(4) & \mathrm{P}(8) & \mathrm{C}(115) & \mathrm{C}(120) & 22.2(7) \\ \mathrm{C}(96) & \mathrm{P}(8) & \mathrm{C}(115) & \mathrm{C}(120) & -93.5(5) \\ \mathrm{C}(109) & \mathrm{P}(8) & \mathrm{C}(115) & \mathrm{C}(120) & 158.5(5) \\ \mathrm{C}(6) & \mathrm{C}(1) & \mathrm{C}(2) & \mathrm{C}(3) & 1.6(9) \\ \mathrm{P}(2) & \mathrm{C}(1) & \mathrm{C}(6) & \mathrm{C}(5) & 178.8(5) \\ \mathrm{C}(2) & \mathrm{C}(1) & \mathrm{C}(6) & \mathrm{C}(5) & -2.2(8) \\ \mathrm{C}(2) & \mathrm{C}(3) & \mathrm{C}(4) & \mathrm{C}(5) & -0.2(8) \\ \mathrm{C}(4) & \mathrm{C}(5) & \mathrm{C}(6) & \mathrm{P}(1) & -179.5(5) \\ \mathrm{P}(1) & \mathrm{C}(7) & \mathrm{C}(8) & \mathrm{C}(9) & 177.7(8) \\ \mathrm{P}(1) & \mathrm{C}(7) & \mathrm{C}(12) & \mathrm{C}(11) & 179.5(6) \\ \mathrm{C}(7) & \mathrm{C}(8) & \mathrm{C}(9) & \mathrm{C}(10) & 4(1) \\ \mathrm{C}(9) & \mathrm{C}(10) & \mathrm{C}(11) & \mathrm{C}(12) & 0(1) \\ \mathrm{P}(1) & \mathrm{C}(13) & \mathrm{C}(14) & \mathrm{C}(15) & 179.4(6) \\ \mathrm{P}(1) & \mathrm{C}(13) & \mathrm{C}(18) & \mathrm{C}(17) & -179.4(6) \\ \mathrm{C}(13) & \mathrm{C}(14) & \mathrm{C}(15) & \mathrm{C}(16) & 2(1) \\ \mathrm{C}(15) & \mathrm{C}(16) & \mathrm{C}(17) & \mathrm{C}(18) & 0(1) \\ \mathrm{P}(2) & \mathrm{C}(19) & \mathrm{C}(20) & \mathrm{C}(21) & 178.1(7) \\ \mathrm{P}(2) & \mathrm{C}(19) & \mathrm{C}(24) & \mathrm{C}(23) & -176.5(7) \\ \mathrm{C}(19) & \mathrm{C}(20) & \mathrm{C}(21) & \mathrm{C}(22) & -1(1) \\ & & & & \end{array}$




$\begin{array}{lllll}\mathrm{C}(20) & \mathrm{C}(21) & \mathrm{C}(22) & \mathrm{C}(23) & 0(1) \\ \mathrm{C}(22) & \mathrm{C}(23) & \mathrm{C}(24) & \mathrm{C}(19) & -1(1) \\ \mathrm{C}(30) & \mathrm{C}(25) & \mathrm{C}(26) & \mathrm{C}(27) & 0.4(8) \\ \mathrm{C}(26) & \mathrm{C}(25) & \mathrm{C}(30) & \mathrm{C}(29) & 0.5(9) \\ \mathrm{C}(26) & \mathrm{C}(27) & \mathrm{C}(28) & \mathrm{C}(29) & 0(1) \\ \mathrm{C}(28) & \mathrm{C}(29) & \mathrm{C}(30) & \mathrm{C}(25) & 0(1) \\ \mathrm{C}(36) & \mathrm{C}(31) & \mathrm{C}(32) & \mathrm{C}(33) & 1.3(8) \\ \mathrm{P}(3) & \mathrm{C}(31) & \mathrm{C}(36) & \mathrm{C}(35) & -175.5(5) \\ \mathrm{C}(32) & \mathrm{C}(31) & \mathrm{C}(36) & \mathrm{C}(35) & 1.8(8) \\ \mathrm{C}(32) & \mathrm{C}(33) & \mathrm{C}(34) & \mathrm{C}(35) & 2.8(9) \\ \mathrm{C}(34) & \mathrm{C}(35) & \mathrm{C}(36) & \mathrm{P}(4) & 177.3(4) \\ \mathrm{P}(3) & \mathrm{C}(37) & \mathrm{C}(38) & \mathrm{C}(39) & -175.3(7) \\ & & & & \end{array}$

Table 8. Torsion angles $\left(^{\circ}\right)$-- continued

$\begin{array}{lllll}\mathrm{C}(21) & \mathrm{C}(22) & \mathrm{C}(23) & \mathrm{C}(24) & 1(1) \\ \mathrm{P}(2) & \mathrm{C}(25) & \mathrm{C}(26) & \mathrm{C}(27) & -175.5(6) \\ \mathrm{P}(2) & \mathrm{C}(25) & \mathrm{C}(30) & \mathrm{C}(29) & 176.3(6) \\ \mathrm{C}(25) & \mathrm{C}(26) & \mathrm{C}(27) & \mathrm{C}(28) & -1(1) \\ \mathrm{C}(27) & \mathrm{C}(28) & \mathrm{C}(29) & \mathrm{C}(30) & 0(1) \\ \mathrm{P}(3) & \mathrm{C}(31) & \mathrm{C}(32) & \mathrm{C}(33) & 178.4(5) \\ \mathrm{P}(3) & \mathrm{C}(31) & \mathrm{C}(36) & \mathrm{P}(4) & 4.7(7) \\ \mathrm{C}(32) & \mathrm{C}(31) & \mathrm{C}(36) & \mathrm{P}(4) & -178.0(4) \\ \mathrm{C}(31) & \mathrm{C}(32) & \mathrm{C}(33) & \mathrm{C}(34) & -3.6(8) \\ \mathrm{C}(33) & \mathrm{C}(34) & \mathrm{C}(35) & \mathrm{C}(36) & 0.2(6) \\ \mathrm{C}(34) & \mathrm{C}(35) & \mathrm{C}(36) & \mathrm{C}(31) & -2.6(8) \\ \mathrm{C}(42) & \mathrm{C}(37) & \mathrm{C}(38) & \mathrm{C}(39) & 0(1) \\ & & & & \end{array}$




$\begin{array}{llllllllll}\mathrm{C}(86) & \mathrm{C}(87) & \mathrm{C}(88) & \mathrm{C}(89) & -1(1) & \mathrm{C}(87) & \mathrm{C}(88) & \mathrm{C}(89) & \mathrm{C}(90) & 0.3(9) \\ \mathrm{C}(88) & \mathrm{C}(89) & \mathrm{C}(90) & \mathrm{C}(85) & 0.3(9) & \mathrm{P}(7) & \mathrm{C}(91) & \mathrm{C}(92) & \mathrm{C}(93) & 177.1(5)\end{array}$

Table 8. Torsion angles $\left(^{(}\right)$-- continued

$\begin{array}{llllllllll}\text { atom1 } & \text { atom2 } & \text { atom3 } & \text { atom4 } & \text { angle } & \text { atom1 } & \text { atom2 } & \text { atom3 } & \text { atom4 } & \text { angle } \\ \mathrm{C}(96) & \mathrm{C}(91) & \mathrm{C}(92) & \mathrm{C}(93) & -1.4(9) & \mathrm{P}(7) & \mathrm{C}(91) & \mathrm{C}(96) & \mathrm{P}(8) & 1.8(6) \\ \mathrm{P}(7) & \mathrm{C}(91) & \mathrm{C}(96) & \mathrm{C}(95) & -176.9(5) & \mathrm{C}(92) & \mathrm{C}(91) & \mathrm{C}(96) & \mathrm{P}(8) & -179.5(4) \\ \mathrm{C}(92) & \mathrm{C}(91) & \mathrm{C}(96) & \mathrm{C}(95) & 1.7(8) & \mathrm{C}(91) & \mathrm{C}(92) & \mathrm{C}(93) & \mathrm{C}(94) & 0.0(9) \\ \mathrm{C}(92) & \mathrm{C}(93) & \mathrm{C}(94) & \mathrm{C}(95) & 1(1) & \mathrm{C}(93) & \mathrm{C}(94) & \mathrm{C}(95) & \mathrm{C}(96) & -0.7(9) \\ \mathrm{C}(94) & \mathrm{C}(95) & \mathrm{C}(96) & \mathrm{P}(8) & -179.3(4) & \mathrm{C}(94) & \mathrm{C}(95) & \mathrm{C}(96) & \mathrm{C}(91) & -0.7(8) \\ \mathrm{P}(7) & \mathrm{C}(97) & \mathrm{C}(98) & \mathrm{C}(99) & 171.2(5) & \mathrm{C}(102) & \mathrm{C}(97) & \mathrm{C}(98) & \mathrm{C}(99) & 0.4(7) \\ \mathrm{P}(7) & \mathrm{C}(97) & \mathrm{C}(102) & \mathrm{C}(101) & -170.0(6) & \mathrm{C}(98) & \mathrm{C}(97) & \mathrm{C}(102) & \mathrm{C}(101) & 0.9(9) \\ \mathrm{C}(97) & \mathrm{C}(98) & \mathrm{C}(99) & \mathrm{C}(100) & 0(1) & \mathrm{C}(98) & \mathrm{C}(99) & \mathrm{C}(100) & \mathrm{C}(101) & 0(1) \\ \mathrm{C}(99) & \mathrm{C}(100) & \mathrm{C}(101) & \mathrm{C}(102) & 1(1) & \mathrm{C}(100) & \mathrm{C}(101) & \mathrm{C}(102) & \mathrm{C}(97) & -1(1) \\ \mathrm{P}(7) & \mathrm{C}(103) & \mathrm{C}(104) & \mathrm{C}(105) & -173.4(7) & \mathrm{C}(108) & \mathrm{C}(103) & \mathrm{C}(104) & \mathrm{C}(105) & 0(1) \\ \mathrm{P}(7) & \mathrm{C}(103) & \mathrm{C}(108) & \mathrm{C}(107) & 172.9(6) & \mathrm{C}(104) & \mathrm{C}(103) & \mathrm{C}(108) & \mathrm{C}(107) & -1(1) \\ \mathrm{C}(103) & \mathrm{C}(104) & \mathrm{C}(105) & \mathrm{C}(106) & 0(1) & \mathrm{C}(104) & \mathrm{C}(105) & \mathrm{C}(106) & \mathrm{C}(107) & 0(1) \\ \mathrm{C}(105) & \mathrm{C}(106) & \mathrm{C}(107) & \mathrm{C}(108) & -1(1) & \mathrm{C}(106) & \mathrm{C}(107) & \mathrm{C}(108) & \mathrm{C}(103) & 1(1) \\ \mathrm{P}(8) & \mathrm{C}(109) & \mathrm{C}(110) & \mathrm{C}(111) & 179.3(7) & \mathrm{C}(114) & \mathrm{C}(109) & \mathrm{C}(110) & \mathrm{C}(111) & 2(1) \\ \mathrm{P}(8) & \mathrm{C}(109) & \mathrm{C}(114) & \mathrm{C}(113) & -178.1(6) & \mathrm{C}(110) & \mathrm{C}(109) & \mathrm{C}(114) & \mathrm{C}(113) & 0(1) \\ \mathrm{C}(109) & \mathrm{C}(110) & \mathrm{C}(111) & \mathrm{C}(112) & -3(1) & \mathrm{C}(110) & \mathrm{C}(111) & \mathrm{C}(112) & \mathrm{C}(113) & 3(1) \\ \mathrm{C}(111) & \mathrm{C}(112) & \mathrm{C}(113) & \mathrm{C}(114) & -2(1) & \mathrm{C}(112) & \mathrm{C}(113) & \mathrm{C}(114) & \mathrm{C}(109) & 0(1) \\ \mathrm{P}(8) & \mathrm{C}(115) & \mathrm{C}(116) & \mathrm{C}(117) & 178.5(7) & \mathrm{C}(120) & \mathrm{C}(115) & \mathrm{C}(116) & \mathrm{C}(117) & 0(1) \\ \mathrm{P}(8) & \mathrm{C}(115) & \mathrm{C}(120) & \mathrm{C}(119) & -178.0(6) & \mathrm{C}(116) & \mathrm{C}(115) & \mathrm{C}(120) & \mathrm{C}(119) & 1(1) \\ \mathrm{C}(115) & \mathrm{C}(116) & \mathrm{C}(117) & \mathrm{C}(118) & 1(1) & \mathrm{C}(116) & \mathrm{C}(117) & \mathrm{C}(118) & \mathrm{C}(119) & -2(1) \\ \mathrm{C}(117) & \mathrm{C}(118) & \mathrm{C}(119) & \mathrm{C}(120) & 3(1) & \mathrm{C}(118) & \mathrm{C}(119) & \mathrm{C}(120) & \mathrm{C}(115) & -2(1)\end{array}$

The sign is positive if when looking from atom 2 to atom 3 a clock-wise motion of atom 1 would superimpose it on atom 4 . 


\title{
$[\mathrm{Cu}(\mu \mathrm{Br}) \mathrm{dppb}]_{2}(\mathbf{2})$
}

\section{Table S4.}

\author{
EXPERIMENTAL DETAILS
}

\author{
A. Crystal Data
}

\section{Empirical Formula \\ Formula Weight \\ Crystal Color, Habit \\ Crystal Dimensions \\ Crystal System \\ Lattice Type \\ Indexing Images \\ Detector Position \\ Pixel Size \\ Lattice Parameters}

Space Group

$Z$ value

$D_{\text {calc }}$

$\mathrm{F}_{000}$

$\mu(\mathrm{MoK} \alpha)$

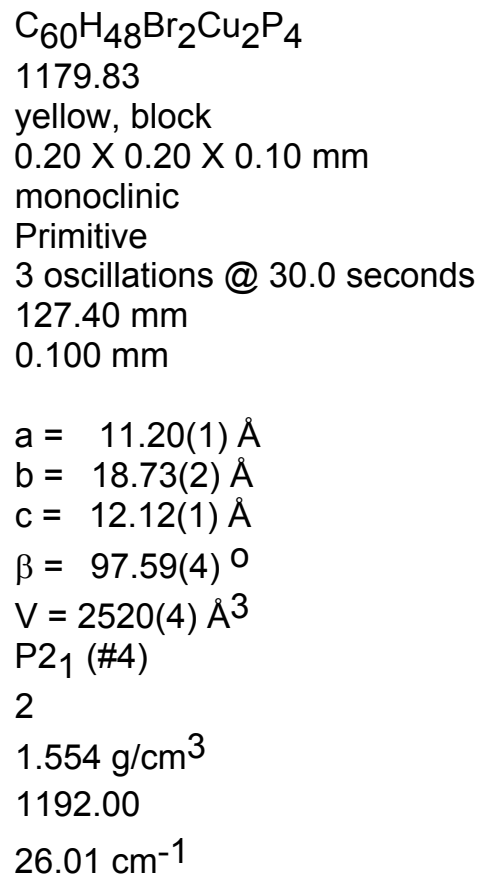

B. Intensity Measurements

Diffractometer

Radiation

Detector Aperture

Data Images

$\omega$ oscillation Range ( $\chi=45.0, \phi=30.0)$

Exposure Rate

$\omega$ oscillation Range ( $\chi=45.0, \phi=180.0)$

Exposure Rate

Detector Position

Pixel Size

$2 \theta$ max
Rigaku RAXIS-RAPID

$\operatorname{MoK} \alpha(\lambda=0.71075 \AA)$

graphite monochromated

$280 \mathrm{~mm} \times 256 \mathrm{~mm}$

44 exposures

$130.0-190.0^{\circ}$

$10.2 \mathrm{sec} . / \mathrm{O}$

$0.0-160.0^{\circ}$

$10.2 \mathrm{sec} . / \mathrm{O}$

$127.40 \mathrm{~mm}$

$0.100 \mathrm{~mm}$

$54.9^{\circ}$ 
No. of Reflections Measured

Total: 24210

Corrections

Unique: $24182\left(R_{\text {int }}=0.082\right)$

Lorentz-polarization

Absorption

(trans. factors: $0.5955-1.0000$ )

C. Structure Solution and Refinement

Structure Solution

Refinement

Function Minimized

Least Squares Weights

$2 \theta$ max cutoff

Anomalous Dispersion

No. Observations (All reflections)

No. Variables

Reflection/Parameter Ratio

Residuals: $R$ (All reflections)

Residuals: R1 $(\mid>2.00 \sigma(I))$

Residuals: wR2 (All reflections)

Goodness of Fit Indicator

Max Shift/Error in Final Cycle

Maximum peak in Final Diff. Map

Minimum peak in Final Diff. Map
Direct Methods (SIR92)

Full-matrix least-squares on $\mathrm{F}^{2}$

$\Sigma \mathrm{w}\left(\mathrm{Fo}^{2}-\mathrm{Fc}^{2}\right)^{2}$

$1 /\left[0.0003 \mathrm{Fo}^{2}+0.2000 \sigma\left(\mathrm{Fo}^{2}\right)\right] /\left(4 \mathrm{Fo}^{2}\right)$

$54.9^{\circ}$

All non-hydrogen atoms

24182

661

36.58

0.069

0.045

0.109

1.396

0.000

$3.08 e^{-} / \AA^{3}$

$-2.46 e^{-} / \AA^{3}$

Table 4. Bond lengths $(\AA)$

$\begin{array}{llllll}\text { atom } & \text { atom } & \text { distance } & \text { atom } & \text { atom } & \text { distance } \\ \mathrm{Br}(1) & \mathrm{Cu}(1) & 2.4536(6) & \mathrm{Br}(1) & \mathrm{Cu}(2) & 2.4948(6) \\ \mathrm{Br}(2) & \mathrm{Cu}(1) & 2.5328(6) & \mathrm{Br}(2) & \mathrm{Cu}(2) & 2.4290(7) \\ \mathrm{Cu}(1) & \mathrm{P}(1) & 2.262(1) & \mathrm{Cu}(1) & \mathrm{P}(2) & 2.257(1) \\ \mathrm{Cu}(2) & \mathrm{P}(3) & 2.259(1) & \mathrm{Cu}(2) & \mathrm{P}(4) & 2.259(1) \\ \mathrm{P}(1) & \mathrm{C}(1) & 1.839(4) & \mathrm{P}(1) & \mathrm{C}(7) & 1.825(4) \\ \mathrm{P}(1) & \mathrm{C}(13) & 1.824(4) & \mathrm{P}(2) & \mathrm{C}(6) & 1.856(5) \\ \mathrm{P}(2) & \mathrm{C}(19) & 1.828(4) & \mathrm{P}(2) & \mathrm{C}(25) & 1.832(4) \\ \mathrm{P}(3) & \mathrm{C}(31) & 1.849(4) & \mathrm{P}(3) & \mathrm{C}(37) & 1.824(5) \\ \mathrm{P}(3) & \mathrm{C}(43) & 1.830(4) & \mathrm{P}(4) & \mathrm{C}(36) & 1.822(5) \\ \mathrm{P}(4) & \mathrm{C}(49) & 1.821(4) & \mathrm{P}(4) & \mathrm{C}(55) & 1.827(4) \\ \mathrm{C}(1) & \mathrm{C}(2) & 1.401(6) & \mathrm{C}(1) & \mathrm{C}(6) & 1.417(5) \\ \mathrm{C}(2) & \mathrm{C}(3) & 1.384(6) & \mathrm{C}(3) & \mathrm{C}(2) & 1.384(6) \\ \mathrm{C}(3) & \mathrm{C}(4) & 1.388(6) & \mathrm{C}(4) & \mathrm{C}(3) & 1.388(6) \\ \mathrm{C}(4) & \mathrm{C}(5) & 1.378(6) & \mathrm{C}(5) & \mathrm{C}(6) & 1.388(6) \\ \mathrm{C}(7) & \mathrm{C}(8) & 1.408(5) & \mathrm{C}(7) & \mathrm{C}(12) & 1.371(6) \\ \mathrm{C}(8) & \mathrm{C}(9) & 1.386(5) & \mathrm{C}(9) & \mathrm{C}(10) & 1.371(7) \\ \mathrm{C}(10) & \mathrm{C}(11) & 1.395(6) & \mathrm{C}(11) & \mathrm{C}(12) & 1.408(5) \\ \mathrm{C}(13) & \mathrm{C}(14) & 1.402(6) & \mathrm{C}(13) & \mathrm{C}(18) & 1.386(6)\end{array}$




\begin{tabular}{|c|c|c|c|c|c|c|c|}
\hline$C(14)$ & \multicolumn{2}{|l|}{$C(15)$} & $1.379(6)$ & \multicolumn{2}{|l|}{$C(15)$} & $C(16)$ & $1.383(6)$ \\
\hline$C(16)$ & \multicolumn{2}{|l|}{$C(17)$} & $1.385(7)$ & \multicolumn{2}{|l|}{$\mathrm{C}(17)$} & $C(18)$ & $1.382(7)$ \\
\hline$C(19)$ & \multicolumn{2}{|l|}{$C(20)$} & $1.370(7)$ & \multicolumn{2}{|l|}{$C(19)$} & $C(24)$ & 1.397(6) \\
\hline$C(20)$ & \multicolumn{2}{|l|}{$C(21)$} & $1.391(6)$ & \multicolumn{2}{|l|}{$C(21)$} & $C(22)$ & $1.381(7)$ \\
\hline$C(22)$ & \multicolumn{2}{|l|}{$C(23)$} & $1.362(8)$ & \multicolumn{2}{|l|}{$\mathrm{C}(23)$} & $C(24)$ & $1.384(7)$ \\
\hline$C(25)$ & \multicolumn{2}{|l|}{$C(26)$} & $1.386(6)$ & \multicolumn{2}{|l|}{$C(25)$} & $C(30)$ & $1.386(6)$ \\
\hline$C(26)$ & \multicolumn{2}{|l|}{$C(27)$} & 1.398(6) & $C(27)$ & & $C(28)$ & $1.375(7)$ \\
\hline$C(28)$ & $C(29)$ & & $1.384(7)$ & $C(29)$ & & $C(30)$ & $1.379(6)$ \\
\hline$C(31)$ & $C(32)$ & & $1.383(6)$ & $C(31)$ & & $C(36)$ & $1.434(6)$ \\
\hline$C(32)$ & $C(33)$ & & $1.386(7)$ & $C(33)$ & & C(34) & $1.389(7)$ \\
\hline$C(34)$ & $C(35)$ & & $1.390(7)$ & $C(35)$ & & $C(36)$ & $1.384(6)$ \\
\hline$C(37)$ & $C(38)$ & & $1.392(6)$ & $C(37)$ & & $C(42)$ & $1.389(6)$ \\
\hline$C(38)$ & $C(39)$ & & $1.400(7)$ & C(39) & & $C(40)$ & $1.380(7)$ \\
\hline$C(40)$ & $C(41)$ & & $1.372(7)$ & $\mathrm{C}(41)$ & & $C(42)$ & $1.368(7)$ \\
\hline$C(43)$ & C(44) & & $1.377(7)$ & $C(43)$ & & $C(48)$ & $1.407(6)$ \\
\hline$C(44)$ & $C(45)$ & & $1.393(6)$ & $C(45)$ & & $C(46)$ & $1.386(7)$ \\
\hline$C(46)$ & $C(47)$ & & $1.372(8)$ & $C(47)$ & & $C(48)$ & $1.400(6)$ \\
\hline$C(49)$ & $C(50)$ & & $1.387(6)$ & C(49) & & $C(54)$ & $1.396(6)$ \\
\hline$C(50)$ & $C(51)$ & & $1.379(6)$ & $C(51)$ & & $C(52)$ & $1.384(6)$ \\
\hline Table 4 & 3ond leng & $s(\AA)$ & -- continued & & & & \\
\hline atom & atom & & distance & atom & & atom & distance \\
\hline$C(52)$ & $C(53)$ & & $1.386(6)$ & $C(53)$ & & $C(54)$ & $1.387(6)$ \\
\hline$C(55)$ & $C(56)$ & & $1.403(6)$ & $C(55)$ & & $C(60)$ & $1.386(7)$ \\
\hline$C(56)$ & $C(57)$ & & $1.383(6)$ & C(57) & & C(58) & $1.377(8)$ \\
\hline$C(58)$ & $C(59)$ & & $1.380(7)$ & $C(59)$ & & $C(60)$ & $1.378(6)$ \\
\hline Table 6 & 3ond ang & $(0)$ & & & & & \\
\hline atom & atom & atom & angle & atom & atom & atom & angle \\
\hline $\mathrm{Cu}(2)$ & $\operatorname{Br}(1)$ & $\mathrm{Cu}(1)$ & 69.96(2) & $\operatorname{Br}(1)$ & $\mathrm{Cu}(1)$ & $\operatorname{Br}(2)$ & $106.61(2)$ \\
\hline $\operatorname{Br}(1)$ & $\mathrm{Cu}(1)$ & $P(1)^{\prime}$ & $121.14(3)$ & $\operatorname{Br}(1)$ & $\mathrm{Cu}(1)$ & $P(2)$ & $120.62(4)$ \\
\hline $\operatorname{Br}(1)$ & $\mathrm{Cu}(2)$ & $\operatorname{Br}(2)$ & $108.59(2)$ & $\operatorname{Br}(1)$ & $\mathrm{Cu}(2)$ & $P(3)$ & $108.99(4)$ \\
\hline $\operatorname{Br}(1)$ & $\mathrm{Cu}(2)$ & $P(4)^{\prime}$ & 110.41(4) & $\mathrm{Cu}(2)$ & $\operatorname{Br}(2)$ & $\mathrm{Cu}(1)$ & $69.71(2)$ \\
\hline $\operatorname{Br}(2)$ & $\mathrm{Cu}(1)$ & $P(1)$ & $109.79(3)$ & $\operatorname{Br}(2)$ & $\mathrm{Cu}(1)$ & $P(2)$ & $106.80(3)$ \\
\hline $\mathrm{Br}(2)$ & $\mathrm{Cu}(2)$ & $P(3)$ & $121.35(4)$ & $\operatorname{Br}(2)$ & $\mathrm{Cu}(2)$ & $P(4)$ & $118.14(4)$ \\
\hline$P(2)$ & $\mathrm{Cu}(1)$ & $P(1)$ & $90.59(4)$ & $\mathrm{Cu}(1)$ & $P(1)$ & $C(1)$ & $102.2(1)$ \\
\hline $\mathrm{Cu}(1)$ & $P(1)$ & $C(7)$ & 116.3(1) & $\mathrm{Cu}(1)$ & $P(1)$ & $C(13)$ & $123.2(1)$ \\
\hline $\mathrm{Cu}(1)$ & $P(2)$ & $C(6)$ & $102.3(1)$ & $\mathrm{Cu}(1)$ & $P(2)$ & C(19) & 119.6(1) \\
\hline $\mathrm{Cu}(1)$ & $P(2)$ & $C(25)$ & 121.2(1) & $P(4)^{\prime}$ & $\mathrm{Cu}(2)$ & $P(3)$ & $87.82(4)$ \\
\hline $\mathrm{Cu}(2)$ & $P(3)$ & $C(31)$ & 101.0(1) & $\mathrm{Cu}(2)$ & $P(3)$ & $C(43)$ & $114.8(2)$ \\
\hline $\mathrm{Cu}(2)$ & $P(3)$ & $C(37)$ & $125.7(1)$ & $\mathrm{Cu}(2)$ & $P(4)$ & $C(36)$ & $101.6(1)$ \\
\hline $\mathrm{Cu}(2)$ & $\mathrm{P}(4)$ & C(55) & $113.8(1)$ & $\mathrm{Cu}(2)$ & $P(4)$ & C(49) & 124.7(1) \\
\hline$P(1)$ & $C(1)$ & $C(6)$ & $119.8(3)$ & $C(7)$ & $P(1)$ & $C(1)$ & 105.1(2) \\
\hline$P(1)$ & $C(1)$ & $C(2)$ & $122.1(3)$ & $C(13)$ & $P(1)$ & $\mathrm{C}(1)$ & $104.2(2)$ \\
\hline$P(1)$ & $C(7)$ & $C(8)$ & $123.8(3)$ & $P(1)$ & $C(7)$ & $C(12)$ & 117.5(3) \\
\hline$C(13)$ & $P(1)$ & $\mathrm{C}(7)$ & $103.8(2)$ & $P(1)$ & $C(13)$ & $C(14)$ & $117.0(3)$ \\
\hline$P(1)$ & $C(13)$ & $C(18)$ & $125.1(3)$ & $P(2)$ & $C(6)$ & $C(1)$ & $118.4(3)$ \\
\hline$C(19)$ & $P(2)$ & $C(6)$ & $102.9(2)$ & $C(25)$ & $P(2)$ & $C(6)$ & $103.6(2)$ \\
\hline
\end{tabular}




$\begin{array}{llllllll}\mathrm{P}(2) & \mathrm{C}(6) & \mathrm{C}(5) & 121.9(3) & \mathrm{C}(25) & \mathrm{P}(2) & \mathrm{C}(19) & 104.6(2) \\ \mathrm{P}(2) & \mathrm{C}(19) & \mathrm{C}(20) & 120.1(3) & \mathrm{P}(2) & \mathrm{C}(19) & \mathrm{C}(24) & 121.4(4) \\ \mathrm{P}(2) & \mathrm{C}(25) & \mathrm{C}(26) & 122.9(3) & \mathrm{P}(2) & \mathrm{C}(25) & \mathrm{C}(30) & 118.5(3) \\ \mathrm{P}(3) & \mathrm{C}(31) & \mathrm{C}(36) & 116.0(3) & \mathrm{C}(43) & \mathrm{P}(3) & \mathrm{C}(31) & 101.3(2) \\ \mathrm{P}(3) & \mathrm{C}(31) & \mathrm{C}(32) & 124.1(3) & \mathrm{C}(37) & \mathrm{P}(3) & \mathrm{C}(31) & 104.4(2) \\ \mathrm{C}(43) & \mathrm{P}(3) & \mathrm{C}(37) & 106.2(2) & \mathrm{P}(3) & \mathrm{C}(37) & \mathrm{C}(38) & 122.4(3) \\ \mathrm{P}(3) & \mathrm{C}(37) & \mathrm{C}(42) & 118.6(3) & \mathrm{P}(3) & \mathrm{C}(43) & \mathrm{C}(44) & 118.8(3) \\ \mathrm{P}(3) & \mathrm{C}(43) & \mathrm{C}(48) & 121.0(4) & \mathrm{P}(4) & \mathrm{C}(36) & \mathrm{C}(31) & 119.0(3) \\ \mathrm{C}(55) & \mathrm{P}(4) & \mathrm{C}(36) & 104.4(2) & \mathrm{P}(4) & \mathrm{C}(36) & \mathrm{C}(35) & 122.9(3) \\ \mathrm{C}(49) & \mathrm{P}(4) & \mathrm{C}(36) & 104.6(2) & \mathrm{C}(55) & \mathrm{P}(4) & \mathrm{C}(49) & 105.4(2) \\ \mathrm{P}(4) & \mathrm{C}(49) & \mathrm{C}(50) & 124.2(3) & \mathrm{P}(4) & \mathrm{C}(49) & \mathrm{C}(54) & 117.4(3) \\ \mathrm{P}(4) & \mathrm{C}(55) & \mathrm{C}(56) & 123.2(4) & \mathrm{P}(4) & \mathrm{C}(55) & \mathrm{C}(60) & 118.0(3) \\ \mathrm{C}(6) & \mathrm{C}(1) & \mathrm{C}(2) & 118.2(4) & \mathrm{C}(1) & \mathrm{C}(2) & \mathrm{C}(3) & 121.4(4) \\ \mathrm{C}(1) & \mathrm{C}(6) & \mathrm{C}(5) & 119.7(4) & \mathrm{C}(2) & \mathrm{C}(3) & \mathrm{C}(4) & 119.4(4) \\ \mathrm{C}(3) & \mathrm{C}(4) & \mathrm{C}(5) & 120.4(4) & \mathrm{C}(4) & \mathrm{C}(5) & \mathrm{C}(6) & 120.8(4) \\ \mathrm{C}(12) & \mathrm{C}(7) & \mathrm{C}(8) & 118.7(3) & \mathrm{C}(7) & \mathrm{C}(8) & \mathrm{C}(9) & 120.1(4) \\ \mathrm{C}(7) & \mathrm{C}(12) & \mathrm{C}(11) & 121.6(4) & \mathrm{C}(8) & \mathrm{C}(9) & \mathrm{C}(10) & 120.7(4) \\ \mathrm{C}(9) & \mathrm{C}(10) & \mathrm{C}(11) & 120.4(4) & \mathrm{C}(10) & \mathrm{C}(11) & \mathrm{C}(12) & 118.5(4)\end{array}$

Table 6. Bond angles $\left(^{\circ}\right)$-- continued

$\begin{array}{llllllll}\text { atom } & \text { atom } & \text { atom } & \text { angle } & \text { atom } & \text { atom } & \text { atom } & \text { angle } \\ \mathrm{C}(18) & \mathrm{C}(13) & \mathrm{C}(14) & 117.9(4) & \mathrm{C}(13) & \mathrm{C}(14) & \mathrm{C}(15) & 120.8(4) \\ \mathrm{C}(13) & \mathrm{C}(18) & \mathrm{C}(17) & 120.9(4) & \mathrm{C}(14) & \mathrm{C}(15) & \mathrm{C}(16) & 120.9(4) \\ \mathrm{C}(15) & \mathrm{C}(16) & \mathrm{C}(17) & 118.5(4) & \mathrm{C}(16) & \mathrm{C}(17) & \mathrm{C}(18) & 120.9(4) \\ \mathrm{C}(24) & \mathrm{C}(19) & \mathrm{C}(20) & 118.4(4) & \mathrm{C}(19) & \mathrm{C}(20) & \mathrm{C}(21) & 121.7(4) \\ \mathrm{C}(19) & \mathrm{C}(24) & \mathrm{C}(23) & 119.6(5) & \mathrm{C}(20) & \mathrm{C}(21) & \mathrm{C}(22) & 119.2(5) \\ \mathrm{C}(21) & \mathrm{C}(22) & \mathrm{C}(23) & 119.7(4) & \mathrm{C}(22) & \mathrm{C}(23) & \mathrm{C}(24) & 121.4(4) \\ \mathrm{C}(30) & \mathrm{C}(25) & \mathrm{C}(26) & 118.4(4) & \mathrm{C}(25) & \mathrm{C}(26) & \mathrm{C}(27) & 120.4(4) \\ \mathrm{C}(25) & \mathrm{C}(30) & \mathrm{C}(29) & 121.4(4) & \mathrm{C}(26) & \mathrm{C}(27) & \mathrm{C}(28) & 120.1(4) \\ \mathrm{C}(27) & \mathrm{C}(28) & \mathrm{C}(29) & 119.8(4) & \mathrm{C}(28) & \mathrm{C}(29) & \mathrm{C}(30) & 119.8(5) \\ \mathrm{C}(36) & \mathrm{C}(31) & \mathrm{C}(32) & 119.9(4) & \mathrm{C}(31) & \mathrm{C}(32) & \mathrm{C}(33) & 120.4(4) \\ \mathrm{C}(31) & \mathrm{C}(36) & \mathrm{C}(35) & 118.1(4) & \mathrm{C}(32) & \mathrm{C}(33) & \mathrm{C}(34) & 120.4(4) \\ \mathrm{C}(33) & \mathrm{C}(34) & \mathrm{C}(35) & 119.5(4) & \mathrm{C}(34) & \mathrm{C}(35) & \mathrm{C}(36) & 121.6(4) \\ \mathrm{C}(42) & \mathrm{C}(37) & \mathrm{C}(38) & 118.6(4) & \mathrm{C}(37) & \mathrm{C}(38) & \mathrm{C}(39) & 119.9(4) \\ \mathrm{C}(37) & \mathrm{C}(42) & \mathrm{C}(41) & 120.7(4) & \mathrm{C}(38) & \mathrm{C}(39) & \mathrm{C}(40) & 120.3(4) \\ \mathrm{C}(39) & \mathrm{C}(40) & \mathrm{C}(41) & 119.1(4) & \mathrm{C}(40) & \mathrm{C}(41) & \mathrm{C}(42) & 121.4(5) \\ \mathrm{C}(48) & \mathrm{C}(43) & \mathrm{C}(44) & 119.7(4) & \mathrm{C}(43) & \mathrm{C}(44) & \mathrm{C}(45) & 120.8(4) \\ \mathrm{C}(43) & \mathrm{C}(48) & \mathrm{C}(47) & 118.7(5) & \mathrm{C}(44) & \mathrm{C}(45) & \mathrm{C}(46) & 119.7(5) \\ \mathrm{C}(45) & \mathrm{C}(46) & \mathrm{C}(47) & 119.9(4) & \mathrm{C}(46) & \mathrm{C}(47) & \mathrm{C}(48) & 121.2(4) \\ \mathrm{C}(54) & \mathrm{C}(49) & \mathrm{C}(50) & 118.2(4) & \mathrm{C}(49) & \mathrm{C}(50) & \mathrm{C}(51) & 121.3(4) \\ \mathrm{C}(49) & \mathrm{C}(54) & \mathrm{C}(53) & 120.5(4) & \mathrm{C}(50) & \mathrm{C}(51) & \mathrm{C}(52) & 120.3(4) \\ \mathrm{C}(51) & \mathrm{C}(52) & \mathrm{C}(53) & 119.2(4) & \mathrm{C}(52) & \mathrm{C}(53) & \mathrm{C}(54) & 120.4(4) \\ \mathrm{C}(60) & \mathrm{C}(55) & \mathrm{C}(56) & 118.6(4) & \mathrm{C}(55) & \mathrm{C}(56) & \mathrm{C}(57) & 119.7(5) \\ \mathrm{C}(55) & \mathrm{C}(60) & \mathrm{C}(59) & 120.8(4) & \mathrm{C}(56) & \mathrm{C}(57) & \mathrm{C}(58) & 121.0(4) \\ \mathrm{C}(57) & \mathrm{C}(58) & \mathrm{C}(59) & 119.3(4) & \mathrm{C}(58) & \mathrm{C}(59) & \mathrm{C}(60) & 120.5(5)\end{array}$

Table 8. Torsion Angles $\left({ }^{\circ}\right)$

atom1 atom2 atom3 atom4 angle atom1 atom2 atom3 atom4 angle 


\begin{tabular}{|c|c|c|c|c|c|c|c|c|c|}
\hline $\mathrm{Cu}(2)$ & $\operatorname{Br}(1)$ & $\mathrm{Cu}(1)$ & $\operatorname{Br}(2)$ & $-17.02(3)$ & $\mathrm{Cu}(2)$ & $\operatorname{Br}(1)$ & $\mathrm{Cu}(1)$ & $P(1)$ & $-143.39(4)$ \\
\hline $\mathrm{Cu}(2)$ & $\operatorname{Br}(1)$ & $\mathrm{Cu}(1)$ & $P(2)^{\prime}$ & $104.78(4)$ & $\mathrm{Cu}(1)$ & $\operatorname{Br}(1)$ & $\mathrm{Cu}(2)$ & $\operatorname{Br}(2)$ & $17.97(3)$ \\
\hline $\mathrm{Cu}(1)$ & $\operatorname{Br}(1)$ & $\mathrm{Cu}(2)$ & $P(3)$ & 152.09(4) & $\mathrm{Cu}(1)$ & $\operatorname{Br}(1)$ & $\mathrm{Cu}(2)$ & $P(4)$ & $-113.03(4)$ \\
\hline $\mathrm{Cu}(2)$ & $\operatorname{Br}(2)$ & $\mathrm{Cu}(1)$ & $\operatorname{Br}(1)$ & $17.52(3)$ & $\mathrm{Cu}(2)$ & $\operatorname{Br}(2)$ & $\mathrm{Cu}(1)$ & $P(1)$ & $150.44(4)$ \\
\hline $\mathrm{Cu}(2)$ & $\operatorname{Br}(2)$ & $\mathrm{Cu}(1)$ & $P(2)$ & $-112.66(4)$ & $\mathrm{Cu}(1)$ & $\operatorname{Br}(2)$ & $\mathrm{Cu}(2)$ & $\operatorname{Br}(1)$ & $-17.42(3)$ \\
\hline $\mathrm{Cu}(1)$ & $\operatorname{Br}(2)$ & $\mathrm{Cu}(2)$ & $P(3)$ & $-144.78(4)$ & $\mathrm{Cu}(1)$ & $\operatorname{Br}(2)$ & $\mathrm{Cu}(2)$ & $P(4)^{\prime}$ & $109.25(4)$ \\
\hline $\operatorname{Br}(1)$ & $\mathrm{Cu}(1)$ & $P(1)$ & $C(1)$ & $-149.0(1)$ & $\operatorname{Br}(1)$ & $\mathrm{Cu}(1)$ & $P(1)$ & $C(7)$ & $-35.2(2)$ \\
\hline $\operatorname{Br}(1)$ & $\mathrm{Cu}(1)$ & $P(1)$ & $C(13)$ & $94.8(2)$ & $\operatorname{Br}(2)$ & $\mathrm{Cu}(1)$ & $P(1)$ & $C(1)$ & $86.0(1)$ \\
\hline $\operatorname{Br}(2)$ & $\mathrm{Cu}(1)$ & $P(1)$ & $C(7)$ & $-160.1(1)$ & $\operatorname{Br}(2)$ & $\mathrm{Cu}(1)$ & $P(1)$ & $C(13)$ & $-30.1(2)$ \\
\hline$P(2)^{\prime}$ & $\mathrm{Cu}(1)$ & $P(1)$ & $C(1)$ & $-22.1(1)^{\prime}$ & $P(2)$ & $\mathrm{Cu}(1)$ & $P(1)$ & $C(7)$ & $91.8(1)$ \\
\hline$P(2)$ & $\mathrm{Cu}(1)$ & $P(1)$ & $C(13)$ & $-138.2(2)$ & $\operatorname{Br}(1)$ & $\mathrm{Cu}(1)$ & $P(2)$ & $\mathrm{C}(6)$ & 149.8(1) \\
\hline $\operatorname{Br}(1)$ & $\mathrm{Cu}(1)$ & $P(2)$ & $C(19)$ & $37.1(2)$ & $\operatorname{Br}(1)$ & $\mathrm{Cu}(1)$ & $P(2)$ & $C(25)$ & $-95.9(2)$ \\
\hline $\operatorname{Br}(2)$ & $\mathrm{Cu}(1)$ & $P(2)$ & $C(6)$ & $-88.5(1)$ & $\operatorname{Br}(2)$ & $\mathrm{Cu}(1)$ & $P(2)$ & $C(19)$ & $158.8(2)$ \\
\hline $\operatorname{Br}(2)$ & $\mathrm{Cu}(1)$ & $P(2)$ & $C(25)$ & $25.8(2)$ & $P(1)$ & $\mathrm{Cu}(1)$ & $P(2)$ & $C(6)$ & $22.4(1)$ \\
\hline$P(1)$ & $\mathrm{Cu}(1)$ & $P(2)$ & C(19) & $-90.3(2)$ & $P(1)$ & $\mathrm{Cu}(1)$ & $P(2)$ & $C(25)$ & $136.7(2)$ \\
\hline $\operatorname{Br}(1)$ & $\mathrm{Cu}(2)$ & $P(3)$ & $C(31)$ & $77.7(1)$ & $\operatorname{Br}(1)$ & $\mathrm{Cu}(2)$ & $P(3)$ & $C(37)$ & $-39.1(2)$ \\
\hline $\operatorname{Br}(1)$ & $\mathrm{Cu}(2)$ & $P(3)$ & $C(43)$ & $-174.3(2)$ & $\operatorname{Br}(2)$ & $\mathrm{Cu}(2)$ & $P(3)$ & $C(31)$ & $-155.1(1)$ \\
\hline $\operatorname{Br}(2)$ & $\mathrm{Cu}(2)$ & $P(3)$ & $C(37)$ & $88.1(2)$ & $\operatorname{Br}(2)$ & $\mathrm{Cu}(2)$ & $P(3)$ & $C(43)$ & $-47.1(2)$ \\
\hline$P(4)$ & $\mathrm{Cu}(2)$ & $P(3)$ & $C(31)$ & $-33.2(1)$ & $P(4)$ & $\mathrm{Cu}(2)$ & $P(3)$ & $C(37)$ & $-150.0(2)$ \\
\hline$P(4)$ & $\mathrm{Cu}(2)$ & $P(3)$ & $C(43)$ & $74.9(2)$ & $\operatorname{Br}(1)$ & $\mathrm{Cu}(2)$ & $P(4)$ & $C(36)$ & $-78.7(1)$ \\
\hline $\operatorname{Br}(1)$ & $\mathrm{Cu}(2)$ & $P(4)$ & C(49) & $38.4(2)$ & $\operatorname{Br}(1)$ & $\mathrm{Cu}(2)$ & $P(4)$ & $C(55)$ & $169.6(2)$ \\
\hline $\operatorname{Br}(2)$ & $\mathrm{Cu}(2)$ & $P(4)$ & $C(36)$ & $155.5(1)$ & $\operatorname{Br}(2)$ & $\mathrm{Cu}(2)$ & $P(4)$ & C(49) & $-87.4(2)$ \\
\hline $\operatorname{Br}(2)$ & $\mathrm{Cu}(2)$ & $P(4)$ & $C(55)$ & $43.8(2)$ & $P(3)$ & $\mathrm{Cu}(2)$ & $P(4)$ & $C(36)$ & $30.7(1)$ \\
\hline$P(3)$ & $\mathrm{Cu}(2)$ & $P(4)$ & C(49) & $147.8(2)$ & $P(3)$ & $\mathrm{Cu}(2)$ & $P(4)$ & $C(55)$ & $-80.9(2)$ \\
\hline $\mathrm{Cu}(1)$ & $P(1)$ & $C(1)$ & $C(2)$ & $-162.7(3)$ & $\mathrm{Cu}(1)$ & $P(1)$ & $C(1)$ & $C(6)$ & $17.1(3)^{\prime}$ \\
\hline$C(7)$ & $P(1)$ & $C(1)$ & $C(2)$ & $75.4(3)$ & $C(7)$ & $P(1)$ & $C(1)$ & $C(6)$ & $-104.8(3)$ \\
\hline$C(13)$ & $P(1)$ & $C(1)$ & $C(2)$ & $-33.5(3)$ & $C(13)$ & $P(1)$ & $C(1)$ & $C(6)$ & $146.4(3)$ \\
\hline $\mathrm{Cu}(1)$ & $P(1)$ & $C(7)$ & $C(8)$ & $-145.6(3)$ & $\mathrm{Cu}(1)$ & $P(1)$ & $C(7)$ & $C(12)$ & $34.6(4)$ \\
\hline$C(1)$ & $P(1)$ & $C(7)$ & $C(8)$ & $-33.4(4)$ & $C(1)$ & $P(1)$ & $C(7)$ & $C(12)$ & $146.7(3)$ \\
\hline$C(13)$ & $P(1)$ & $C(7)$ & $C(8)$ & $75.7(4)$ & $C(13)$ & $P(1)$ & $C(7)$ & $C(12)$ & $-104.1(3)$ \\
\hline $\mathrm{Cu}(1)$ & $P(1)$ & $C(13)$ & $C(14)$ & $16.0(4)$ & $\mathrm{Cu}(1)$ & $P(1)$ & $C(13)$ & $C(18)$ & $-161.9(4)$ \\
\hline$C(1)$ & $P(1)$ & $C(13)$ & $C(14)$ & $-99.2(3)$ & $C(1)$ & $P(1)$ & $C(13)$ & $C(18)$ & $82.9(4)$ \\
\hline$C(7)$ & $P(1)$ & $C(13)$ & $C(14)$ & $151.0(3)$ & $C(7)$ & $P(1)$ & $C(13)$ & $C(18)$ & $-26.9(4)$ \\
\hline $\mathrm{Cu}(1)$ & $P(2)$ & $C(6)$ & $C(1)$ & $-18.3(3)$ & $\mathrm{Cu}(1)$ & $P(2)$ & $C(6)$ & $C(5)$ & $162.7(3)$ \\
\hline$C(19)$ & $P(2)$ & $C(6)$ & $C(1)$ & 106.3(3) & $C(19)$ & $P(2)$ & $C(6)$ & $C(5)$ & $-72.7(3)$ \\
\hline C(25) & $P(2)$ & $C(6)$ & $C(1)$ & $-145.0(3)$ & $C(25)$ & $P(2)$ & $C(6)$ & $C(5)$ & $36.0(3)$ \\
\hline $\mathrm{Cu}(1)$ & $P(2)$ & $C(19)$ & $C(20)$ & $-14.2(4)$ & $\mathrm{Cu}(1)$ & $P(2)$ & $C(19)$ & $C(24)$ & 168.6(3) \\
\hline
\end{tabular}

Table 8. Torsion angles $\left(^{\circ}\right)$-- continued

$\begin{array}{llllllllll}\text { atom1 } & \text { atom2 } & \text { atom3 } & \text { atom4 } & \text { angle } & \text { atom1 } & \text { atom2 } & \text { atom3 } & \text { atom4 } & \text { angle } \\ \mathrm{C}(6) & \mathrm{P}(2) & \mathrm{C}(19) & \mathrm{C}(20) & -126.5(3) & \mathrm{C}(6) & \mathrm{P}(2) & \mathrm{C}(19) & \mathrm{C}(24) & 56.2(4) \\ \mathrm{C}(25) & \mathrm{P}(2) & \mathrm{C}(19) & \mathrm{C}(20) & 125.5(3) & \mathrm{C}(25) & \mathrm{P}(2) & \mathrm{C}(19) & \mathrm{C}(24) & -51.7(4) \\ \mathrm{Cu}(1) & \mathrm{P}(2) & \mathrm{C}(25) & \mathrm{C}(26) & 121.8(3) & \mathrm{Cu}(1) & \mathrm{P}(2) & \mathrm{C}(25) & \mathrm{C}(30) & -52.9(4) \\ \mathrm{C}(6) & \mathrm{P}(2) & \mathrm{C}(25) & \mathrm{C}(26) & -124.5(4) & \mathrm{C}(6) & \mathrm{P}(2) & \mathrm{C}(25) & \mathrm{C}(30) & 60.8(4) \\ \mathrm{C}(19) & \mathrm{P}(2) & \mathrm{C}(25) & \mathrm{C}(26) & -17.1(4) & \mathrm{C}(19) & \mathrm{P}(2) & \mathrm{C}(25) & \mathrm{C}(30) & 168.2(3) \\ \mathrm{Cu}(2) & \mathrm{P}(3) & \mathrm{C}(31) & \mathrm{C}(32) & -152.8(3) & \mathrm{Cu}(2) & \mathrm{P}(3) & \mathrm{C}(31) & \mathrm{C}(36) & 29.7(3) \\ \mathrm{C}(37) & \mathrm{P}(3) & \mathrm{C}(31) & \mathrm{C}(32) & -21.3(3) & \mathrm{C}(37) & \mathrm{P}(3) & \mathrm{C}(31) & \mathrm{C}(36) & 161.3(3) \\ \mathrm{C}(43) & \mathrm{P}(3) & \mathrm{C}(31) & \mathrm{C}(32) & 88.8(3) & \mathrm{C}(43) & \mathrm{P}(3) & \mathrm{C}(31) & \mathrm{C}(36) & -88.6(3) \\ \mathrm{Cu}(2) & \mathrm{P}(3) & \mathrm{C}(37) & \mathrm{C}(38) & -121.2(4) & \mathrm{Cu}(2) & \mathrm{P}(3) & \mathrm{C}(37) & \mathrm{C}(42) & 50.6(5) \\ \mathrm{C}(31) & \mathrm{P}(3) & \mathrm{C}(37) & \mathrm{C}(38) & 123.6(4) & \mathrm{C}(31) & \mathrm{P}(3) & \mathrm{C}(37) & \mathrm{C}(42) & -64.7(4)\end{array}$




$\begin{array}{lllll}\mathrm{C}(43) & \mathrm{P}(3) & \mathrm{C}(37) & \mathrm{C}(38) & 17.0(4) \\ \mathrm{C}(2) & \mathrm{P}(3) & \mathrm{C}(43) & \mathrm{C}(44) & 18.7(4) \\ \mathrm{C}(31) & \mathrm{P}(3) & \mathrm{C}(43) & \mathrm{C}(44) & 126.5(3) \\ \mathrm{C}(37) & \mathrm{P}(3) & \mathrm{C}(43) & \mathrm{C}(44) & -124.7(3) \\ \mathrm{Cu}(2) & \mathrm{P}(4) & \mathrm{C}(36) & \mathrm{C}(31) & -21.1(3) \\ \mathrm{C}(49) & \mathrm{P}(4) & \mathrm{C}(36) & \mathrm{C}(31) & -151.9(3) \\ \mathrm{C}(55) & \mathrm{P}(4) & \mathrm{C}(36) & \mathrm{C}(31) & 97.5(3) \\ \mathrm{C}(2) & \mathrm{P}(4) & \mathrm{C}(49) & \mathrm{C}(50) & 148.7(4) \\ \mathrm{C}(36) & \mathrm{P}(4) & \mathrm{C}(49) & \mathrm{C}(50) & -95.6(4) \\ \mathrm{C}(55) & \mathrm{P}(4) & \mathrm{C}(49) & \mathrm{C}(50) & 14.2(5) \\ \mathrm{Cu}(2) & \mathrm{P}(4) & \mathrm{C}(55) & \mathrm{C}(56) & 120.1(3) \\ \mathrm{C}(36) & \mathrm{P}(4) & \mathrm{C}(55) & \mathrm{C}(56) & 10.2(4) \\ \mathrm{C}(49) & \mathrm{P}(4) & \mathrm{C}(55) & \mathrm{C}(56) & -99.8(3) \\ \mathrm{P}(1) & \mathrm{C}(1) & \mathrm{C}(2) & \mathrm{C}(3) & -179.3(3) \\ \mathrm{P}(1) & \mathrm{C}(1) & \mathrm{C}(6) & \mathrm{P}(2) & 0.8(4) \\ \mathrm{C}(2) & \mathrm{C}(1) & \mathrm{C}(6) & \mathrm{P}(2) & -179.4(3) \\ \mathrm{C}(1) & \mathrm{C}(2) & \mathrm{C}(3) & \mathrm{C}(4) & -0.6(6) \\ \mathrm{C}(3) & \mathrm{C}(4) & \mathrm{C}(5) & \mathrm{C}(6) & 0.6(6) \\ \mathrm{C}(4) & \mathrm{C}(5) & \mathrm{C}(6) & \mathrm{C}(1) & -0.4(6) \\ \mathrm{C}(12) & \mathrm{C}(7) & \mathrm{C}(8) & \mathrm{C}(9) & 1.2(6) \\ \mathrm{C}(8) & \mathrm{C}(7) & \mathrm{C}(12) & \mathrm{C}(11) & -0.5(6) \\ \mathrm{C}(8) & \mathrm{C}(9) & \mathrm{C}(10) & \mathrm{C}(11) & 0.6(7) \\ \mathrm{C}(10) & \mathrm{C}(11) & \mathrm{C}(12) & \mathrm{C}(7) & -0.2(5) \\ \mathrm{C}(18) & \mathrm{C}(13) & \mathrm{C}(14) & \mathrm{C}(15) & -1.1(7) \\ \mathrm{C}(14) & \mathrm{C}(13) & \mathrm{C}(18) & \mathrm{C}(17) & 2.3(7) \\ \mathrm{C}(14) & \mathrm{C}(15) & \mathrm{C}(16) & \mathrm{C}(17) & 0.1(6) \\ \mathrm{C}(16) & \mathrm{C}(17) & \mathrm{C}(18) & \mathrm{C}(13) & -2.4(8) \\ & & & & \end{array}$

$\begin{array}{lllll}\mathrm{C}(43) & \mathrm{P}(3) & \mathrm{C}(37) & \mathrm{C}(42) & -171.2(4) \\ \mathrm{Cu}(2) & \mathrm{P}(3) & \mathrm{C}(43) & \mathrm{C}(48) & -153.7(3) \\ \mathrm{C}(31) & \mathrm{P}(3) & \mathrm{C}(43) & \mathrm{C}(48) & -45.9(3) \\ \mathrm{C}(37) & \mathrm{P}(3) & \mathrm{C}(43) & \mathrm{C}(48) & 62.9(4) \\ \mathrm{Cu}(2) & \mathrm{P}(4) & \mathrm{C}(36) & \mathrm{C}(35) & 157.8(3) \\ \mathrm{C}(49) & \mathrm{P}(4) & \mathrm{C}(36) & \mathrm{C}(35) & 27.0(3) \\ \mathrm{C}(55) & \mathrm{P}(4) & \mathrm{C}(36) & \mathrm{C}(35) & -83.6(3) \\ \mathrm{Cu}(2) & \mathrm{P}(4) & \mathrm{C}(49) & \mathrm{C}(54) & -27.5(5) \\ \mathrm{C}(36) & \mathrm{P}(4) & \mathrm{C}(49) & \mathrm{C}(54) & 88.2(4) \\ \mathrm{C}(55) & \mathrm{P}(4) & \mathrm{C}(49) & \mathrm{C}(54) & -162.0(3) \\ \mathrm{Cu}(2) & \mathrm{P}(4) & \mathrm{C}(55) & \mathrm{C}(60) & -55.1(4) \\ \mathrm{C}(36) & \mathrm{P}(4) & \mathrm{C}(55) & \mathrm{C}(60) & -165.0(3) \\ \mathrm{C}(49) & \mathrm{P}(4) & \mathrm{C}(55) & \mathrm{C}(60) & 85.1(3) \\ \mathrm{C}(6) & \mathrm{C}(1) & \mathrm{C}(2) & \mathrm{C}(3) & 0.8(6) \\ \mathrm{P}(1) & \mathrm{C}(1) & \mathrm{C}(6) & \mathrm{C}(5) & 179.8(3) \\ \mathrm{C}(2) & \mathrm{C}(1) & \mathrm{C}(6) & \mathrm{C}(5) & -0.3(5) \\ \mathrm{C}(2) & \mathrm{C}(3) & \mathrm{C}(4) & \mathrm{C}(5) & -0.1(5) \\ \mathrm{C}(4) & \mathrm{C}(5) & \mathrm{C}(6) & \mathrm{P}(2) & 178.6(3) \\ \mathrm{P}(1) & \mathrm{C}(7) & \mathrm{C}(8) & \mathrm{C}(9) & -178.6(3) \\ \mathrm{P}(1) & \mathrm{C}(7) & \mathrm{C}(12) & \mathrm{C}(11) & 179.4(4) \\ \mathrm{C}(7) & \mathrm{C}(8) & \mathrm{C}(9) & \mathrm{C}(10) & -1.3(6) \\ \mathrm{C}(9) & \mathrm{C}(10) & \mathrm{C}(11) & \mathrm{C}(12) & 0.1(5) \\ \mathrm{P}(1) & \mathrm{C}(13) & \mathrm{C}(14) & \mathrm{C}(15) & -179.1(4) \\ \mathrm{P}(1) & \mathrm{C}(13) & \mathrm{C}(18) & \mathrm{C}(17) & -179.8(4) \\ \mathrm{C}(13) & \mathrm{C}(14) & \mathrm{C}(15) & \mathrm{C}(16) & -0.1(6) \\ \mathrm{C}(15) & \mathrm{C}(16) & \mathrm{C}(17) & \mathrm{C}(18) & 1.1(8) \\ \mathrm{P}(2) & \mathrm{C}(19) & \mathrm{C}(20) & \mathrm{C}(21) & -177.3(4) \\ & & & & \end{array}$

Table 8. Torsion angles $\left({ }^{0}\right)$-- continued

$\begin{array}{llllllllll}\text { atom1 } & \text { atom2 } & \text { atom3 } & \text { atom4 } & \text { angle } & \text { atom1 } & \text { atom2 } & \text { atom3 } & \text { atom4 } & \text { angle } \\ \mathrm{C}(24) & \mathrm{C}(19) & \mathrm{C}(20) & \mathrm{C}(21) & -0.0(6) & \mathrm{P}(2) & \mathrm{C}(19) & \mathrm{C}(24) & \mathrm{C}(23) & 177.3(4) \\ \mathrm{C}(20) & \mathrm{C}(19) & \mathrm{C}(24) & \mathrm{C}(23) & 0.0(5) & \mathrm{C}(19) & \mathrm{C}(20) & \mathrm{C}(21) & \mathrm{C}(22) & 0.6(7) \\ \mathrm{C}(20) & \mathrm{C}(21) & \mathrm{C}(22) & \mathrm{C}(23) & -1.3(7) & \mathrm{C}(21) & \mathrm{C}(22) & \mathrm{C}(23) & \mathrm{C}(24) & 1.3(7) \\ \mathrm{C}(22) & \mathrm{C}(23) & \mathrm{C}(24) & \mathrm{C}(19) & -0.7(7) & \mathrm{P}(2) & \mathrm{C}(25) & \mathrm{C}(26) & \mathrm{C}(27) & -176.2(4) \\ \mathrm{C}(30) & \mathrm{C}(25) & \mathrm{C}(26) & \mathrm{C}(27) & -1.5(7) & \mathrm{P}(2) & \mathrm{C}(25) & \mathrm{C}(30) & \mathrm{C}(29) & 176.5(4) \\ \mathrm{C}(26) & \mathrm{C}(25) & \mathrm{C}(30) & \mathrm{C}(29) & 1.5(7) & \mathrm{C}(25) & \mathrm{C}(26) & \mathrm{C}(27) & \mathrm{C}(28) & -1.1(7) \\ \mathrm{C}(26) & \mathrm{C}(27) & \mathrm{C}(28) & \mathrm{C}(29) & 3.7(8) & \mathrm{C}(27) & \mathrm{C}(28) & \mathrm{C}(29) & \mathrm{C}(30) & -3.8(8) \\ \mathrm{C}(28) & \mathrm{C}(29) & \mathrm{C}(30) & \mathrm{C}(25) & 1.1(8) & \mathrm{P}(3) & \mathrm{C}(31) & \mathrm{C}(32) & \mathrm{C}(33) & -175.5(3) \\ \mathrm{C}(36) & \mathrm{C}(31) & \mathrm{C}(32) & \mathrm{C}(33) & 1.8(5) & \mathrm{P}(3) & \mathrm{C}(31) & \mathrm{C}(36) & \mathrm{P}(4) & -5.9(4) \\ \mathrm{P}(3) & \mathrm{C}(31) & \mathrm{C}(36) & \mathrm{C}(35) & 175.1(3) & \mathrm{C}(32) & \mathrm{C}(31) & \mathrm{C}(36) & \mathrm{P}(4) & 176.5(3) \\ \mathrm{C}(32) & \mathrm{C}(31) & \mathrm{C}(36) & \mathrm{C}(35) & -2.4(5) & \mathrm{C}(31) & \mathrm{C}(32) & \mathrm{C}(33) & \mathrm{C}(34) & 0.4(6) \\ \mathrm{C}(32) & \mathrm{C}(33) & \mathrm{C}(34) & \mathrm{C}(35) & -2.0(6) & \mathrm{C}(33) & \mathrm{C}(34) & \mathrm{C}(35) & \mathrm{C}(36) & 1.3(6) \\ \mathrm{C}(34) & \mathrm{C}(35) & \mathrm{C}(36) & \mathrm{P}(4) & -178.1(3) & \mathrm{C}(34) & \mathrm{C}(35) & \mathrm{C}(36) & \mathrm{C}(31) & 0.8(5) \\ \mathrm{P}(3) & \mathrm{C}(37) & \mathrm{C}(38) & \mathrm{C}(39) & 170.7(4) & \mathrm{C}(42) & \mathrm{C}(37) & \mathrm{C}(38) & \mathrm{C}(39) & -1.1(7) \\ \mathrm{P}(3) & \mathrm{C}(37) & \mathrm{C}(42) & \mathrm{C}(41) & -171.8(4) & \mathrm{C}(38) & \mathrm{C}(37) & \mathrm{C}(42) & \mathrm{C}(41) & 0.3(7) \\ \mathrm{C}(37) & \mathrm{C}(38) & \mathrm{C}(39) & \mathrm{C}(40) & 1.5(8) & \mathrm{C}(38) & \mathrm{C}(39) & \mathrm{C}(40) & \mathrm{C}(41) & -1.1(9) \\ \mathrm{C}(39) & \mathrm{C}(40) & \mathrm{C}(41) & \mathrm{C}(42) & 0.3(7) & \mathrm{C}(40) & \mathrm{C}(41) & \mathrm{C}(42) & \mathrm{C}(37) & 0.1(7) \\ \mathrm{P}(3) & \mathrm{C}(43) & \mathrm{C}(44) & \mathrm{C}(45) & -172.4(3) & \mathrm{C}(48) & \mathrm{C}(43) & \mathrm{C}(44) & \mathrm{C}(45) & 0.0(5) \\ \mathrm{P}(3) & \mathrm{C}(43) & \mathrm{C}(48) & \mathrm{C}(47) & 172.9(3) & \mathrm{C}(44) & \mathrm{C}(43) & \mathrm{C}(48) & \mathrm{C}(47) & 0.6(6) \\ \mathrm{C}(43) & \mathrm{C}(44) & \mathrm{C}(45) & \mathrm{C}(46) & -0.0(6) & \mathrm{C}(44) & \mathrm{C}(45) & \mathrm{C}(46) & \mathrm{C}(47) & -0.7(7)\end{array}$




$\begin{array}{llllllllll}\mathrm{C}(45) & \mathrm{C}(46) & \mathrm{C}(47) & \mathrm{C}(48) & 1.4(7) & \mathrm{C}(46) & \mathrm{C}(47) & \mathrm{C}(48) & \mathrm{C}(43) & -1.3(6) \\ \mathrm{P}(4) & \mathrm{C}(49) & \mathrm{C}(50) & \mathrm{C}(51) & -174.5(4) & \mathrm{C}(54) & \mathrm{C}(49) & \mathrm{C}(50) & \mathrm{C}(51) & 1.7(7) \\ \mathrm{P}(4) & \mathrm{C}(49) & \mathrm{C}(54) & \mathrm{C}(53) & 176.4(4) & \mathrm{C}(50) & \mathrm{C}(49) & \mathrm{C}(54) & \mathrm{C}(53) & -0.1(6) \\ \mathrm{C}(49) & \mathrm{C}(50) & \mathrm{C}(51) & \mathrm{C}(52) & -2.0(8) & \mathrm{C}(50) & \mathrm{C}(51) & \mathrm{C}(52) & \mathrm{C}(53) & 0.7(8) \\ \mathrm{C}(51) & \mathrm{C}(52) & \mathrm{C}(53) & \mathrm{C}(54) & 0.8(8) & \mathrm{C}(52) & \mathrm{C}(53) & \mathrm{C}(54) & \mathrm{C}(49) & -1.2(8) \\ \mathrm{P}(4) & \mathrm{C}(55) & \mathrm{C}(56) & \mathrm{C}(57) & -175.2(4) & \mathrm{C}(60) & \mathrm{C}(55) & \mathrm{C}(56) & \mathrm{C}(57) & -0.1(5) \\ \mathrm{P}(4) & \mathrm{C}(55) & \mathrm{C}(60) & \mathrm{C}(59) & 175.2(4) & \mathrm{C}(56) & \mathrm{C}(55) & \mathrm{C}(60) & \mathrm{C}(59) & -0.1(5) \\ \mathrm{C}(55) & \mathrm{C}(56) & \mathrm{C}(57) & \mathrm{C}(58) & 0.9(7) & \mathrm{C}(56) & \mathrm{C}(57) & \mathrm{C}(58) & \mathrm{C}(59) & -1.5(7) \\ \mathrm{C}(57) & \mathrm{C}(58) & \mathrm{C}(59) & \mathrm{C}(60) & 1.2(7) & \mathrm{C}(58) & \mathrm{C}(59) & \mathrm{C}(60) & \mathrm{C}(55) & -0.4(6)\end{array}$

The sign is positive if when looking from atom 2 to atom 3 a clock-wise motion of atom 1 would superimpose it on atom 4. 


\section{$[\mathrm{Cu}(\mu \mathrm{Cl}) \mathrm{dppb}]_{2}(\mathbf{3})$}

\section{Table S5.}

\section{EXPERIMENTAL DETAILS}

A. Crystal Data

\author{
Empirical Formula \\ Formula Weight \\ Crystal Color, Habit \\ Crystal Dimensions \\ Crystal System \\ Lattice Type \\ Indexing Images \\ Detector Position \\ Pixel Size
}

Lattice Parameters

Space Group

$Z$ value

$D_{\text {calc }}$

$\mathrm{F}_{000}$

$\mu(\operatorname{MoK} \alpha)$

Diffractometer

Radiation

Detector Aperture

Data Images

$\omega$ oscillation Range $(\chi=45.0, \phi=30.0)$

Exposure Rate

$\omega$ oscillation Range ( $\chi=45.0, \phi=180.0)$

Exposure Rate

Detector Position

Pixel Size

$2 \theta \max$

\author{
$\mathrm{C}_{60} \mathrm{H}_{48} \mathrm{P}_{4} \mathrm{Cu}_{2} \mathrm{Cl}_{2}$ \\ 1090.93 \\ yellow, platelet \\ $0.30 \times 0.25 \times 0.10 \mathrm{~mm}$ \\ monoclinic \\ Primitive \\ 3 oscillations @ 90.0 seconds \\ $127.40 \mathrm{~mm}$ \\ $0.100 \mathrm{~mm}$
}

$$
\begin{aligned}
& a=11.137(8) \AA \\
& b=18.74(2) \AA \\
& c=12.303(8) \AA \\
& \beta=97.45(3) \AA \\
& V=2545(3) \AA^{3}
\end{aligned}
$$

$\mathrm{P} 21(\# 4)$

2

$1.423 \mathrm{~g} / \mathrm{cm}^{3}$

1120.00

$11.06 \mathrm{~cm}^{-1}$

B. Intensity Measurements

Rigaku RAXIS-RAPID

$\operatorname{MoK} \alpha(\lambda=0.71075 \AA)$

graphite monochromated

$280 \mathrm{~mm} \times 256 \mathrm{~mm}$

83 exposures

$130.0-190.0^{\circ}$

$30.0 \mathrm{sec} . / \mathrm{O}$

$0.0-159.0^{\circ}$

$30.0 \mathrm{sec} . / \mathrm{O}$

$127.40 \mathrm{~mm}$

$0.100 \mathrm{~mm}$

54.90 
No. of Reflections Measured

Corrections
Total: 23769

Unique: $11026\left(R_{\text {int }}=0.058\right)$

Lorentz-polarization

Absorption

(trans. factors: $0.7248-1.0000$ )

C. Structure Solution and Refinement
Structure Solution

Refinement

Function Minimized

Least Squares Weights

$2 \theta$ max cutoff

Anomalous Dispersion

No. Observations (All reflections)

No. Variables

Reflection/Parameter Ratio

Residuals: $\mathrm{R}$ (All reflections)

Residuals: R1 $(\mathrm{I}>2.00 \sigma(\mathrm{I}))$

Residuals: wR2 (All reflections)

Goodness of Fit Indicator

Max Shift/Error in Final Cycle

Maximum peak in Final Diff. Map

Minimum peak in Final Diff. Map
Patterson Methods (DIRDIF99 PATTY)

Full-matrix least-squares on $\mathrm{F}^{2}$

$\Sigma \mathrm{w}\left(\mathrm{Fo}^{2}-\mathrm{Fc}^{2}\right)^{2}$

$1 /\left[0.7720 \sigma\left(\mathrm{Fo}^{2}\right)\right] /\left(4 \mathrm{Fo}^{2}\right)$

$54.9^{\circ}$

All non-hydrogen atoms

11026

661

16.68

0.088

0.042

0.052

0.960

0.000

$1.51 \mathrm{e}^{-/} / \mathrm{A}^{3}$

$-1.01 e^{-} / \AA^{3}$

Table 4. Bond lengths $(\AA)$

$\begin{array}{llllll}\text { atom } & \text { atom } & \text { distance } & \text { atom } & \text { atom } & \text { distance } \\ \mathrm{Cu}(1) & \mathrm{Cl}(1) & 2.383(1) & \mathrm{Cu}(1) & \mathrm{Cl}(2) & 2.316(1) \\ \mathrm{Cu}(1) & \mathrm{P}(1) & 2.255(2) & \mathrm{Cu}(1) & \mathrm{P}(2) & 2.260(2) \\ \mathrm{Cu}(2) & \mathrm{Cl}(1) & 2.324(1) & \mathrm{Cu}(2) & \mathrm{Cl}(2) & 2.414(1) \\ \mathrm{Cu}(2) & \mathrm{P}(3) & 2.246(2) & \mathrm{Cu}(2) & \mathrm{P}(4) & 2.255(1) \\ \mathrm{P}(1) & \mathrm{C}(1) & 1.829(5) & \mathrm{P}(1) & \mathrm{C}(7) & 1.814(5) \\ \mathrm{P}(1) & \mathrm{C}(25) & 1.837(4) & \mathrm{P}(2) & \mathrm{C}(13) & 1.817(5) \\ \mathrm{P}(2) & \mathrm{C}(19) & 1.802(6) & \mathrm{P}(2) & \mathrm{C}(30) & 1.831(5) \\ \mathrm{P}(3) & \mathrm{C}(31) & 1.825(5) & \mathrm{P}(3) & \mathrm{C}(37) & 1.804(5) \\ \mathrm{P}(3) & \mathrm{C}(60) & 1.848(4) & \mathrm{P}(4) & \mathrm{C}(43) & 1.824(4) \\ \mathrm{P}(4) & \mathrm{C}(49) & 1.809(5) & \mathrm{P}(4) & \mathrm{C}(55) & 1.845(5) \\ \mathrm{C}(1) & \mathrm{C}(2) & 1.390(7) & \mathrm{C}(1) & \mathrm{C}(6) & 1.369(6) \\ \mathrm{C}(2) & \mathrm{C}(3) & 1.383(9) & \mathrm{C}(3) & \mathrm{C}(4) & 1.381(8) \\ \mathrm{C}(4) & \mathrm{C}(5) & 1.357(9) & \mathrm{C}(5) & \mathrm{C}(6) & 1.383(8) \\ \mathrm{C}(7) & \mathrm{C}(8) & 1.377(8) & \mathrm{C}(7) & \mathrm{C}(12) & 1.362(8) \\ \mathrm{C}(8) & \mathrm{C}(9) & 1.358(9) & \mathrm{C}(9) & \mathrm{C}(10) & 1.376(9) \\ \mathrm{C}(10) & \mathrm{C}(9) & 1.376(9) & \mathrm{C}(10) & \mathrm{C}(11) & 1.36(1) \\ \mathrm{C}(11) & \mathrm{C}(10) & 1.36(1) & \mathrm{C}(11) & \mathrm{C}(12) & 1.374(9) \\ \mathrm{C}(13) & \mathrm{C}(14) & 1.370(8) & \mathrm{C}(13) & \mathrm{C}(18) & 1.385(7) \\ \mathrm{C}(14) & \mathrm{C}(15) & 1.382(9) & \mathrm{C}(15) & \mathrm{C}(16) & 1.363(9)\end{array}$




\begin{tabular}{|c|c|c|c|c|c|c|c|}
\hline$C(16)$ & \multicolumn{2}{|l|}{$\mathrm{C}(17)$} & $1.37(1)$ & \multicolumn{2}{|c|}{$C(17)$} & $C(18)$ & $1.384(8)$ \\
\hline$C(19)$ & \multicolumn{2}{|l|}{$C(20)$} & $1.361(9)$ & \multicolumn{2}{|c|}{$C(19)$} & $C(24)$ & $1.383(9)$ \\
\hline$C(20)$ & \multicolumn{2}{|l|}{$C(21)$} & $1.39(1)$ & \multicolumn{2}{|c|}{$C(21)$} & $C(22)$ & $1.34(1)$ \\
\hline$C(22)$ & \multicolumn{2}{|l|}{$C(23)$} & $1.36(1)$ & \multicolumn{2}{|c|}{$C(23)$} & $C(24)$ & $1.362(9)$ \\
\hline$C(25)$ & \multicolumn{2}{|l|}{$C(26)$} & $1.362(7)$ & \multicolumn{2}{|c|}{$C(25)$} & $C(30)$ & $1.396(7)$ \\
\hline$C(26)$ & \multicolumn{2}{|l|}{$C(27)$} & $1.373(7)$ & \multicolumn{2}{|c|}{$C(27)$} & $C(28)$ & $1.36(1)$ \\
\hline$C(28)$ & \multicolumn{2}{|l|}{$C(29)$} & $1.385(7)$ & $\mathrm{C}(2$ & & $C(30)$ & $1.401(7)$ \\
\hline$C(31)$ & $C(32)$ & & $1.381(7)$ & $C(3$ & & $C(36)$ & $1.382(8)$ \\
\hline$C(32)$ & $C(33)$ & & $1.377(8)$ & $C(3$ & & $C(34)$ & $1.38(1)$ \\
\hline$C(34)$ & $C(35)$ & & $1.35(1)$ & $C(3$ & & $C(34)$ & $1.35(1)$ \\
\hline$C(35)$ & $C(36)$ & & $1.357(9)$ & $C(3$ & & $C(35)$ & $1.357(9)$ \\
\hline$C(37)$ & $C(38)$ & & $1.373(7)$ & $C(3$ & & $C(42)$ & $1.383(7)$ \\
\hline$C(38)$ & $C(39)$ & & $1.379(8)$ & $C(3$ & & $C(40)$ & $1.352(9)$ \\
\hline$C(40)$ & $C(41)$ & & 1.352(9) & $C(4$ & & $C(42)$ & $1.399(9)$ \\
\hline$C(43)$ & $C(44)$ & & $1.365(7)$ & $\mathrm{C}(4$ & & $C(48)$ & $1.374(6)$ \\
\hline C(44) & $C(45)$ & & $1.391(7)$ & $\mathrm{C}(4$ & & $C(46)$ & $1.340(7)$ \\
\hline$C(46)$ & $C(47)$ & & $1.371(8)$ & $\mathrm{C}(4$ & & $C(48)$ & $1.382(7)$ \\
\hline $\mathrm{C}(49)$ & $C(50)$ & & $1.384(7)$ & $C(4$ & & C(54) & $1.383(7)$ \\
\hline Table 4 & 3ond leng & $(\AA)$ & -- continued & & & & \\
\hline atom & atom & & distance & ato & & atom & distance \\
\hline$C(50)$ & $C(51)$ & & $1.366(8)$ & $C(5$ & & $C(52)$ & $1.358(8)$ \\
\hline$C(52)$ & $C(53)$ & & $1.353(9)$ & $C(5$ & & $C(54)$ & $1.399(9)$ \\
\hline$C(55)$ & $C(56)$ & & $1.384(6)$ & $C(5$ & & $C(60)$ & $1.403(6)$ \\
\hline$C(56)$ & $C(57)$ & & $1.362(7)$ & $C(5$ & & C(58) & $1.387(8)$ \\
\hline$C(58)$ & $C(59)$ & & $1.378(6)$ & $C(5$ & & $C(60)$ & $1.396(7)$ \\
\hline Table 6 & 3ond ang & $\left.{ }^{(}\right)^{\circ}$ & & & & & \\
\hline atom & atom & atom & angle & atom & atom & atom & angle \\
\hline $\mathrm{Cu}(1)$ & $\mathrm{Cl}(1)$ & $\mathrm{Cu}(2)$ & 74.99(4) & $\mathrm{Cl}(2)$ & $\mathrm{Cu}(1)$ & $\mathrm{Cl}(1)$ & $102.87(4)$ \\
\hline$P(1)$ & $\mathrm{Cu}(1)$ & $\mathrm{Cl}(1)$ & $112.02(6)$ & $P(2)$ & $\mathrm{Cu}(1)$ & $\mathrm{Cl}(1)$ & $112.21(5)$ \\
\hline $\mathrm{Cu}(1)$ & $\mathrm{Cl}(2)$ & $\mathrm{Cu}(2)$ & $74.54(4)$ & $P(1)$ & $\mathrm{Cu}(1)$ & $\mathrm{Cl}(2)$ & $118.75(6)$ \\
\hline$P(2)$ & $\mathrm{Cu}(1)$ & $\mathrm{Cl}(2)$ & $123.49(6)$ & $P(2)$ & $\mathrm{Cu}(1)$ & $P(1)$ & $87.50(5)$ \\
\hline $\mathrm{Cu}(1)$ & $P(1)$ & $C(1)$ & $114.4(2)$ & $\mathrm{Cu}(1)$ & $P(1)$ & $C(25)$ & 101.8(2) \\
\hline $\mathrm{Cu}(1)$ & $P(1)$ & $C(7)$ & $124.8(2)$ & $\mathrm{Cu}(1)$ & $\mathrm{P}(2)$ & $C(13)$ & $114.0(2)$ \\
\hline $\mathrm{Cu}(1)$ & $P(2)$ & $C(30)$ & $100.6(2)$ & $\mathrm{Cu}(1)$ & $P(2)$ & $C(19)$ & $126.1(2)$ \\
\hline $\mathrm{Cl}(2)$ & $\mathrm{Cu}(2)$ & $\mathrm{Cl}(1)$ & $101.67(4)$ & $P(3)$ & $\mathrm{Cu}(2)$ & $\mathrm{Cl}(1)$ & $122.47(6)$ \\
\hline$P(4)$ & $\mathrm{Cu}(2)$ & $\mathrm{Cl}(1)$ & $121.40(5)$ & $P(3)$ & $\mathrm{Cu}(2)$ & $\mathrm{Cl}(2)$ & 109.31(5) \\
\hline $\mathrm{P}(4)$ & $\mathrm{Cu}(2)$ & $\mathrm{Cl}(2)$ & $111.36(5)$ & $P(4)$ & $\mathrm{Cu}(2)$ & $P(3)$ & $90.56(5)$ \\
\hline $\mathrm{Cu}(2)$ & $P(3)$ & $C(31)$ & $121.3(2)$ & $\mathrm{Cu}(2)$ & $P(3)$ & $C(37)$ & 119.6(2) \\
\hline $\mathrm{Cu}(2)$ & $P(3)$ & $C(60)$ & $102.3(2)$ & $\mathrm{Cu}(2)$ & $P(4)$ & $C(43)$ & 115.8(1) \\
\hline $\mathrm{Cu}(2)$ & $P(4)$ & $C(49)$ & $122.5(2)$ & $\mathrm{Cu}(2)$ & $P(4)$ & C(55) & $102.9(1)$ \\
\hline$C(25)$ & $P(1)$ & $C(1)$ & $103.8(2)$ & $P(1)$ & $C(1)$ & $C(2)$ & 116.1(4) \\
\hline$P(1)$ & $C(1)$ & $C(6)$ & $124.7(4)$ & $C(7)$ & $P(1)$ & $C(1)$ & $105.1(2)$ \\
\hline$C(25)$ & $P(1)$ & $\mathrm{C}(7)$ & $104.5(2)$ & $P(1)$ & $C(7)$ & $\mathrm{C}(8)$ & $117.9(4)$ \\
\hline$P(1)$ & $C(7)$ & $C(12)$ & $124.2(4)$ & $P(1)$ & $C(25)$ & $C(30)$ & 117.7(3) \\
\hline$P(1)$ & $C(25)$ & $C(26)$ & $123.1(4)$ & $P(2)$ & $C(13)$ & $C(14)$ & $118.6(4)$ \\
\hline$C(30)$ & $P(2)$ & $C(13)$ & $100.7(2)$ & $P(2)$ & $C(13)$ & $C(18)$ & $122.8(4)$ \\
\hline
\end{tabular}




$\begin{array}{llllllll}\mathrm{C}(19) & \mathrm{P}(2) & \mathrm{C}(13) & 106.5(2) & \mathrm{C}(30) & \mathrm{P}(2) & \mathrm{C}(19) & 105.3(2) \\ \mathrm{P}(2) & \mathrm{C}(19) & \mathrm{C}(20) & 125.7(5) & \mathrm{P}(2) & \mathrm{C}(19) & \mathrm{C}(24) & 118.0(4) \\ \mathrm{P}(2) & \mathrm{C}(30) & \mathrm{C}(25) & 118.2(3) & \mathrm{P}(2) & \mathrm{C}(30) & \mathrm{C}(29) & 123.0(4) \\ \mathrm{C}(37) & \mathrm{P}(3) & \mathrm{C}(31) & 104.9(2) & \mathrm{C}(60) & \mathrm{P}(3) & \mathrm{C}(31) & 103.3(2) \\ \mathrm{P}(3) & \mathrm{C}(31) & \mathrm{C}(32) & 117.9(4) & \mathrm{P}(3) & \mathrm{C}(31) & \mathrm{C}(36) & 124.2(4) \\ \mathrm{C}(60) & \mathrm{P}(3) & \mathrm{C}(37) & 102.5(2) & \mathrm{P}(3) & \mathrm{C}(37) & \mathrm{C}(38) & 123.4(4) \\ \mathrm{P}(3) & \mathrm{C}(37) & \mathrm{C}(42) & 119.9(4) & \mathrm{P}(3) & \mathrm{C}(60) & \mathrm{C}(55) & 119.8(3) \\ \mathrm{P}(3) & \mathrm{C}(60) & \mathrm{C}(59) & 122.0(3) & \mathrm{C}(49) & \mathrm{P}(4) & \mathrm{C}(43) & 104.1(2) \\ \mathrm{C}(55) & \mathrm{P}(4) & \mathrm{C}(43) & 105.0(2) & \mathrm{P}(4) & \mathrm{C}(43) & \mathrm{C}(44) & 117.6(3) \\ \mathrm{P}(4) & \mathrm{C}(43) & \mathrm{C}(48) & 125.0(4) & \mathrm{C}(55) & \mathrm{P}(4) & \mathrm{C}(49) & 104.8(2) \\ \mathrm{P}(4) & \mathrm{C}(49) & \mathrm{C}(50) & 117.3(3) & \mathrm{P}(4) & \mathrm{C}(49) & \mathrm{C}(54) & 125.8(4) \\ \mathrm{P}(4) & \mathrm{C}(55) & \mathrm{C}(60) & 118.4(3) & \mathrm{P}(4) & \mathrm{C}(55) & \mathrm{C}(56) & 121.7(4) \\ \mathrm{C}(6) & \mathrm{C}(1) & \mathrm{C}(2) & 119.1(5) & \mathrm{C}(1) & \mathrm{C}(2) & \mathrm{C}(3) & 119.9(5) \\ \mathrm{C}(1) & \mathrm{C}(6) & \mathrm{C}(5) & 120.4(5) & \mathrm{C}(2) & \mathrm{C}(3) & \mathrm{C}(4) & 120.26) \\ \mathrm{C}(3) & \mathrm{C}(4) & \mathrm{C}(5) & 119.5(6) & \mathrm{C}(4) & \mathrm{C}(5) & \mathrm{C}(6) & 120.8(5) \\ \mathrm{C}(12) & \mathrm{C}(7) & \mathrm{C}(8) & 117.9(5) & \mathrm{C}(7) & \mathrm{C}(8) & \mathrm{C}(9) & 121.3(5) \\ \mathrm{C}(7) & \mathrm{C}(12) & \mathrm{C}(11) & 121.3(5) & \mathrm{C}(8) & \mathrm{C}(9) & \mathrm{C}(10) & 120.3(6) \\ \mathrm{C}(9) & \mathrm{C}(10) & \mathrm{C}(11) & 118.9(6) & \mathrm{C}(10) & \mathrm{C}(11) & \mathrm{C}(12) & 120.2(6)\end{array}$

Table 6. Bond angles $\left(^{\circ}\right)$-- continued

$\begin{array}{llllllll}\text { atom } & \text { atom } & \text { atom } & \text { angle } & \text { atom } & \text { atom } & \text { atom } & \text { angle } \\ \mathrm{C}(18) & \mathrm{C}(13) & \mathrm{C}(14) & 118.2(5) & \mathrm{C}(13) & \mathrm{C}(14) & \mathrm{C}(15) & 121.4(5) \\ \mathrm{C}(13) & \mathrm{C}(18) & \mathrm{C}(17) & 120.8(5) & \mathrm{C}(14) & \mathrm{C}(15) & \mathrm{C}(16) & 119.5(6) \\ \mathrm{C}(15) & \mathrm{C}(16) & \mathrm{C}(17) & 120.6(6) & \mathrm{C}(16) & \mathrm{C}(17) & \mathrm{C}(18) & 119.4(5) \\ \mathrm{C}(24) & \mathrm{C}(19) & \mathrm{C}(20) & 116.0(5) & \mathrm{C}(19) & \mathrm{C}(20) & \mathrm{C}(21) & 121.4(6) \\ \mathrm{C}(19) & \mathrm{C}(24) & \mathrm{C}(23) & 121.9(6) & \mathrm{C}(20) & \mathrm{C}(21) & \mathrm{C}(22) & 121.8(7) \\ \mathrm{C}(21) & \mathrm{C}(22) & \mathrm{C}(23) & 117.5(7) & \mathrm{C}(22) & \mathrm{C}(23) & \mathrm{C}(24) & 121.4(6) \\ \mathrm{C}(30) & \mathrm{C}(25) & \mathrm{C}(26) & 119.2(4) & \mathrm{C}(25) & \mathrm{C}(26) & \mathrm{C}(27) & 121.9(5) \\ \mathrm{C}(25) & \mathrm{C}(30) & \mathrm{C}(29) & 118.7(4) & \mathrm{C}(26) & \mathrm{C}(27) & \mathrm{C}(28) & 120.0(5) \\ \mathrm{C}(27) & \mathrm{C}(28) & \mathrm{C}(29) & 119.8(5) & \mathrm{C}(28) & \mathrm{C}(29) & \mathrm{C}(30) & 120.4(5) \\ \mathrm{C}(36) & \mathrm{C}(31) & \mathrm{C}(32) & 117.7(5) & \mathrm{C}(31) & \mathrm{C}(32) & \mathrm{C}(33) & 120.6(5) \\ \mathrm{C}(31) & \mathrm{C}(36) & \mathrm{C}(35) & 121.8(5) & \mathrm{C}(32) & \mathrm{C}(33) & \mathrm{C}(34) & 119.6(6) \\ \mathrm{C}(33) & \mathrm{C}(34) & \mathrm{C}(35) & 120.2(6) & \mathrm{C}(34) & \mathrm{C}(35) & \mathrm{C}(36) & 120.0(6) \\ \mathrm{C}(42) & \mathrm{C}(37) & \mathrm{C}(38) & 116.7(5) & \mathrm{C}(37) & \mathrm{C}(38) & \mathrm{C}(39) & 123.0(5) \\ \mathrm{C}(37) & \mathrm{C}(42) & \mathrm{C}(41) & 120.4(5) & \mathrm{C}(38) & \mathrm{C}(39) & \mathrm{C}(40) & 118.9(5) \\ \mathrm{C}(39) & \mathrm{C}(40) & \mathrm{C}(41) & 120.7(6) & \mathrm{C}(40) & \mathrm{C}(41) & \mathrm{C}(42) & 120.3(6) \\ \mathrm{C}(48) & \mathrm{C}(43) & \mathrm{C}(44) & 117.3(4) & \mathrm{C}(43) & \mathrm{C}(44) & \mathrm{C}(45) & 122.0(4) \\ \mathrm{C}(43) & \mathrm{C}(48) & \mathrm{C}(47) & 121.1(5) & \mathrm{C}(44) & \mathrm{C}(45) & \mathrm{C}(46) & 119.4(5) \\ \mathrm{C}(45) & \mathrm{C}(46) & \mathrm{C}(47) & 120.3(5) & \mathrm{C}(46) & \mathrm{C}(47) & \mathrm{C}(48) & 119.7(5) \\ \mathrm{C}(54) & \mathrm{C}(49) & \mathrm{C}(50) & 116.9(5) & \mathrm{C}(49) & \mathrm{C}(50) & \mathrm{C}(51) & 121.7(4) \\ \mathrm{C}(49) & \mathrm{C}(54) & \mathrm{C}(53) & 120.9(5) & \mathrm{C}(50) & \mathrm{C}(51) & \mathrm{C}(52) & 120.6(5) \\ \mathrm{C}(51) & \mathrm{C}(52) & \mathrm{C}(53) & 119.9(6) & \mathrm{C}(52) & \mathrm{C}(53) & \mathrm{C}(54) & 120.0(5) \\ \mathrm{C}(60) & \mathrm{C}(55) & \mathrm{C}(56) & 119.9(4) & \mathrm{C}(55) & \mathrm{C}(56) & \mathrm{C}(57) & 120.8(4) \\ \mathrm{C}(55) & \mathrm{C}(60) & \mathrm{C}(59) & 118.3(4) & \mathrm{C}(56) & \mathrm{C}(57) & \mathrm{C}(58) & 120.5(4) \\ \mathrm{C}(57) & \mathrm{C}(58) & \mathrm{C}(59) & 119.3(5) & \mathrm{C}(58) & \mathrm{C}(59) & \mathrm{C}(60) & 121.2(4)\end{array}$

Table 8. Torsion Angles $\left({ }^{\circ}\right)$

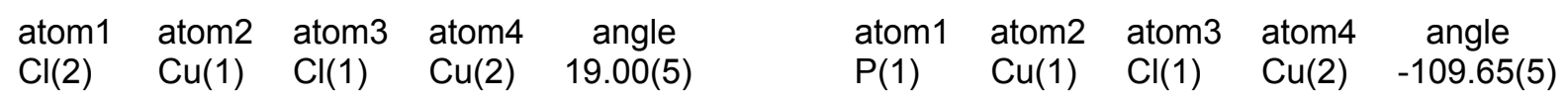




\begin{tabular}{|c|c|c|c|c|c|c|c|c|c|}
\hline$P(2)$ & $\mathrm{Cu}(1)$ & $\mathrm{Cl}(1)$ & $\mathrm{Cu}(2)$ & $153.80(5)$ & $\mathrm{Cl}(1)$ & $\mathrm{Cu}(1)$ & $\mathrm{Cl}(2)$ & $\mathrm{Cu}(2)$ & $-18.31(5)$ \\
\hline$P(1)$ & $\mathrm{Cu}(1)$ & $\mathrm{Cl}(2)$ & $\mathrm{Cu}(2)$ & 106.02(5) & $P(2)^{\prime}$ & $\mathrm{Cu}(1)$ & $\mathrm{Cl}(2)$ & $\mathrm{Cu}(2)$ & $-146.33(5)$ \\
\hline $\mathrm{Cl}(1)$ & $\mathrm{Cu}(1)$ & $P(1)$ & $C(1)$ & $166.0(2)$ & $\mathrm{Cl}(1)$ & $\mathrm{Cu}(1)$ & $P(1)$ & $C(7)$ & $34.4(2)$ \\
\hline $\mathrm{Cl}(1)$ & $\mathrm{Cu}(1)$ & $P(1)$ & $C(25)$ & $-82.7(1)$ & $\mathrm{Cl}(2)$ & $\mathrm{Cu}(1)$ & $P(1)$ & $C(1)$ & $46.3(2)$ \\
\hline $\mathrm{Cl}(2)$ & $\mathrm{Cu}(1)$ & $P(1)$ & $C(7)$ & $-85.3(2)$ & $\mathrm{Cl}(2)$ & $\mathrm{Cu}(1)$ & $P(1)$ & $C(25)$ & $157.5(1)$ \\
\hline$P(2)$ & $\mathrm{Cu}(1)$ & $P(1)$ & $\mathrm{C}(1)$ & $-81.0(2)$ & $P(2)^{\prime}$ & $\mathrm{Cu}(1)$ & $P(1)$ & $C(7)$ & 147.4(2) \\
\hline$P(2)$ & $\mathrm{Cu}(1)$ & $P(1)$ & $C(25)$ & $30.2(1)$ & $\mathrm{Cl}(1)$ & $\mathrm{Cu}(1)$ & $P(2)$ & $C(13)$ & $-172.8(2)$ \\
\hline $\mathrm{Cl}(1)$ & $\mathrm{Cu}(1)$ & $P(2)$ & $C(19)$ & $-37.6(2)$ & $\mathrm{Cl}(1)$ & $\mathrm{Cu}(1)$ & $P(2)$ & $C(30)$ & $80.3(2)$ \\
\hline $\mathrm{Cl}(2)$ & $\mathrm{Cu}(1)$ & $P(2)$ & $C(13)$ & $-48.8(2)$ & $\mathrm{Cl}(2)$ & $\mathrm{Cu}(1)$ & $P(2)$ & C(19) & $86.3(2)$ \\
\hline $\mathrm{Cl}(2)$ & $\mathrm{Cu}(1)$ & $P(2)$ & $C(30)$ & $-155.7(2)$ & $P(1)$ & $\mathrm{Cu}(1)$ & $P(2)$ & $C(13)$ & $74.4(2)$ \\
\hline$P(1)$ & $\mathrm{Cu}(1)$ & $P(2)$ & $C(19)$ & $-150.4(2)$ & $P(1)$ & $\mathrm{Cu}(1)$ & $P(2)$ & $C(30)$ & $-32.5(2)$ \\
\hline $\mathrm{Cl}(2)$ & $\mathrm{Cu}(2)$ & $\mathrm{Cl}(1)$ & $\mathrm{Cu}(1)$ & $-18.12(5)$ & $P(3)$ & $\mathrm{Cu}(2)$ & $\mathrm{Cl}(1)$ & $\mathrm{Cu}(1)$ & $103.99(5)$ \\
\hline$P(4)$ & $\mathrm{Cu}(2)$ & $\mathrm{Cl}(1)$ & $\mathrm{Cu}(1)$ & $-142.30(5)$ & $\mathrm{Cl}(1)$ & $\mathrm{Cu}(2)$ & $\mathrm{Cl}(2)$ & $\mathrm{Cu}(1)$ & $18.70(5)$ \\
\hline$P(3)$ & $\mathrm{Cu}(2)$ & $\mathrm{Cl}(2)$ & $\mathrm{Cu}(1)$ & $-112.08(4)$ & $P(4)$ & $\mathrm{Cu}(2)$ & $\mathrm{Cl}(2)$ & $\mathrm{Cu}(1)$ & $149.39(4)$ \\
\hline $\mathrm{Cl}(1)$ & $\mathrm{Cu}(2)$ & $P(3)^{\prime}$ & $C(31)$ & $-96.3(2)$ & $\mathrm{Cl}(1)$ & $\mathrm{Cu}(2)$ & $P(3)^{\prime}$ & $C(37)$ & $37.4(2)$ \\
\hline $\mathrm{Cl}(1)$ & $\mathrm{Cu}(2)$ & $P(3)$ & $C(60)$ & $149.6(1)$ & $\mathrm{Cl}(2)$ & $\mathrm{Cu}(2)$ & $P(3)$ & $C(31)$ & $22.2(2)$ \\
\hline $\mathrm{Cl}(2)$ & $\mathrm{Cu}(2)$ & $P(3)$ & $C(37)$ & $155.9(2)$ & $\mathrm{Cl}(2)$ & $\mathrm{Cu}(2)$ & $P(3)$ & $C(60)$ & $-91.9(2)$ \\
\hline$P(4)$ & $\mathrm{Cu}(2)$ & $P(3)$ & $C(31)$ & $135.1(2)$ & $P(4)$ & $\mathrm{Cu}(2)$ & $P(3)$ & C(37) & $-91.2(2)$ \\
\hline$P(4)$ & $\mathrm{Cu}(2)$ & $P(3)$ & $C(60)$ & $21.1(2)$ & $\mathrm{Cl}(1)$ & $\mathrm{Cu}(2)$ & $P(4)$ & $C(43)$ & $-36.3(2)$ \\
\hline $\mathrm{Cl}(1)$ & $\mathrm{Cu}(2)$ & $P(4)$ & C(49) & $92.6(2)$ & $\mathrm{Cl}(1)$ & $\mathrm{Cu}(2)$ & $P(4)$ & C(55) & $-150.2(1)$ \\
\hline $\mathrm{Cl}(2)$ & $\mathrm{Cu}(2)$ & $P(4)$ & $C(43)$ & $-155.8(2)$ & $\mathrm{Cl}(2)$ & $\mathrm{Cu}(2)$ & $P(4)$ & $C(49)$ & $-26.9(2)$ \\
\hline $\mathrm{Cl}(2)$ & $\mathrm{Cu}(2)$ & $P(4)$ & C(55) & $90.3(2)$ & $P(3)$ & $\mathrm{Cu}(2)$ & $P(4)$ & $C(43)$ & $93.1(2)$ \\
\hline$P(3)$ & $\mathrm{Cu}(2)$ & $P(4)$ & $C(49)$ & $-138.0(2)$ & $P(3)$ & $\mathrm{Cu}(2)$ & $P(4)$ & $C(55)$ & $-20.8(2)$ \\
\hline $\mathrm{Cu}(1)$ & $P(1)$ & $C(1)$ & $C(2)$ & $-55.8(4)$ & $\mathrm{Cu}(1)$ & $P(1)$ & $C(1)$ & $C(6)$ & $119.4(4)$ \\
\hline$C(7)$ & $P(1)$ & $C(1)$ & $C(2)$ & $84.8(4)$ & $C(7)$ & $P(1)$ & $C(1)$ & $C(6)$ & $-100.1(4)$ \\
\hline$C(25)$ & $P(1)$ & $C(1)$ & $C(2)$ & $-165.8(3)$ & $C(25)$ & $P(1)$ & $C(1)$ & $C(6)$ & $9.4(5)$ \\
\hline $\mathrm{Cu}(1)$ & $P(1)$ & $C(7)$ & $C(8)$ & $-28.8(5)$ & $\mathrm{Cu}(1)$ & $P(1)$ & $\mathrm{C}(7)$ & $C(12)$ & $150.3(3)$ \\
\hline$C(1)$ & $P(1)$ & $C(7)$ & $C(8)$ & $-164.0(4)$ & $C(1)$ & $P(1)$ & $C(7)$ & $C(12)$ & $15.2(5)$ \\
\hline$C(25)$ & $P(1)$ & $C(7)$ & $C(8)$ & $87.0(4)$ & $C(25)$ & $P(1)$ & $C(7)$ & $C(12)$ & $-93.8(4)$ \\
\hline $\mathrm{Cu}(1)$ & $P(1)$ & $C(25)$ & $C(26)$ & 157.6(3) & $\mathrm{Cu}(1)$ & $P(1)$ & $C(25)$ & $C(30)$ & $-20.7(4)$ \\
\hline$C(1)$ & $P(1)$ & $C(25)$ & $C(26)$ & $-83.3(4)$ & $C(1)$ & $P(1)$ & $C(25)$ & $C(30)$ & $98.3(3)$ \\
\hline$C(7)$ & $P(1)$ & $C(25)$ & $C(26)$ & $26.6(4)$ & $\mathrm{C}(7)$ & $P(1)$ & $C(25)$ & $C(30)$ & $-151.8(3)$ \\
\hline $\mathrm{Cu}(1)$ & $P(2)$ & $C(13)$ & $C(14)$ & $20.8(5)$ & $\mathrm{Cu}(1)$ & $P(2)$ & $C(13)$ & $C(18)$ & $-151.4(4)$ \\
\hline$C(19)$ & $P(2)$ & $C(13)$ & $C(14)$ & $-122.7(4)$ & $C(19)$ & $P(2)$ & $C(13)$ & $C(18)$ & $65.0(5)$ \\
\hline$C(30)$ & $P(2)$ & $C(13)$ & $C(14)$ & $127.6(4)$ & $C(30)$ & $P(2)$ & $C(13)$ & $C(18)$ & $-44.6(5)$ \\
\hline $\mathrm{Cu}(1)$ & $P(2)$ & $C(19)$ & $C(20)$ & $-127.3(4)$ & $\mathrm{Cu}(1)$ & $P(2)$ & $C(19)$ & $C(24)$ & $47.3(5)$ \\
\hline
\end{tabular}

Table 8. Torsion angles $\left({ }^{\circ}\right)$-- continued

$\begin{array}{llllllllll}\text { atom1 } & \text { atom2 } & \text { atom3 } & \text { atom4 } & \text { angle } & \text { atom1 } & \text { atom2 } & \text { atom3 } & \text { atom4 } & \text { angle } \\ \mathrm{C}(13) & \mathrm{P}(2) & \mathrm{C}(19) & \mathrm{C}(20) & 10.5(5) & \mathrm{C}(13) & \mathrm{P}(2) & \mathrm{C}(19) & \mathrm{C}(24) & -174.9(4) \\ \mathrm{C}(30) & \mathrm{P}(2) & \mathrm{C}(19) & \mathrm{C}(20) & 116.9(4) & \mathrm{C}(30) & \mathrm{P}(2) & \mathrm{C}(19) & \mathrm{C}(24) & -68.5(4) \\ \mathrm{Cu}(1) & \mathrm{P}(2) & \mathrm{C}(30) & \mathrm{C}(25) & 29.7(4) & \mathrm{Cu}(1) & \mathrm{P}(2) & \mathrm{C}(30) & \mathrm{C}(29) & -153.8(4) \\ \mathrm{C}(13) & \mathrm{P}(2) & \mathrm{C}(30) & \mathrm{C}(25) & -87.5(3) & \mathrm{C}(13) & \mathrm{P}(2) & \mathrm{C}(30) & \mathrm{C}(29) & 89.0(4) \\ \mathrm{C}(19) & \mathrm{P}(2) & \mathrm{C}(30) & \mathrm{C}(25) & 162.0(3) & \mathrm{C}(19) & \mathrm{P}(2) & \mathrm{C}(30) & \mathrm{C}(29) & -21.5(4) \\ \mathrm{Cu}(2) & \mathrm{P}(3) & \mathrm{C}(31) & \mathrm{C}(32) & -52.8(4) & \mathrm{Cu}(2) & \mathrm{P}(3) & \mathrm{C}(31) & \mathrm{C}(36) & 121.6(4) \\ \mathrm{C}(37) & \mathrm{P}(3) & \mathrm{C}(31) & \mathrm{C}(32) & 167.8(3) & \mathrm{C}(37) & \mathrm{P}(3) & \mathrm{C}(31) & \mathrm{C}(36) & -17.8(4) \\ \mathrm{C}(60) & \mathrm{P}(3) & \mathrm{C}(31) & \mathrm{C}(32) & 60.8(4) & \mathrm{C}(60) & \mathrm{P}(3) & \mathrm{C}(31) & \mathrm{C}(36) & -124.8(4) \\ \mathrm{Cu}(2) & \mathrm{P}(3) & \mathrm{C}(37) & \mathrm{C}(38) & 169.0(3) & \mathrm{Cu}(2) & \mathrm{P}(3) & \mathrm{C}(37) & \mathrm{C}(42) & -12.8(4) \\ \mathrm{C}(31) & \mathrm{P}(3) & \mathrm{C}(37) & \mathrm{C}(38) & -50.8(4) & \mathrm{C}(31) & \mathrm{P}(3) & \mathrm{C}(37) & \mathrm{C}(42) & 127.5(4) \\ \mathrm{C}(60) & \mathrm{P}(3) & \mathrm{C}(37) & \mathrm{C}(38) & 56.8(4) & \mathrm{C}(60) & \mathrm{P}(3) & \mathrm{C}(37) & \mathrm{C}(42) & -124.9(4)\end{array}$




\begin{tabular}{|c|c|c|c|c|c|c|c|c|c|}
\hline $\mathrm{Cu}(2)$ & $P(3)$ & $C(60)$ & $C(55)$ & $-17.6(4)$ & $\mathrm{Cu}(2)$ & $P(3)$ & $C(60)$ & $C(59)$ & $162.6(4)$ \\
\hline$C(31)$ & $P(3)$ & $C(60)$ & $C(55)$ & $-144.3(3)$ & $C(31)$ & $P(3)$ & $C(60)$ & C(59) & $35.9(4)$ \\
\hline$C(37)$ & $P(3)$ & $C(60)$ & $C(55)$ & $106.8(4)$ & $C(37)$ & $P(3)$ & $C(60)$ & C(59) & $-72.9(4)$ \\
\hline $\mathrm{Cu}(2)$ & $P(4)$ & $C(43)$ & $C(44)$ & $39.1(5)$ & $\mathrm{Cu}(2)$ & $P(4)$ & $C(43)$ & $C(48)$ & $-143.1(4)$ \\
\hline$C(49)$ & $P(4)$ & $C(43)$ & $C(44)$ & $-98.3(4)$ & $C(49)$ & $P(4)$ & C(43) & $C(48)$ & $79.5(5)$ \\
\hline$C(55)$ & $P(4)$ & $C(43)$ & $C(44)$ & $151.8(4)$ & $C(55)$ & $P(4)$ & $C(43)$ & $C(48)$ & $-30.4(5)$ \\
\hline $\mathrm{Cu}(2)$ & $P(4)$ & $C(49)$ & $C(50)$ & $17.7(5)$ & $\mathrm{Cu}(2)$ & $P(4)$ & $C(49)$ & $C(54)$ & $-162.2(4)$ \\
\hline$C(43)$ & $P(4)$ & $C(49)$ & $C(50)$ & $151.4(4)$ & $C(43)$ & $P(4)$ & $C(49)$ & $C(54)$ & $-28.5(5)$ \\
\hline C(55) & $P(4)$ & C(49) & $C(50)$ & $-98.6(4)$ & $C(55)$ & $P(4)$ & $C(49)$ & C(54) & $81.5(5)$ \\
\hline $\mathrm{Cu}(2)$ & $P(4)$ & $C(55)$ & $C(56)$ & $-163.0(3)$ & $\mathrm{Cu}(2)$ & $P(4)$ & $C(55)$ & $C(60)$ & $15.8(4)$ \\
\hline$C(43)$ & $P(4)$ & $C(55)$ & $C(56)$ & $75.5(4)$ & $C(43)$ & $P(4)$ & C(55) & $C(60)$ & $-105.8(3)$ \\
\hline$C(49)$ & $P(4)$ & $C(55)$ & $C(56)$ & $-33.9(4)$ & $\mathrm{C}(49)$ & $P(4)$ & C(55) & $C(60)$ & $144.9(3)$ \\
\hline$P(1)$ & $C(1)$ & $C(2)$ & $C(3)$ & $175.3(5)$ & $C(6)$ & $C(1)$ & $C(2)$ & $C(3)$ & $-0.1(6)$ \\
\hline$P(1)$ & $C(1)$ & $C(6)$ & C(5) & $-174.1(5)$ & $C(2)$ & $C(1)$ & $C(6)$ & $C(5)$ & $0.9(8)$ \\
\hline$C(1)$ & $C(2)$ & $C(3)$ & $C(4)$ & $-0.5(8)$ & $C(2)$ & $C(3)$ & $C(4)$ & $C(5)$ & $0.3(7)$ \\
\hline$C(3)$ & $C(4)$ & $C(5)$ & $C(6)$ & $0.5(8)$ & $C(4)$ & $C(5)$ & $C(6)$ & $C(1)$ & $-1.1(9)$ \\
\hline$P(1)$ & $C(7)$ & $C(8)$ & $\mathrm{C}(9)$ & $178.2(5)$ & $C(12)$ & $C(7)$ & $C(8)$ & $\mathrm{C}(9)$ & $-1.0(8)$ \\
\hline$P(1)$ & $C(7)$ & $C(12)$ & $C(11)$ & $-177.5(4)$ & $\mathrm{C}(8)$ & $C(7)$ & $C(12)$ & $C(11)$ & $1.7(7)$ \\
\hline$C(7)$ & $C(8)$ & C(9) & $C(10)$ & $-0.4(8)$ & $C(8)$ & $\mathrm{C}(9)$ & $C(10)$ & $C(11)$ & $1.1(9)$ \\
\hline$C(9)$ & $C(10)$ & $C(11)$ & $C(12)$ & $-0.4(8)$ & $C(10)$ & $C(11)$ & $C(12)$ & $C(7)$ & $-1.0(8)$ \\
\hline$P(2)$ & $C(13)$ & $C(14)$ & $C(15)$ & $-172.3(4)$ & $C(18)$ & $C(13)$ & $C(14)$ & $C(15)$ & $0.3(7)$ \\
\hline$P(2)$ & $C(13)$ & $C(18)$ & $C(17)$ & $173.3(5)$ & $C(14)$ & $C(13)$ & $C(18)$ & $C(17)$ & $1.0(8)$ \\
\hline$C(13)$ & $C(14)$ & $C(15)$ & $C(16)$ & $0.2(6)$ & $C(14)$ & $C(15)$ & $C(16)$ & $C(17)$ & $-2.0(9)$ \\
\hline$C(15)$ & $C(16)$ & $C(17)$ & $C(18)$ & $3.3(9)$ & $C(16)$ & $C(17)$ & $C(18)$ & $C(13)$ & $-2.8(9)$ \\
\hline$P(2)$ & $C(19)$ & $C(20)$ & $C(21)$ & $172.9(5)$ & $C(24)$ & $C(19)$ & $C(20)$ & $C(21)$ & $-1.7(8)$ \\
\hline$P(2)$ & $C(19)$ & $C(24)$ & $C(23)$ & $-174.2(5)$ & $C(20)$ & $C(19)$ & $C(24)$ & $C(23)$ & $0.9(8)$ \\
\hline
\end{tabular}

Table 8. Torsion angles $\left({ }^{0}\right)$-- continued

$\begin{array}{llllllllll}\text { atom1 } & \text { atom2 } & \text { atom3 } & \text { atom4 } & \text { angle } & \text { atom1 } & \text { atom2 } & \text { atom3 } & \text { atom4 } & \text { angle } \\ \mathrm{C}(19) & \mathrm{C}(20) & \mathrm{C}(21) & \mathrm{C}(22) & 1(1) & \mathrm{C}(20) & \mathrm{C}(21) & \mathrm{C}(22) & \mathrm{C}(23) & 0(1) \\ \mathrm{C}(21) & \mathrm{C}(22) & \mathrm{C}(23) & \mathrm{C}(24) & -0.1(8) & \mathrm{C}(22) & \mathrm{C}(23) & \mathrm{C}(24) & \mathrm{C}(19) & 0.0(8) \\ \mathrm{P}(1) & \mathrm{C}(25) & \mathrm{C}(26) & \mathrm{C}(27) & -178.6(4) & \mathrm{C}(30) & \mathrm{C}(25) & \mathrm{C}(26) & \mathrm{C}(27) & -0.3(5) \\ \mathrm{P}(1) & \mathrm{C}(25) & \mathrm{C}(30) & \mathrm{P}(2) & -6.2(5) & \mathrm{P}(1) & \mathrm{C}(25) & \mathrm{C}(30) & \mathrm{C}(29) & 177.1(4) \\ \mathrm{C}(26) & \mathrm{C}(25) & \mathrm{C}(30) & \mathrm{P}(2) & 175.4(3) & \mathrm{C}(26) & \mathrm{C}(25) & \mathrm{C}(30) & \mathrm{C}(29) & -1.3(6) \\ \mathrm{C}(25) & \mathrm{C}(26) & \mathrm{C}(27) & \mathrm{C}(28) & 2.0(7) & \mathrm{C}(26) & \mathrm{C}(27) & \mathrm{C}(28) & \mathrm{C}(29) & -2.2(7) \\ \mathrm{C}(27) & \mathrm{C}(28) & \mathrm{C}(29) & \mathrm{C}(30) & 0.6(7) & \mathrm{C}(28) & \mathrm{C}(29) & \mathrm{C}(30) & \mathrm{P}(2) & -175.4(3) \\ \mathrm{C}(28) & \mathrm{C}(29) & \mathrm{C}(30) & \mathrm{C}(25) & 1.1(7) & \mathrm{P}(3) & \mathrm{C}(31) & \mathrm{C}(32) & \mathrm{C}(33) & 177.9(4) \\ \mathrm{C}(36) & \mathrm{C}(31) & \mathrm{C}(32) & \mathrm{C}(33) & 3.1(7) & \mathrm{P}(3) & \mathrm{C}(31) & \mathrm{C}(36) & \mathrm{C}(35) & -176.3(5) \\ \mathrm{C}(32) & \mathrm{C}(31) & \mathrm{C}(36) & \mathrm{C}(35) & -1.9(7) & \mathrm{C}(31) & \mathrm{C}(32) & \mathrm{C}(33) & \mathrm{C}(34) & -1.8(8) \\ \mathrm{C}(32) & \mathrm{C}(33) & \mathrm{C}(34) & \mathrm{C}(35) & 0(1) & \mathrm{C}(33) & \mathrm{C}(34) & \mathrm{C}(35) & \mathrm{C}(36) & 2(1) \\ \mathrm{C}(34) & \mathrm{C}(35) & \mathrm{C}(36) & \mathrm{C}(31) & -0.8(9) & \mathrm{P}(3) & \mathrm{C}(37) & \mathrm{C}(38) & \mathrm{C}(39) & 179.1(4) \\ \mathrm{C}(42) & \mathrm{C}(37) & \mathrm{C}(38) & \mathrm{C}(39) & 0.8(7) & \mathrm{P}(3) & \mathrm{C}(37) & \mathrm{C}(42) & \mathrm{C}(41) & -179.0(4) \\ \mathrm{C}(38) & \mathrm{C}(37) & \mathrm{C}(42) & \mathrm{C}(41) & -0.6(7) & \mathrm{C}(37) & \mathrm{C}(38) & \mathrm{C}(39) & \mathrm{C}(40) & -1.2(8) \\ \mathrm{C}(38) & \mathrm{C}(39) & \mathrm{C}(40) & \mathrm{C}(41) & 1.3(8) & \mathrm{C}(39) & \mathrm{C}(40) & \mathrm{C}(41) & \mathrm{C}(42) & -1.1(9) \\ \mathrm{C}(40) & \mathrm{C}(41) & \mathrm{C}(42) & \mathrm{C}(37) & 0.8(8) & \mathrm{P}(4) & \mathrm{C}(43) & \mathrm{C}(44) & \mathrm{C}(45) & 179.3(5) \\ \mathrm{C}(48) & \mathrm{C}(43) & \mathrm{C}(44) & \mathrm{C}(45) & 1.3(8) & \mathrm{P}(4) & \mathrm{C}(43) & \mathrm{C}(48) & \mathrm{C}(47) & -177.8(5) \\ \mathrm{C}(44) & \mathrm{C}(43) & \mathrm{C}(48) & \mathrm{C}(47) & -0.1(7) & \mathrm{C}(43) & \mathrm{C}(44) & \mathrm{C}(45) & \mathrm{C}(46) & 0.5(9) \\ \mathrm{C}(44) & \mathrm{C}(45) & \mathrm{C}(46) & \mathrm{C}(47) & -3.6(9) & \mathrm{C}(45) & \mathrm{C}(46) & \mathrm{C}(47) & \mathrm{C}(48) & 4.8(9) \\ \mathrm{C}(46) & \mathrm{C}(47) & \mathrm{C}(48) & \mathrm{C}(43) & -3.0(9) & \mathrm{P}(4) & \mathrm{C}(49) & \mathrm{C}(50) & \mathrm{C}(51) & -178.8(4) \\ \mathrm{C}(54) & \mathrm{C}(49) & \mathrm{C}(50) & \mathrm{C}(51) & 1.1(7) & \mathrm{P}(4) & \mathrm{C}(49) & \mathrm{C}(54) & \mathrm{C}(53) & 179.9(4)\end{array}$




$\begin{array}{llllllllll}\mathrm{C}(50) & \mathrm{C}(49) & \mathrm{C}(54) & \mathrm{C}(53) & -0.0(7) & \mathrm{C}(49) & \mathrm{C}(50) & \mathrm{C}(51) & \mathrm{C}(52) & -1.6(9) \\ \mathrm{C}(50) & \mathrm{C}(51) & \mathrm{C}(52) & \mathrm{C}(53) & 1.0(9) & \mathrm{C}(51) & \mathrm{C}(52) & \mathrm{C}(53) & \mathrm{C}(54) & 0(1) \\ \mathrm{C}(52) & \mathrm{C}(53) & \mathrm{C}(54) & \mathrm{C}(49) & -0.5(9) & \mathrm{P}(4) & \mathrm{C}(55) & \mathrm{C}(56) & \mathrm{C}(57) & -179.4(4) \\ \mathrm{C}(60) & \mathrm{C}(55) & \mathrm{C}(56) & \mathrm{C}(57) & 1.9(7) & \mathrm{P}(4) & \mathrm{C}(55) & \mathrm{C}(60) & \mathrm{P}(3) & 1.2(5) \\ \mathrm{P}(4) & \mathrm{C}(55) & \mathrm{C}(60) & \mathrm{C}(59) & -179.0(4) & \mathrm{C}(56) & \mathrm{C}(55) & \mathrm{C}(60) & \mathrm{P}(3) & -180.0(3) \\ \mathrm{C}(56) & \mathrm{C}(55) & \mathrm{C}(60) & \mathrm{C}(59) & -0.2(6) & \mathrm{C}(55) & \mathrm{C}(56) & \mathrm{C}(57) & \mathrm{C}(58) & -2.5(7) \\ \mathrm{C}(56) & \mathrm{C}(57) & \mathrm{C}(58) & \mathrm{C}(59) & 1.5(8) & \mathrm{C}(57) & \mathrm{C}(58) & \mathrm{C}(59) & \mathrm{C}(60) & 0.1(6) \\ \mathrm{C}(58) & \mathrm{C}(59) & \mathrm{C}(60) & \mathrm{P}(3) & 179.0(4) & \mathrm{C}(58) & \mathrm{C}(59) & \mathrm{C}(60) & \mathrm{C}(55) & -0.7(7)\end{array}$

The sign is positive if when looking from atom 2 to atom 3 a clock-wise motion of atom 1 would superimpose it on atom 4 . 


\title{
$\mathrm{Cul}(\mathrm{dppb}) \mathrm{PPh}_{3}$ (4)
}

\section{Table S6.}

\section{EXPERIMENTAL DETAILS}

A. Crystal Data

\author{
Empirical Formula \\ Formula Weight \\ Crystal Color, Habit \\ Crystal Dimensions \\ Crystal System \\ Lattice Type \\ Indexing Images \\ Detector Position \\ Pixel Size \\ Lattice Parameters
}

Space Group

$Z$ value

$D_{\text {calc }}$

$\mathrm{F}_{000}$

$\mu(\mathrm{MoK} \alpha)$

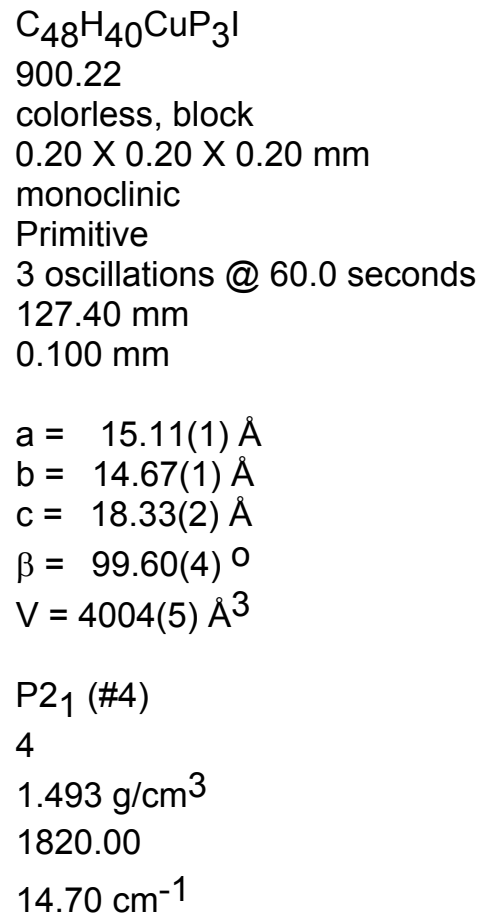

B. Intensity Measurements

Diffractometer

Radiation

Detector Aperture

Data Images

$\omega$ oscillation Range $(\chi=45.0, \phi=0.0)$

Exposure Rate

$\omega$ oscillation Range ( $\chi=45.0, \phi=210.0)$

Exposure Rate

Detector Position

Pixel Size

$2 \theta$ max

No. of Reflections Measured
Rigaku RAXIS-RAPID

$\operatorname{MoK} \alpha(\lambda=0.71075 \AA)$

graphite monochromated

$280 \mathrm{~mm} \times 256 \mathrm{~mm}$

55 exposures

$130.0-190.0^{\circ}$

$30.0 \mathrm{sec} . / 0$

$0.0-160.0^{\circ}$

$30.0 \mathrm{sec} . / \mathrm{O}$

$127.40 \mathrm{~mm}$

$0.100 \mathrm{~mm}$

55.0

Total: 35524

Unique: $35498\left(R_{\text {int }}=0.045\right)$ 
Corrections

Lorentz-polarization

Absorption

(trans. factors: $0.4919-1.0000$ )

C. Structure Solution and Refinement

Structure Solution

Refinement

Function Minimized

Least Squares Weights

$2 \theta$ max cutoff

Anomalous Dispersion

No. Observations (All reflections)

No. Variables

Reflection/Parameter Ratio

Residuals: $R$ (All reflections)

Residuals: R1 (I>2.00\%(I))

Residuals: wR2 (All reflections)

Goodness of Fit Indicator

Flack Parameter

Max Shift/Error in Final Cycle

Maximum peak in Final Diff. Map

Minimum peak in Final Diff. Map

Table 4. Bond lengths $(\AA)$

$\begin{array}{lll}\text { atom } & \text { atom } & \text { distance } \\ \mathrm{I}(1) & \mathrm{Cu}(1) & 2.5975(4) \\ \mathrm{Cu}(1) & \mathrm{P}(1) & 2.2609(9) \\ \mathrm{Cu}(1) & \mathrm{P}(3) & 2.271(1) \\ \mathrm{Cu}(2) & \mathrm{P}(5) & 2.2617(9) \\ \mathrm{P}(1) & \mathrm{C}(31) & 1.825(3) \\ \mathrm{P}(1) & \mathrm{C}(43) & 1.826(3) \\ \mathrm{P}(2) & \mathrm{C}(7) & 1.839(3) \\ \mathrm{P}(3) & \mathrm{C}(6) & 1.840(3) \\ \mathrm{P}(3) & \mathrm{C}(25) & 1.840(3) \\ \mathrm{P}(4) & \mathrm{C}(85) & 1.821(3) \\ \mathrm{P}(5) & \mathrm{C}(49) & 1.828(3) \\ \mathrm{P}(5) & \mathrm{C}(61) & 1.821(3) \\ \mathrm{P}(6) & \mathrm{C}(67) & 1.826(3) \\ \mathrm{C}(1) & \mathrm{C}(2) & 1.396(5) \\ \mathrm{C}(2) & \mathrm{C}(3) & 1.384(5) \\ \mathrm{C}(4) & \mathrm{C}(5) & 1.377(5) \\ \mathrm{C}(7) & \mathrm{C}(8) & 1.389(5) \\ \mathrm{C}(8) & \mathrm{C}(9) & 1.414(4) \\ \mathrm{C}(10) & \mathrm{C}(11) & 1.382(5) \\ \mathrm{C}(13) & \mathrm{C}(14) & 1.389(4) \\ \mathrm{C}(14) & \mathrm{C}(15) & 1.380(5) \\ \mathrm{C}(16) & \mathrm{C}(17) & 1.377(5) \\ \mathrm{C}(19) & \mathrm{C}(20) & 1.384(5) \\ \mathrm{C}(20) & \mathrm{C}(21) & 1.398(5)\end{array}$

Direct Methods (SIR92)

Full-matrix least-squares on $\mathrm{F}^{2}$

$\Sigma \mathrm{w}\left(\mathrm{Fo}^{2}-\mathrm{Fc}^{2}\right)^{2}$

$1 /\left[0.0002 \mathrm{Fo}^{2}+0.5000 \sigma\left(\mathrm{Fo}^{2}\right)\right] /\left(4 \mathrm{Fo}^{2}\right)$

$55.0^{\circ}$

All non-hydrogen atoms

35498

1034

34.33

0.044

0.037

0.082

1.269

$0.029(6)$

0.000

$5.36 \mathrm{e}^{-/} / \AA^{3}$

$-3.69 e^{-} / \AA^{3}$

$\begin{array}{lll}\text { atom } & \text { atom } & \text { distance } \\ \mathrm{l}(2) & \mathrm{Cu}(2) & 2.6409(4) \\ \mathrm{Cu}(1) & \mathrm{P}(2) & 2.278(1) \\ \mathrm{Cu}(2) & \mathrm{P}(4) & 2.237(1) \\ \mathrm{Cu}(2) & \mathrm{P}(6) & 2.2774(8) \\ \mathrm{P}(1) & \mathrm{C}(37) & 1.829(3) \\ \mathrm{P}(2) & \mathrm{C}(1) & 1.817(3) \\ \mathrm{P}(2) & \mathrm{C}(13) & 1.826(3) \\ \mathrm{P}(3) & \mathrm{C}(19) & 1.825(3) \\ \mathrm{P}(4) & \mathrm{C}(79) & 1.821(3) \\ \mathrm{P}(4) & \mathrm{C}(91) & 1.834(3) \\ \mathrm{P}(5) & \mathrm{C}(55) & 1.821(3) \\ \mathrm{P}(6) & \mathrm{C}(54) & 1.850(3) \\ \mathrm{P}(6) & \mathrm{C}(73) & 1.815(3) \\ \mathrm{C}(1) & \mathrm{C}(6) & 1.408(4) \\ \mathrm{C}(3) & \mathrm{C}(4) & 1.381(5) \\ \mathrm{C}(5) & \mathrm{C}(6) & 1.395(4) \\ \mathrm{C}(7) & \mathrm{C}(12) & 1.386(5) \\ \mathrm{C}(9) & \mathrm{C}(10) & 1.370(5) \\ \mathrm{C}(11) & \mathrm{C}(12) & 1.395(4) \\ \mathrm{C}(13) & \mathrm{C}(18) & 1.392(5) \\ \mathrm{C}(15) & \mathrm{C}(16) & 1.382(5) \\ \mathrm{C}(17) & \mathrm{C}(18) & 1.381(5) \\ \mathrm{C}(19) & \mathrm{C}(24) & 1.391(4) \\ \mathrm{C}(21) & \mathrm{C}(22) & 1.360(5)\end{array}$




$\begin{array}{llllll}\mathrm{C}(22) & \mathrm{C}(23) & 1.372(5) & \mathrm{C}(23) & \mathrm{C}(24) & 1.384(5) \\ \mathrm{C}(25) & \mathrm{C}(26) & 1.363(5) & \mathrm{C}(25) & \mathrm{C}(30) & 1.397(5) \\ \mathrm{C}(26) & \mathrm{C}(27) & 1.406(5) & \mathrm{C}(27) & \mathrm{C}(28) & 1.377(6) \\ \mathrm{C}(28) & \mathrm{C}(29) & 1.372(6) & \mathrm{C}(29) & \mathrm{C}(30) & 1.382(4) \\ \mathrm{C}(31) & \mathrm{C}(32) & 1.365(5) & \mathrm{C}(31) & \mathrm{C}(36) & 1.398(5) \\ \mathrm{C}(32) & \mathrm{C}(33) & 1.392(5) & \mathrm{C}(33) & \mathrm{C}(32) & 1.392(5) \\ \mathrm{C}(33) & \mathrm{C}(34) & 1.343(6) & \mathrm{C}(34) & \mathrm{C}(33) & 1.343(6) \\ \mathrm{C}(34) & \mathrm{C}(35) & 1.364(6) & \mathrm{C}(35) & \mathrm{C}(36) & 1.362(5) \\ \mathrm{C}(37) & \mathrm{C}(38) & 1.380(5) & \mathrm{C}(37) & \mathrm{C}(42) & 1.373(5) \\ \mathrm{C}(38) & \mathrm{C}(39) & 1.381(6) & \mathrm{C}(39) & \mathrm{C}(40) & 1.362(6) \\ \mathrm{C}(40) & \mathrm{C}(41) & 1.351(5) & \mathrm{C}(41) & \mathrm{C}(42) & 1.401(5) \\ \mathrm{C}(43) & \mathrm{C}(44) & 1.391(5) & \mathrm{C}(43) & \mathrm{C}(48) & 1.396(5) \\ \mathrm{C}(44) & \mathrm{C}(45) & 1.378(5) & \mathrm{C}(45) & \mathrm{C}(46) & 1.386(5)\end{array}$

Table 4. Bond lengths $(\AA) \quad$-- continued

$\begin{array}{llllll}\text { atom } & \text { atom } & \text { distance } & \text { atom } & \text { atom } & \text { distance } \\ \mathrm{C}(46) & \mathrm{C}(47) & 1.373(5) & \mathrm{C}(47) & \mathrm{C}(48) & 1.386(5) \\ \mathrm{C}(49) & \mathrm{C}(50) & 1.383(5) & \mathrm{C}(49) & \mathrm{C}(54) & 1.417(4) \\ \mathrm{C}(50) & \mathrm{C}(51) & 1.405(5) & \mathrm{C}(51) & \mathrm{C}(52) & 1.391(5) \\ \mathrm{C}(52) & \mathrm{C}(53) & 1.371(5) & \mathrm{C}(53) & \mathrm{C}(54) & 1.391(5) \\ \mathrm{C}(55) & \mathrm{C}(56) & 1.378(4) & \mathrm{C}(55) & \mathrm{C}(60) & 1.401(4) \\ \mathrm{C}(56) & \mathrm{C}(57) & 1.385(4) & \mathrm{C}(57) & \mathrm{C}(58) & 1.395(5) \\ \mathrm{C}(58) & \mathrm{C}(59) & 1.372(5) & \mathrm{C}(59) & \mathrm{C}(60) & 1.395(5) \\ \mathrm{C}(61) & \mathrm{C}(62) & 1.399(5) & \mathrm{C}(61) & \mathrm{C}(66) & 1.388(4) \\ \mathrm{C}(62) & \mathrm{C}(63) & 1.371(5) & \mathrm{C}(63) & \mathrm{C}(64) & 1.389(5) \\ \mathrm{C}(64) & \mathrm{C}(65) & 1.367(5) & \mathrm{C}(65) & \mathrm{C}(66) & 1.395(5) \\ \mathrm{C}(67) & \mathrm{C}(68) & 1.391(5) & \mathrm{C}(67) & \mathrm{C}(72) & 1.399(5) \\ \mathrm{C}(68) & \mathrm{C}(69) & 1.384(5) & \mathrm{C}(69) & \mathrm{C}(70) & 1.369(5) \\ \mathrm{C}(70) & \mathrm{C}(71) & 1.382(5) & \mathrm{C}(71) & \mathrm{C}(72) & 1.381(5) \\ \mathrm{C}(73) & \mathrm{C}(74) & 1.397(5) & \mathrm{C}(73) & \mathrm{C}(78) & 1.386(5) \\ \mathrm{C}(74) & \mathrm{C}(75) & 1.389(5) & \mathrm{C}(75) & \mathrm{C}(76) & 1.382(5) \\ \mathrm{C}(76) & \mathrm{C}(77) & 1.363(5) & \mathrm{C}(77) & \mathrm{C}(78) & 1.382(5) \\ \mathrm{C}(79) & \mathrm{C}(80) & 1.389(4) & \mathrm{C}(79) & \mathrm{C}(84) & 1.392(5) \\ \mathrm{C}(80) & \mathrm{C}(81) & 1.386(5) & \mathrm{C}(81) & \mathrm{C}(82) & 1.369(5) \\ \mathrm{C}(82) & \mathrm{C}(83) & 1.391(5) & \mathrm{C}(83) & \mathrm{C}(84) & 1.387(5) \\ \mathrm{C}(85) & \mathrm{C}(86) & 1.380(5) & \mathrm{C}(85) & \mathrm{C}(90) & 1.398(5) \\ \mathrm{C}(86) & \mathrm{C}(87) & 1.382(5) & \mathrm{C}(87) & \mathrm{C}(88) & 1.380(5) \\ \mathrm{C}(88) & \mathrm{C}(89) & 1.362(6) & \mathrm{C}(89) & \mathrm{C}(90) & 1.398(5) \\ \mathrm{C}(91) & \mathrm{C}(92) & 1.384(5) & \mathrm{C}(91) & \mathrm{C}(96) & 1.392(4) \\ \mathrm{C}(92) & \mathrm{C}(93) & 1.376(5) & \mathrm{C}(93) & \mathrm{C}(94) & 1.373(5) \\ \mathrm{C}(94) & \mathrm{C}(95) & 1.378(6) & \mathrm{C}(95) & \mathrm{C}(96) & 1.397(5) \\ & & & & \end{array}$

Table 6. Bond angles $\left(^{\circ}\right)$

$\begin{array}{llllllll}\text { atom } & \text { atom } & \text { atom } & \text { angle } & \text { atom } & \text { atom } & \text { atom } & \text { angle } \\ \mathrm{I}(1) & \mathrm{Cu}(1) & \mathrm{P}(1) & 101.59(3) & \mathrm{I}(1) & \mathrm{Cu}(1) & \mathrm{P}(2) & 116.34(2) \\ \mathrm{I}(1) & \mathrm{Cu}(1) & \mathrm{P}(3) & 111.60(3) & \mathrm{I}(2) & \mathrm{Cu}(2) & \mathrm{P}(4) & 111.79(2) \\ \mathrm{I}(2) & \mathrm{Cu}(2) & \mathrm{P}(5) & 98.76(3) & \mathrm{I}(2) & \mathrm{Cu}(2) & \mathrm{P}(6) & 100.23(3) \\ \mathrm{P}(2) & \mathrm{Cu}(1) & \mathrm{P}(1) & 115.91(3) & \mathrm{P}(3) & \mathrm{Cu}(1) & \mathrm{P}(1) & 123.67(3)\end{array}$




\begin{tabular}{|c|c|c|c|c|c|c|c|}
\hline $\mathrm{Cu}(1)$ & $P(1)$ & C(31) & 108.2(1) & $\mathrm{Cu}(1)$ & $P(1)$ & $C(37)$ & 116.2(1) \\
\hline $\mathrm{Cu}(1)$ & $P(1)$ & C(43) & $120.1(1)$ & $P(3)$ & $\mathrm{Cu}(1)$ & $P(2)$ & $88.58(3)$ \\
\hline $\mathrm{Cu}(1)$ & $P(2)$ & $C(1)$ & 103.8(1) & $\mathrm{Cu}(1)$ & $P(2)$ & $C(7)$ & 121.5(1) \\
\hline $\mathrm{Cu}(1)$ & $P(2)$ & $C(13)$ & 117.9(1) & $\mathrm{Cu}(1)$ & $P(3)$ & $C(6)$ & $103.8(1)$ \\
\hline $\mathrm{Cu}(1)$ & $P(3)$ & $C(25)$ & 109.5(1) & $\mathrm{Cu}(1)$ & $P(3)$ & C(19) & 129.3(1) \\
\hline$P(5)$ & $\mathrm{Cu}(2)$ & $P(4)$ & $127.49(4)$ & $P(6)$ & $\mathrm{Cu}(2)$ & $P(4)$ & $124.88(4)$ \\
\hline $\mathrm{Cu}(2)$ & $P(4)$ & $C(79)$ & 109.5(1) & $\mathrm{Cu}(2)$ & $P(4)$ & C(85) & 115.5(1) \\
\hline $\mathrm{Cu}(2)$ & $P(4)$ & $\mathrm{C}(91)$ & 119.8(1) & $P(6)$ & $\mathrm{Cu}(2)$ & $P(5)$ & $87.75(3)$ \\
\hline $\mathrm{Cu}(2)$ & $P(5)$ & C(49) & $102.0(1)$ & $\mathrm{Cu}(2)$ & $P(5)$ & $C(61)$ & $113.3(1)$ \\
\hline $\mathrm{Cu}(2)$ & $P(5)$ & C(55) & $127.2(1)$ & $\mathrm{Cu}(2)$ & $P(6)$ & C(54) & 101.7(1) \\
\hline $\mathrm{Cu}(2)$ & $P(6)$ & $C(67)$ & 119.8(1) & $\mathrm{Cu}(2)$ & $P(6)$ & C(73) & $120.4(1)$ \\
\hline$C(37)$ & $P(1)$ & $C(31)$ & $104.8(1)$ & $C(43)$ & $P(1)$ & $C(31)$ & $103.0(1)$ \\
\hline$P(1)$ & $C(31)$ & $C(32)$ & $124.1(3)$ & $P(1)$ & $C(31)$ & $C(36)$ & 118.3(3) \\
\hline$C(43)$ & $P(1)$ & C(37) & $102.8(1)$ & $P(1)$ & C(37) & $C(38)$ & $122.5(3)$ \\
\hline$P(1)$ & $\mathrm{C}(37)$ & $\mathrm{C}(42)$ & 119.8(2) & $P(1)$ & $C(43)$ & $\mathrm{C}(44)$ & 118.6(2) \\
\hline$P(1)$ & $C(43)$ & $C(48)$ & $123.0(3)$ & $P(2)$ & $C(1)$ & $C(6)$ & 118.6(2) \\
\hline $\mathrm{C}(7)$ & $P(2)$ & $C(1)$ & $105.0(1)$ & $C(13)$ & $P(2)$ & $C(1)$ & $102.2(1)$ \\
\hline$P(2)$ & $C(1)$ & $C(2)$ & $121.8(2)$ & $C(13)$ & $P(2)$ & $C(7)$ & 104.1(1) \\
\hline$P(2)$ & C(7) & C(8) & 124.7(2) & $\mathrm{P}(2)$ & $C(7)$ & $C(12)$ & 116.5(2) \\
\hline$P(2)$ & $C(13)$ & $C(14)$ & 123.1(2) & $P(2)$ & $C(13)$ & $C(18)$ & 118.6(2) \\
\hline$P(3)$ & $\mathrm{C}(6)$ & $C(1)$ & $119.0(2)$ & $C(25)$ & $P(3)$ & $C(6)$ & $107.9(2)$ \\
\hline$P(3)$ & $C(6)$ & $C(5)$ & $122.3(2)$ & $C(19)$ & $P(3)$ & $C(6)$ & $102.0(1)$ \\
\hline$C(25)$ & $P(3)$ & $C(19)$ & $102.9(1)$ & $\mathrm{P}(3)$ & $C(19)$ & $C(20)$ & 117.7(2) \\
\hline$P(3)$ & $C(19)$ & $C(24)$ & $123.0(2)$ & $P(3)$ & $C(25)$ & $C(26)$ & 124.6(3) \\
\hline$P(3)$ & $C(25)$ & $C(30)$ & 115.6(3) & $P(4)$ & $C(79)$ & $\mathrm{C}(84)$ & 116.8(2) \\
\hline$C(85)$ & $P(4)$ & $\mathrm{C}(79)$ & 104.6(1) & $\mathrm{C}(91)$ & $P(4)$ & C(79) & 104.4(1) \\
\hline$P(4)$ & C(79) & $C(80)$ & $124.7(2)$ & $P(4)$ & C(85) & $C(90)$ & 118.0(3) \\
\hline$C(91)$ & $P(4)$ & C(85) & 101.6(2) & $P(4)$ & C(85) & $C(86)$ & $123.0(2)$ \\
\hline$P(4)$ & $\mathrm{C}(91)$ & C(92) & $122.8(2)$ & $P(4)$ & $\mathrm{C}(91)$ & $C(96)$ & 119.0(2) \\
\hline$P(5)$ & $C(49)$ & $C(54)$ & 117.7(2) & $C(61)$ & $P(5)$ & C(49) & 102.2(1) \\
\hline$P(5)$ & $C(49)$ & $C(50)$ & 123.0(2) & C(55) & $P(5)$ & $C(49)$ & $105.9(2)$ \\
\hline$C(61)$ & $P(5)$ & C(55) & 103.5(1) & $P(5)$ & C(55) & $C(56)$ & 119.1(2) \\
\hline$P(5)$ & C(55) & $C(60)$ & $122.2(2)$ & $P(5)$ & $C(61)$ & $C(62)$ & 117.2(2) \\
\hline
\end{tabular}

Table 6. Bond angles $\left(^{\circ}\right)$-- continued

\begin{tabular}{|c|c|c|c|c|c|c|c|}
\hline atom & atom & atom & angle & atom & atom & atom & angle \\
\hline$P(5)$ & $C(61)$ & $C(66)$ & $124.1(3)$ & $P(6)$ & C(54) & C(49) & $118.3(2)$ \\
\hline$C(67)$ & $P(6)$ & $C(54)$ & 105.2(1) & $C(73)$ & $P(6)$ & C(54) & 104.2(1) \\
\hline$P(6)$ & $C(54)$ & $C(53)$ & $122.9(2)$ & $C(73)$ & $P(6)$ & $C(67)$ & 103.5(1) \\
\hline$P(6)$ & $C(67)$ & $C(68)$ & $121.2(2)$ & $P(6)$ & C(67) & $C(72)$ & $120.3(2)$ \\
\hline$P(6)$ & $C(73)$ & $C(74)$ & $117.7(2)$ & $P(6)$ & $C(73)$ & $C(78)$ & 124.2(3) \\
\hline$C(6)$ & $C(1)$ & $C(2)$ & 119.5(3) & $C(1)$ & $\mathrm{C}(2)$ & C(3) & 120.9(3) \\
\hline$C(1)$ & $C(6)$ & $C(5)$ & $118.5(3)$ & $C(2)$ & $C(3)$ & $\mathrm{C}(4)$ & 119.4(3) \\
\hline C(3) & $C(4)$ & $C(5)$ & $120.6(3)$ & $C(4)$ & $C(5)$ & $C(6)$ & 121.1(3) \\
\hline$C(12)$ & $C(7)$ & $C(8)$ & 118.7(3) & $C(7)$ & $C(8)$ & $\mathrm{C}(9)$ & 119.9(3) \\
\hline$C(7)$ & $C(12)$ & $C(11)$ & $121.2(3)$ & $\mathrm{C}(8)$ & $\mathrm{C}(9)$ & $C(10)$ & $120.3(3)$ \\
\hline $\mathrm{C}(9)$ & $C(10)$ & $C(11)$ & $120.2(3)$ & $C(10)$ & $C(11)$ & $C(12)$ & 119.6(3) \\
\hline$C(18)$ & $C(13)$ & $C(14)$ & 118.2(3) & $C(13)$ & $C(14)$ & $C(15)$ & 120.8(3) \\
\hline$C(13)$ & $C(18)$ & $C(17)$ & $120.9(3)$ & $C(14)$ & $C(15)$ & $C(16)$ & 120.1(3) \\
\hline$C(15)$ & $C(16)$ & $C(17)$ & 119.8(3) & $C(16)$ & $C(17)$ & $C(18)$ & 120.1(3) \\
\hline
\end{tabular}




$\begin{array}{llllllll}\mathrm{C}(24) & \mathrm{C}(19) & \mathrm{C}(20) & 119.2(3) & \mathrm{C}(19) & \mathrm{C}(20) & \mathrm{C}(21) & 119.5(3) \\ \mathrm{C}(19) & \mathrm{C}(24) & \mathrm{C}(23) & 120.1(3) & \mathrm{C}(20) & \mathrm{C}(21) & \mathrm{C}(22) & 120.9(4) \\ \mathrm{C}(21) & \mathrm{C}(22) & \mathrm{C}(23) & 119.8(3) & \mathrm{C}(22) & \mathrm{C}(23) & \mathrm{C}(24) & 120.5(3) \\ \mathrm{C}(30) & \mathrm{C}(25) & \mathrm{C}(26) & 119.4(3) & \mathrm{C}(25) & \mathrm{C}(26) & \mathrm{C}(27) & 119.2(3) \\ \mathrm{C}(25) & \mathrm{C}(30) & \mathrm{C}(29) & 120.9(3) & \mathrm{C}(26) & \mathrm{C}(27) & \mathrm{C}(28) & 121.3(3) \\ \mathrm{C}(27) & \mathrm{C}(28) & \mathrm{C}(29) & 119.2(3) & \mathrm{C}(28) & \mathrm{C}(29) & \mathrm{C}(30) & 120.1(3) \\ \mathrm{C}(36) & \mathrm{C}(31) & \mathrm{C}(32) & 117.6(3) & \mathrm{C}(31) & \mathrm{C}(32) & \mathrm{C}(33) & 120.2(3) \\ \mathrm{C}(31) & \mathrm{C}(36) & \mathrm{C}(35) & 121.8(4) & \mathrm{C}(32) & \mathrm{C}(33) & \mathrm{C}(34) & 120.5(4) \\ \mathrm{C}(33) & \mathrm{C}(34) & \mathrm{C}(35) & 120.8(4) & \mathrm{C}(34) & \mathrm{C}(35) & \mathrm{C}(36) & 119.1(4) \\ \mathrm{C}(42) & \mathrm{C}(37) & \mathrm{C}(38) & 117.6(3) & \mathrm{C}(37) & \mathrm{C}(38) & \mathrm{C}(39) & 120.6(4) \\ \mathrm{C}(37) & \mathrm{C}(42) & \mathrm{C}(41) & 121.3(3) & \mathrm{C}(38) & \mathrm{C}(39) & \mathrm{C}(40) & 121.3(4) \\ \mathrm{C}(39) & \mathrm{C}(40) & \mathrm{C}(41) & 119.3(4) & \mathrm{C}(40) & \mathrm{C}(41) & \mathrm{C}(42) & 120.0(4) \\ \mathrm{C}(48) & \mathrm{C}(43) & \mathrm{C}(44) & 118.4(3) & \mathrm{C}(43) & \mathrm{C}(44) & \mathrm{C}(45) & 121.2(3) \\ \mathrm{C}(43) & \mathrm{C}(48) & \mathrm{C}(47) & 120.2(3) & \mathrm{C}(44) & \mathrm{C}(45) & \mathrm{C}(46) & 119.8(3) \\ \mathrm{C}(45) & \mathrm{C}(46) & \mathrm{C}(47) & 119.9(3) & \mathrm{C}(46) & \mathrm{C}(47) & \mathrm{C}(48) & 120.6(3) \\ \mathrm{C}(54) & \mathrm{C}(49) & \mathrm{C}(50) & 119.3(3) & \mathrm{C}(49) & \mathrm{C}(50) & \mathrm{C}(51) & 121.0(3) \\ \mathrm{C}(49) & \mathrm{C}(54) & \mathrm{C}(53) & 118.9(3) & \mathrm{C}(50) & \mathrm{C}(51) & \mathrm{C}(52) & 118.9(3) \\ \mathrm{C}(51) & \mathrm{C}(52) & \mathrm{C}(53) & 120.6(3) & \mathrm{C}(52) & \mathrm{C}(53) & \mathrm{C}(54) & 121.3(3) \\ \mathrm{C}(60) & \mathrm{C}(55) & \mathrm{C}(56) & 118.6(3) & \mathrm{C}(55) & \mathrm{C}(56) & \mathrm{C}(57) & 121.7(3) \\ \mathrm{C}(55) & \mathrm{C}(60) & \mathrm{C}(59) & 120.1(3) & \mathrm{C}(56) & \mathrm{C}(57) & \mathrm{C}(58) & 119.1(3) \\ \mathrm{C}(57) & \mathrm{C}(58) & \mathrm{C}(59) & 120.3(3) & \mathrm{C}(58) & \mathrm{C}(59) & \mathrm{C}(60) & 120.2(3) \\ \mathrm{C}(66) & \mathrm{C}(61) & \mathrm{C}(62) & 118.3(3) & \mathrm{C}(61) & \mathrm{C}(62) & \mathrm{C}(63) & 121.3(3) \\ \mathrm{C}(61) & \mathrm{C}(66) & \mathrm{C}(65) & 120.4(3) & \mathrm{C}(62) & \mathrm{C}(63) & \mathrm{C}(64) & 119.4(3) \\ & & & & & & & \end{array}$

Table 6. Bond angles $\left(^{\circ}\right)$-- continued

$\begin{array}{llllllll}\text { atom } & \text { atom } & \text { atom } & \text { angle } & \text { atom } & \text { atom } & \text { atom } & \text { angle } \\ \mathrm{C}(63) & \mathrm{C}(64) & \mathrm{C}(65) & 120.6(3) & \mathrm{C}(64) & \mathrm{C}(65) & \mathrm{C}(66) & 119.9(3) \\ \mathrm{C}(72) & \mathrm{C}(67) & \mathrm{C}(68) & 118.0(3) & \mathrm{C}(67) & \mathrm{C}(68) & \mathrm{C}(69) & 120.6(3) \\ \mathrm{C}(67) & \mathrm{C}(72) & \mathrm{C}(71) & 120.6(3) & \mathrm{C}(68) & \mathrm{C}(69) & \mathrm{C}(70) & 120.8(3) \\ \mathrm{C}(69) & \mathrm{C}(70) & \mathrm{C}(71) & 119.5(3) & \mathrm{C}(70) & \mathrm{C}(71) & \mathrm{C}(72) & 120.4(3) \\ \mathrm{C}(78) & \mathrm{C}(73) & \mathrm{C}(74) & 118.0(3) & \mathrm{C}(73) & \mathrm{C}(74) & \mathrm{C}(75) & 120.6(3) \\ \mathrm{C}(73) & \mathrm{C}(78) & \mathrm{C}(77) & 120.9(3) & \mathrm{C}(74) & \mathrm{C}(75) & \mathrm{C}(76) & 119.9(3) \\ \mathrm{C}(75) & \mathrm{C}(76) & \mathrm{C}(77) & 119.8(3) & \mathrm{C}(76) & \mathrm{C}(77) & \mathrm{C}(78) & 120.7(3) \\ \mathrm{C}(84) & \mathrm{C}(79) & \mathrm{C}(80) & 118.3(3) & \mathrm{C}(79) & \mathrm{C}(80) & \mathrm{C}(81) & 120.7(3) \\ \mathrm{C}(79) & \mathrm{C}(84) & \mathrm{C}(83) & 121.0(3) & \mathrm{C}(80) & \mathrm{C}(81) & \mathrm{C}(82) & 120.5(3) \\ \mathrm{C}(81) & \mathrm{C}(82) & \mathrm{C}(83) & 119.9(3) & \mathrm{C}(82) & \mathrm{C}(83) & \mathrm{C}(84) & 119.5(3) \\ \mathrm{C}(90) & \mathrm{C}(85) & \mathrm{C}(86) & 118.9(3) & \mathrm{C}(85) & \mathrm{C}(86) & \mathrm{C}(87) & 120.8(3) \\ \mathrm{C}(85) & \mathrm{C}(90) & \mathrm{C}(89) & 120.0(3) & \mathrm{C}(86) & \mathrm{C}(87) & \mathrm{C}(88) & 119.8(4) \\ \mathrm{C}(87) & \mathrm{C}(88) & \mathrm{C}(89) & 120.7(3) & \mathrm{C}(88) & \mathrm{C}(89) & \mathrm{C}(90) & 119.8(3) \\ \mathrm{C}(96) & \mathrm{C}(91) & \mathrm{C}(92) & 118.2(3) & \mathrm{C}(91) & \mathrm{C}(92) & \mathrm{C}(93) & 121.4(3) \\ \mathrm{C}(91) & \mathrm{C}(96) & \mathrm{C}(95) & 120.0(3) & \mathrm{C}(92) & \mathrm{C}(93) & \mathrm{C}(94) & 120.5(4) \\ \mathrm{C}(93) & \mathrm{C}(94) & \mathrm{C}(95) & 119.3(4) & \mathrm{C}(94) & \mathrm{C}(95) & \mathrm{C}(96) & 120.6(3)\end{array}$

Table 8. Torsion Angles $\left({ }^{\circ}\right)$

$\begin{array}{lllllllllr}\text { atom1 } & \text { atom2 } & \text { atom3 } & \text { atom4 } & \text { angle } & \text { atom1 } & \text { atom2 } & \text { atom3 } & \text { atom4 } & \text { angle } \\ \mathrm{I}(1) & \mathrm{Cu}(1) & \mathrm{P}(1) & \mathrm{C}(31) & -43.0(1) & \mathrm{I}(1) & \mathrm{Cu}(1) & \mathrm{P}(1) & \mathrm{C}(37) & 74.5(1) \\ \mathrm{I}(1) & \mathrm{Cu}(1) & \mathrm{P}(1) & \mathrm{C}(43) & -160.7(1) & \mathrm{P}(2) & \mathrm{Cu}(1) & \mathrm{P}(1) & \mathrm{C}(31) & 84.1(1) \\ \mathrm{P}(2) & \mathrm{Cu}(1) & \mathrm{P}(1) & \mathrm{C}(37) & -158.4(1) & \mathrm{P}(2) & \mathrm{Cu}(1) & \mathrm{P}(1) & \mathrm{C}(43) & -33.6(1)\end{array}$




\begin{tabular}{|c|c|c|c|c|c|c|c|c|c|}
\hline$P(3)$ & $\mathrm{Cu}(1)$ & $P(1)$ & $C(31)$ & $-169.1(1)$ & $P(3)$ & $\mathrm{Cu}(1)$ & $P(1)$ & $C(37)$ & $-51.6(1)$ \\
\hline$P(3)$ & $\mathrm{Cu}(1)$ & $P(1)$ & $C(43)$ & $73.2(1)$ & $\mathrm{I}(1)$ & $\mathrm{Cu}(1)$ & $P(2)$ & $C(1)$ & $-91.6(1)$ \\
\hline I(1) & $\mathrm{Cu}(1)$ & $P(2)$ & $C(7)$ & $26.0(1)$ & $\mathrm{I}(1)$ & $\mathrm{Cu}(1)$ & $P(2)$ & $C(13)$ & 156.3(1) \\
\hline$P(1)$ & $\mathrm{Cu}(1)$ & $P(2)$ & $C(1)$ & 149.10(9) & $P(1)$ & $\mathrm{Cu}(1)$ & $P(2)$ & $C(7)^{\prime}$ & $-93.4(1)$ \\
\hline$P(1)$ & $\mathrm{Cu}(1)$ & $P(2)$ & $C(13)$ & $37.0(1)$ & $P(3)$ & $\mathrm{Cu}(1)$ & $P(2)$ & $C(1)$ & $21.92(9)$ \\
\hline$P(3)$ & $\mathrm{Cu}(1)$ & $P(2)$ & $C(7)$ & $139.5(1)$ & $P(3)$ & $\mathrm{Cu}(1)$ & $P(2)$ & $C(13)$ & $-90.2(1)$ \\
\hline I(1) & $\mathrm{Cu}(1)$ & $P(3)$ & $C(6)$ & $98.6(1)$ & $\mathrm{I}(1)$ & $\mathrm{Cu}(1)$ & $P(3)$ & $C(19)$ & $-19.9(1)$ \\
\hline I(1) & $\mathrm{Cu}(1)$ & $P(3)$ & $C(25)$ & $-146.4(1)$ & $P(1)$ & $\mathrm{Cu}(1)$ & $P(3)$ & $C(6)^{\prime}$ & $-139.8(1)$ \\
\hline$P(1)$ & $\mathrm{Cu}(1)$ & $P(3)$ & $C(19)$ & $101.7(1)$ & $P(1)$ & $\mathrm{Cu}(1)$ & $P(3)$ & $C(25)$ & $-24.8(1)$ \\
\hline$P(2)$ & $\mathrm{Cu}(1)$ & $P(3)$ & $C(6)$ & $-19.3(1)$ & $P(2)$ & $\mathrm{Cu}(1)$ & $P(3)$ & $C(19)$ & $-137.8(1)$ \\
\hline$P(2)$ & $\mathrm{Cu}(1)$ & $P(3)$ & $C(25)$ & $95.7(1)$ & $\mathrm{I}(2)$ & $\mathrm{Cu}(2)$ & $P(4)$ & $C(79)$ & $-30.6(1)$ \\
\hline$I(2)$ & $\mathrm{Cu}(2)$ & $P(4)$ & $C(85)$ & 87.1(1) & I(2) & $\mathrm{Cu}(2)$ & $P(4)$ & $C(91)$ & $-151.0(1)$ \\
\hline$P(5)$ & $\mathrm{Cu}(2)$ & $P(4)$ & $C(79)$ & $90.3(1)$ & $P(5)$ & $\mathrm{Cu}(2)$ & $P(4)$ & $C(85)$ & $-152.0(1)$ \\
\hline$P(5)$ & $\mathrm{Cu}(2)$ & $P(4)$ & $C(91)$ & $-30.1(1)$ & $P(6)$ & $\mathrm{Cu}(2)$ & $P(4)$ & $C(79)$ & $-151.4(1)$ \\
\hline$P(6)$ & $\mathrm{Cu}(2)$ & $P(4)$ & $C(85)$ & $-33.7(1)$ & $P(6)$ & $\mathrm{Cu}(2)$ & $P(4)$ & $C(91)$ & $88.2(1)$ \\
\hline$I(2)$ & $\mathrm{Cu}(2)$ & $P(5)$ & $C(49)$ & $-68.9(1)$ & $I(2)$ & $\mathrm{Cu}(2)$ & $P(5)$ & $C(55)$ & $51.9(1)$ \\
\hline $\mathrm{I}(2)$ & $\mathrm{Cu}(2)$ & $P(5)$ & $C(61)$ & $-177.9(1)$ & $P(4)$ & $\mathrm{Cu}(2)$ & $P(5)$ & $C(49)$ & $164.81(9)$ \\
\hline$P(4)$ & $\mathrm{Cu}(2)$ & $P(5)$ & $C(55)$ & $-74.4(1)$ & $P(4)$ & $\mathrm{Cu}(2)$ & $P(5)$ & $C(61)$ & $55.8(1)$ \\
\hline$P(6)$ & $\mathrm{Cu}(2)$ & $P(5)$ & $C(49)$ & 31.1(1) & $P(6)$ & $\mathrm{Cu}(2)$ & $P(5)$ & $C(55)$ & $151.9(1)$ \\
\hline$P(6)$ & $\mathrm{Cu}(2)$ & $P(5)$ & $C(61)$ & $-77.9(1)$ & $\mathrm{I}(2)$ & $\mathrm{Cu}(2)$ & $P(6)$ & $C(54)$ & $70.1(1)$ \\
\hline $\mathrm{I}(2)$ & $\mathrm{Cu}(2)$ & $P(6)$ & $C(67)$ & $-45.2(1)$ & $\mathrm{I}(2)$ & $\mathrm{Cu}(2)$ & $P(6)$ & $C(73)$ & $-175.6(1)$ \\
\hline$P(4)$ & $\mathrm{Cu}(2)$ & $P(6)$ & $C(54)$ & $-164.06(9)$ & $P(4)$ & $\mathrm{Cu}(2)$ & $P(6)$ & $C(67)$ & $80.7(1)$ \\
\hline$P(4)$ & $\mathrm{Cu}(2)$ & $P(6)$ & $C(73)$ & $-49.8(1)$ & $P(5)$ & $\mathrm{Cu}(2)$ & $P(6)$ & $C(54)$ & $-28.4(1)$ \\
\hline$P(5)$ & $\mathrm{Cu}(2)$ & $P(6)$ & $C(67)$ & $-143.7(1)$ & $P(5)$ & $\mathrm{Cu}(2)$ & $P(6)$ & $C(73)$ & $85.9(1)$ \\
\hline $\mathrm{Cu}(1)$ & $P(1)$ & $C(31)$ & $C(32)$ & $122.8(3)$ & $\mathrm{Cu}(1)$ & $P(1)$ & $C(31)$ & $C(36)$ & $-55.1(3)$ \\
\hline C(37) & $P(1)$ & $C(31)$ & $C(32)$ & $-1.8(3)$ & $C(37)$ & $P(1)$ & $C(31)$ & $C(36)$ & $-179.7(2)$ \\
\hline$C(43)$ & $P(1)$ & $C(31)$ & $C(32)$ & $-109.0(3)$ & $C(43)$ & $P(1)$ & $C(31)$ & $C(36)$ & $73.1(3)$ \\
\hline $\mathrm{Cu}(1)$ & $P(1)$ & $C(37)$ & $C(38)$ & $147.7(3)$ & $\mathrm{Cu}(1)$ & $P(1)$ & $C(37)$ & $C(42)$ & $-29.2(3)$ \\
\hline$C(31)$ & $P(1)$ & $C(37)$ & $C(38)$ & $-93.0(3)$ & $C(31)$ & $P(1)$ & $C(37)$ & $C(42)$ & $90.1(3)$ \\
\hline$C(43)$ & $P(1)$ & $C(37)$ & $C(38)$ & $14.4(3)$ & $C(43)$ & $P(1)$ & $C(37)$ & $C(42)$ & $-162.5(3)$ \\
\hline $\mathrm{Cu}(1)$ & $P(1)$ & $C(43)$ & C(44) & $-49.0(3)$ & $\mathrm{Cu}(1)$ & $P(1)$ & $C(43)$ & $C(48)$ & $133.1(3)$ \\
\hline$C(31)$ & $P(1)$ & $C(43)$ & $C(44)$ & $-169.3(2)$ & $C(31)$ & $P(1)$ & $C(43)$ & $C(48)$ & $12.7(3)$ \\
\hline$C(37)$ & $P(1)$ & $C(43)$ & $C(44)$ & $82.0(3)$ & $C(37)$ & $P(1)$ & $C(43)$ & $C(48)$ & $-95.9(3)$ \\
\hline $\mathrm{Cu}(1)$ & $P(2)$ & $C(1)$ & $C(2)$ & $161.7(2)$ & $\mathrm{Cu}(1)$ & $P(2)$ & $C(1)$ & $C(6)$ & $-21.3(2)$ \\
\hline
\end{tabular}

Table 8. Torsion angles $\left(^{0}\right)$-- continued

$\begin{array}{llllllllll}\text { atom1 } & \text { atom2 } & \text { atom3 } & \text { atom4 } & \text { angle } & \text { atom1 } & \text { atom2 } & \text { atom3 } & \text { atom4 } & \text { angle } \\ \mathrm{C}(7) & \mathrm{P}(2) & \mathrm{C}(1) & \mathrm{C}(2) & 33.1(3) & \mathrm{C}(7) & \mathrm{P}(2) & \mathrm{C}(1) & \mathrm{C}(6) & -149.8(2) \\ \mathrm{C}(13) & \mathrm{P}(2) & \mathrm{C}(1) & \mathrm{C}(2) & -75.2(2) & \mathrm{C}(13) & \mathrm{P}(2) & \mathrm{C}(1) & \mathrm{C}(6) & 101.8(2) \\ \mathrm{Cu}(1) & \mathrm{P}(2) & \mathrm{C}(7) & \mathrm{C}(8) & 150.2(2) & \mathrm{Cu}(1) & \mathrm{P}(2) & \mathrm{C}(7) & \mathrm{C}(12) & -27.5(3) \\ \mathrm{C}(1) & \mathrm{P}(2) & \mathrm{C}(7) & \mathrm{C}(8) & -92.9(3) & \mathrm{C}(1) & \mathrm{P}(2) & \mathrm{C}(7) & \mathrm{C}(12) & 89.5(3) \\ \mathrm{C}(13) & \mathrm{P}(2) & \mathrm{C}(7) & \mathrm{C}(8) & 14.2(3) & \mathrm{C}(13) & \mathrm{P}(2) & \mathrm{C}(7) & \mathrm{C}(12) & -163.5(2) \\ \mathrm{Cu}(1) & \mathrm{P}(2) & \mathrm{C}(13) & \mathrm{C}(14) & 105.4(3) & \mathrm{Cu}(1) & \mathrm{P}(2) & \mathrm{C}(13) & \mathrm{C}(18) & -72.3(3) \\ \mathrm{C}(1) & \mathrm{P}(2) & \mathrm{C}(13) & \mathrm{C}(14) & -7.6(3) & \mathrm{C}(1) & \mathrm{P}(2) & \mathrm{C}(13) & \mathrm{C}(18) & 174.8(2) \\ \mathrm{C}(7) & \mathrm{P}(2) & \mathrm{C}(13) & \mathrm{C}(14) & -116.6(3) & \mathrm{C}(7) & \mathrm{P}(2) & \mathrm{C}(13) & \mathrm{C}(18) & 65.7(3) \\ \mathrm{Cu}(1) & \mathrm{P}(3) & \mathrm{C}(6) & \mathrm{C}(1) & 12.1(2) & \mathrm{Cu}(1) & \mathrm{P}(3) & \mathrm{C}(6) & \mathrm{C}(5) & -162.3(2) \\ \mathrm{C}(19) & \mathrm{P}(3) & \mathrm{C}(6) & \mathrm{C}(1) & 148.1(2) & \mathrm{C}(19) & \mathrm{P}(3) & \mathrm{C}(6) & \mathrm{C}(5) & -26.3(3) \\ \mathrm{C}(25) & \mathrm{P}(3) & \mathrm{C}(6) & \mathrm{C}(1) & -104.0(2) & \mathrm{C}(25) & \mathrm{P}(3) & \mathrm{C}(6) & \mathrm{C}(5) & 81.6(3) \\ \mathrm{Cu}(1) & \mathrm{P}(3) & \mathrm{C}(19) & \mathrm{C}(20) & 36.9(3) & \mathrm{Cu}(1) & \mathrm{P}(3) & \mathrm{C}(19) & \mathrm{C}(24) & -147.5(3) \\ \mathrm{C}(6) & \mathrm{P}(3) & \mathrm{C}(19) & \mathrm{C}(20) & -82.3(3) & \mathrm{C}(6) & \mathrm{P}(3) & \mathrm{C}(19) & \mathrm{C}(24) & 93.2(3)\end{array}$




\begin{tabular}{|c|c|c|c|c|c|c|c|c|c|}
\hline$C(25)$ & $P(3)$ & $C(19)$ & $C(20)$ & $165.9(3)$ & $C(25)$ & $P(3)$ & $C(19)$ & $C(24)$ & $-18.5(3)$ \\
\hline $\mathrm{Cu}(1)$ & $P(3)$ & $C(25)$ & $C(26)$ & $-103.9(3)$ & $\mathrm{Cu}(1)$ & $P(3)$ & $C(25)$ & $C(30)$ & $68.5(2)$ \\
\hline$C(6)$ & $P(3)$ & $C(25)$ & $C(26)$ & $8.5(3)$ & $C(6)$ & $P(3)$ & $C(25)$ & $C(30)$ & $-179.2(2)$ \\
\hline C(19) & $P(3)$ & $C(25)$ & $C(26)$ & $115.8(3)$ & C(19) & $P(3)$ & $C(25)$ & $C(30)$ & $-71.9(2)$ \\
\hline $\mathrm{Cu}(2)$ & $P(4)$ & $\mathrm{C}(79)$ & $C(80)$ & $130.4(3)$ & $\mathrm{Cu}(2)$ & $P(4)$ & $C(79)$ & $C(84)$ & $-45.0(3)$ \\
\hline$C(85)$ & $P(4)$ & $C(79)$ & $C(80)$ & $6.0(3)$ & $C(85)$ & $P(4)$ & $C(79)$ & $C(84)$ & $-169.3(2)$ \\
\hline C(91) & $P(4)$ & $C(79)$ & $\mathrm{C}(80)$ & $-100.3(3)$ & $C(91)$ & $P(4)$ & $C(79)$ & $C(84)$ & $84.4(3)$ \\
\hline $\mathrm{Cu}(2)$ & $P(4)$ & $C(85)$ & $C(86)$ & $166.9(2)$ & $\mathrm{Cu}(2)$ & $P(4)$ & $C(85)$ & $C(90)$ & $-17.0(3)$ \\
\hline$C(79)$ & $P(4)$ & $C(85)$ & $C(86)$ & $-72.7(3)$ & $C(79)$ & $P(4)$ & $C(85)$ & $C(90)$ & $103.4(2)$ \\
\hline$C(91)$ & $P(4)$ & $C(85)$ & $C(86)$ & $35.7(3)$ & $C(91)$ & $P(4)$ & $C(85)$ & $C(90)$ & $-148.2(2)$ \\
\hline $\mathrm{Cu}(2)$ & $P(4)$ & $C(91)$ & $C(92)$ & 101.6(3) & $\mathrm{Cu}(2)$ & $P(4)$ & $C(91)$ & $C(96)$ & $-75.9(3)$ \\
\hline C(79) & $P(4)$ & $C(91)$ & $C(92)$ & $-21.3(3)$ & $C(79)$ & $P(4)$ & $C(91)$ & $C(96)$ & $161.2(3)$ \\
\hline C(85) & $P(4)$ & $C(91)$ & $C(92)$ & $-129.9(3)$ & $C(85)$ & $P(4)$ & $C(91)$ & $C(96)$ & $52.6(3)$ \\
\hline $\mathrm{Cu}(2)$ & $P(5)$ & $C(49)$ & $C(50)$ & $153.0(2)$ & $\mathrm{Cu}(2)$ & $P(5)$ & $C(49)$ & $C(54)$ & $-28.4(2)$ \\
\hline$C(55)$ & $P(5)$ & $C(49)$ & $C(50)$ & $18.4(3)$ & $C(55)$ & $P(5)$ & C(49) & $C(54)$ & $-163.1(2)$ \\
\hline$C(61)$ & $P(5)$ & $C(49)$ & $C(50)$ & $-89.6(2)$ & $C(61)$ & $P(5)$ & $C(49)$ & $C(54)$ & $88.9(2)$ \\
\hline $\mathrm{Cu}(2)$ & $P(5)$ & $C(55)$ & $C(56)$ & $-16.5(3)$ & $\mathrm{Cu}(2)$ & $P(5)$ & $C(55)$ & $C(60)$ & $160.6(2)$ \\
\hline C(49) & $P(5)$ & $C(55)$ & $C(56)$ & 102.6(3) & $C(49)$ & $P(5)$ & $C(55)$ & $C(60)$ & $-80.3(3)$ \\
\hline C(61) & $P(5)$ & $C(55)$ & $C(56)$ & $-150.3(3)$ & $C(61)$ & $P(5)$ & $C(55)$ & $C(60)$ & $26.7(3)$ \\
\hline $\mathrm{Cu}(2)$ & $P(5)$ & $C(61)$ & $C(62)$ & $-57.0(3)$ & $\mathrm{Cu}(2)$ & $P(5)$ & $C(61)$ & $C(66)$ & $116.0(3)$ \\
\hline C(49) & $P(5)$ & $C(61)$ & $C(62)$ & $-165.9(2)$ & $C(49)$ & $P(5)$ & $C(61)$ & $C(66)$ & $7.1(3)$ \\
\hline C(55) & $P(5)$ & $C(61)$ & $C(62)$ & $84.3(2)$ & $C(55)$ & $P(5)$ & $C(61)$ & $C(66)$ & $-102.7(3)$ \\
\hline $\mathrm{Cu}(2)$ & $P(6)$ & $C(54)$ & $C(49)$ & $19.4(2)$ & $\mathrm{Cu}(2)$ & $P(6)$ & $C(54)$ & $C(53)$ & $-160.2(2)$ \\
\hline C(67) & $P(6)$ & $C(54)$ & C(49) & $145.0(2)$ & $C(67)$ & $P(6)$ & $C(54)$ & $C(53)$ & $-34.6(3)$ \\
\hline
\end{tabular}

Table 8. Torsion angles $\left({ }^{0}\right)$-- continued

\begin{tabular}{|c|c|c|c|c|c|c|c|c|c|}
\hline atom1 & atom2 & atom3 & atom4 & angle & atom1 & atom2 & atom3 & atom4 & angle \\
\hline $\mathrm{C}(73)$ & $P(6)$ & $C(54)$ & $C(49)$ & $-106.4(2)$ & $C(73)$ & $P(6)$ & $C(54)$ & $C(53)$ & $74.0(3)$ \\
\hline $\mathrm{Cu}(2)$ & $P(6)$ & $C(67)$ & $C(68)$ & $-98.9(3)$ & $\mathrm{Cu}(2)$ & $P(6)$ & $C(67)$ & $C(72)$ & 72.5(3) \\
\hline$C(54)$ & $P(6)$ & $C(67)$ & $C(68)$ & $147.7(2)$ & $C(54)$ & $P(6)$ & $C(67)$ & $C(72)$ & $-40.8(3)$ \\
\hline C(73) & $P(6)$ & $C(67)$ & $C(68)$ & $38.6(3)$ & C(73) & $P(6)$ & $C(67)$ & $C(72)$ & $-149.9(2)$ \\
\hline $\mathrm{Cu}(2)$ & $P(6)$ & $C(73)$ & $C(74)$ & $-6.3(3)$ & $\mathrm{Cu}(2)$ & $P(6)$ & $C(73)$ & $\mathrm{C}(78)$ & $174.3(3)$ \\
\hline$C(54)$ & $P(6)$ & $C(73)$ & $C(74)$ & $106.6(3)$ & $C(54)$ & $P(6)$ & $C(73)$ & $C(78)$ & $-72.8(3)$ \\
\hline$C(67)$ & $P(6)$ & $C(73)$ & $C(74)$ & $-143.5(3)$ & $C(67)$ & $P(6)$ & $C(73)$ & $C(78)$ & $37.1(3)$ \\
\hline$P(2)$ & $C(1)$ & $C(2)$ & $C(3)$ & $177.8(3)$ & $C(6)$ & $C(1)$ & $C(2)$ & $C(3)$ & $0.8(4)$ \\
\hline$P(2)$ & $C(1)$ & $C(6)$ & $P(3)$ & $6.3(3)$ & $P(2)$ & $C(1)$ & $C(6)$ & $C(5)$ & $-179.1(3)$ \\
\hline$C(2)$ & $C(1)$ & $C(6)$ & $P(3)$ & $-176.6(2)$ & $C(2)$ & $C(1)$ & $C(6)$ & $C(5)$ & $-2.0(4)$ \\
\hline$C(1)$ & $C(2)$ & $C(3)$ & $C(4)$ & $0.2(4)$ & $C(2)$ & $C(3)$ & $C(4)$ & $C(5)$ & $-0.0(5)$ \\
\hline$C(3)$ & $C(4)$ & C(5) & $C(6)$ & $-1.3(5)$ & $\mathrm{C}(4)$ & $C(5)$ & $C(6)$ & $P(3)$ & $176.7(2)$ \\
\hline$C(4)$ & $C(5)$ & $C(6)$ & $C(1)$ & $2.3(5)$ & $P(2)$ & $C(7)$ & $C(8)$ & $C(9)$ & $-176.2(3)$ \\
\hline$C(12)$ & $C(7)$ & $C(8)$ & C(9) & $1.5(5)$ & $P(2)$ & $C(7)$ & $C(12)$ & $C(11)$ & 177.0(3) \\
\hline$C(8)$ & $C(7)$ & $C(12)$ & $C(11)$ & $-0.8(5)$ & $C(7)$ & $C(8)$ & $C(9)$ & $C(10)$ & $-0.4(5)$ \\
\hline $\mathrm{C}(8)$ & $\mathrm{C}(9)$ & $C(10)$ & $C(11)$ & $-1.4(5)$ & $C(9)$ & $C(10)$ & $C(11)$ & $C(12)$ & $2.0(5)$ \\
\hline$C(10)$ & $C(11)$ & $C(12)$ & $C(7)$ & $-0.9(5)$ & $P(2)$ & $C(13)$ & $C(14)$ & $C(15)$ & $-176.8(3)$ \\
\hline$C(18)$ & $C(13)$ & $C(14)$ & $C(15)$ & $0.9(5)$ & $\mathrm{P}(2)$ & $C(13)$ & $C(18)$ & $C(17)$ & $178.4(3)$ \\
\hline$C(14)$ & $C(13)$ & $C(18)$ & $C(17)$ & $0.6(5)$ & $C(13)$ & $C(14)$ & $C(15)$ & $C(16)$ & $-2.2(5)$ \\
\hline$C(14)$ & $C(15)$ & $C(16)$ & $C(17)$ & $1.8(5)$ & $C(15)$ & $C(16)$ & $C(17)$ & $C(18)$ & $-0.3(5)$ \\
\hline$C(16)$ & $C(17)$ & $C(18)$ & $C(13)$ & $-0.9(5)$ & $P(3)$ & $C(19)$ & $C(20)$ & $C(21)$ & $177.5(3)$ \\
\hline$C(24)$ & $C(19)$ & $C(20)$ & $C(21)$ & $1.8(6)$ & $P(3)$ & $C(19)$ & $C(24)$ & $C(23)$ & $-178.3(3)$ \\
\hline$C(20)$ & $C(19)$ & $C(24)$ & $C(23)$ & $-2.8(5)$ & $C(19)$ & $C(20)$ & $C(21)$ & $C(22)$ & $-0.2(5)$ \\
\hline
\end{tabular}




$\begin{array}{lllll}\mathrm{C}(20) & \mathrm{C}(21) & \mathrm{C}(22) & \mathrm{C}(23) & -0.5(6) \\ \mathrm{C}(22) & \mathrm{C}(23) & \mathrm{C}(24) & \mathrm{C}(19) & 2.1(6) \\ \mathrm{C}(30) & \mathrm{C}(25) & \mathrm{C}(26) & \mathrm{C}(27) & 0.2(4) \\ \mathrm{C}(26) & \mathrm{C}(25) & \mathrm{C}(30) & \mathrm{C}(29) & -1.0(5) \\ \mathrm{C}(26) & \mathrm{C}(27) & \mathrm{C}(28) & \mathrm{C}(29) & -0.1(4) \\ \mathrm{C}(28) & \mathrm{C}(29) & \mathrm{C}(30) & \mathrm{C}(25) & 1.3(5) \\ \mathrm{C}(36) & \mathrm{C}(31) & \mathrm{C}(32) & \mathrm{C}(33) & -2.8(5) \\ \mathrm{C}(32) & \mathrm{C}(31) & \mathrm{C}(36) & \mathrm{C}(35) & 4.3(5) \\ \mathrm{C}(32) & \mathrm{C}(33) & \mathrm{C}(34) & \mathrm{C}(35) & 1.7(6) \\ \mathrm{C}(34) & \mathrm{C}(35) & \mathrm{C}(36) & \mathrm{C}(31) & -2.8(6) \\ \mathrm{C}(42) & \mathrm{C}(37) & \mathrm{C}(38) & \mathrm{C}(39) & 0.5(6) \\ \mathrm{C}(38) & \mathrm{C}(37) & \mathrm{C}(42) & \mathrm{C}(41) & 0.8(5) \\ \mathrm{C}(38) & \mathrm{C}(39) & \mathrm{C}(40) & \mathrm{C}(41) & -0.2(5) \\ \mathrm{C}(40) & \mathrm{C}(41) & \mathrm{C}(42) & \mathrm{C}(37) & -1.8(6)\end{array}$

Table 8. Torsion angles $\left(^{\circ}\right)$-- continued

$\begin{array}{lllll}\mathrm{C}(21) & \mathrm{C}(22) & \mathrm{C}(23) & \mathrm{C}(24) & -0.5(6) \\ \mathrm{P}(3) & \mathrm{C}(25) & \mathrm{C}(26) & \mathrm{C}(27) & 172.2(3) \\ \mathrm{P}(3) & \mathrm{C}(25) & \mathrm{C}(30) & \mathrm{C}(29) & -173.8(3) \\ \mathrm{C}(25) & \mathrm{C}(26) & \mathrm{C}(27) & \mathrm{C}(28) & 0.4(5) \\ \mathrm{C}(27) & \mathrm{C}(28) & \mathrm{C}(29) & \mathrm{C}(30) & -0.7(5) \\ \mathrm{P}(1) & \mathrm{C}(31) & \mathrm{C}(32) & \mathrm{C}(33) & 179.4(3) \\ \mathrm{P}(1) & \mathrm{C}(31) & \mathrm{C}(36) & \mathrm{C}(35) & -177.7(3) \\ \mathrm{C}(31) & \mathrm{C}(32) & \mathrm{C}(33) & \mathrm{C}(34) & -0.1(4) \\ \mathrm{C}(33) & \mathrm{C}(34) & \mathrm{C}(35) & \mathrm{C}(36) & -0.3(6) \\ \mathrm{P}(1) & \mathrm{C}(37) & \mathrm{C}(38) & \mathrm{C}(39) & -176.5(3) \\ \mathrm{P}(1) & \mathrm{C}(37) & \mathrm{C}(42) & \mathrm{C}(41) & 177.9(3) \\ \mathrm{C}(37) & \mathrm{C}(38) & \mathrm{C}(39) & \mathrm{C}(40) & -0.8(7) \\ \mathrm{C}(39) & \mathrm{C}(40) & \mathrm{C}(41) & \mathrm{C}(42) & 1.5(6) \\ \mathrm{P}(1) & \mathrm{C}(43) & \mathrm{C}(44) & \mathrm{C}(45) & -177.1(3)\end{array}$




$\begin{array}{llllllllll}\mathrm{P}(4) & \mathrm{C}(91) & \mathrm{C}(92) & \mathrm{C}(93) & -178.0(3) & \mathrm{C}(96) & \mathrm{C}(91) & \mathrm{C}(92) & \mathrm{C}(93) & -0.4(6) \\ \mathrm{P}(4) & \mathrm{C}(91) & \mathrm{C}(96) & \mathrm{C}(95) & 177.4(3) & \mathrm{C}(92) & \mathrm{C}(91) & \mathrm{C}(96) & \mathrm{C}(95) & -0.2(5) \\ \mathrm{C}(91) & \mathrm{C}(92) & \mathrm{C}(93) & \mathrm{C}(94) & 1.2(6) & \mathrm{C}(92) & \mathrm{C}(93) & \mathrm{C}(94) & \mathrm{C}(95) & -1.3(7) \\ \mathrm{C}(93) & \mathrm{C}(94) & \mathrm{C}(95) & \mathrm{C}(96) & 0.7(6) & \mathrm{C}(94) & \mathrm{C}(95) & \mathrm{C}(96) & \mathrm{C}(91) & 0.1(5)\end{array}$

The sign is positive if when looking from atom 2 to atom 3 a clock-wise motion of atom 1 would superimpose it on atom 4 . 